\title{
RIBBON BRAIDED MODULE CATEGORIES, QUANTUM SYMMETRIC PAIRS AND KNIZHNIK-ZAMOLODCHIKOV EQUATIONS
}

\author{
KENNY DE COMMER, SERGEY NESHVEYEV, LARS TUSET, AND MAKOTO YAMASHITA
}

\begin{abstract}
Let $\mathfrak{u}$ be a compact semisimple Lie algebra, and $\sigma$ be a Lie algebra involution of $\mathfrak{u}$. Let $\operatorname{Rep}_{q}(\mathfrak{u})$ be the ribbon braided tensor $\mathrm{C}^{*}$-category of admissible $U_{q}(\mathfrak{u})$-representations for $0<q<1$. We introduce three module $C^{*}$-categories over $\operatorname{Rep}_{q}(\mathfrak{u})$ starting from the input data $(\mathfrak{u}, \sigma)$. The first construction is based on the theory of 2-cyclotomic KZ-equations. The second construction uses the notion of quantum symmetric pair as developed by G. Letzter. The third construction uses a variation of Drinfeld twisting. In all three cases the module $\mathrm{C}^{*}$-category is ribbon twist-braided in the sense of A. Brochier - this is essentially due to B. Enriquez in the first case, is proved by S. Kolb in the second case, and is closely related to work of J. Donin, P. Kulish, and A. Mudrov in the third case. We formulate a conjecture concerning equivalence of these ribbon twist-braided module $\mathrm{C}^{*}$-categories, and confirm it in the rank one case.
\end{abstract}

\section{INTRODUCTION}

The Tannaka-Krein principle for quantum groups asserts that there is a duality between Hopf algebras on the one hand, and concretely represented tensor categories with duals on the other. Here, we say that a tensor category $\mathcal{C}$ is concretely represented if there is a fiber functor realizing $\mathcal{C}$ within the category of vector spaces. From this viewpoint, a tensor category is more fundamental than a Hopf algebra, as the latter can be seen as a concrete manifestation of the former through a fiber functor, while an abstract tensor category can occur in more general contexts, for instance, as a hidden symmetry in quantum field theories.

This viewpoint is beautifully illustrated by the result of V. Drinfeld [Dri89] which in the framework of braided tensor categories provides two apparently unrelated constructions starting from a semisimple Lie algebra $\mathfrak{g}$. The first construction is algebraic, where the category is the representation category $\operatorname{Rep}_{q}(\mathfrak{g})$ of $U_{q}(\mathfrak{g})$, the $q$-deformed universal enveloping algebra of $\mathfrak{g}$, viewed as a quasi-triangular Hopf algebra. The second construction is analytic, and builds a new associator on the category of $\mathfrak{g}$-representations by means of the monodromy of the Knizhnik-Zamolodchikov equations arising from conformal field theory. In both approaches, the crucial point is that one should look for a system which contains a braiding obtained by deforming the symmetric braiding appearing in the classical Tannaka-Krĕn duality theorem.

If quantum groups correspond to tensor categories, their actions correspond to module categories. Such structures are more elaborate, partly reflected through new phenomena such as the distinction between 'homogeneous spaces' and 'subgroups' already present in the case $\mathfrak{g}=\mathfrak{s l}_{2}(\mathbb{C})$, see $\operatorname{Pod} 87$. Although a full classification seems difficult, there is a very useful ansatz producing module categories from the theory of reflection equations, which stems from the study of quantum scattering on the half-line by I. Cherednik Che84.

While there are many interesting developments in the relation between the reflection equation and quantum group actions ( KSS93 GS99, DM02 DKM03, Mud07, KS09] to list a few), the main motivation for the present work is the interplay between the theory of KZ-equation of type B on the analytic side, and quantum symmetric pairs for semisimple Lie algebras on the algebraic side.

Let $\mathfrak{g}$ be a complex semisimple Lie algebra, $\theta$ an involution of $\mathfrak{g}$ with spectral decomposition $\mathfrak{g}=\mathfrak{g}^{\theta} \oplus \mathfrak{p}$, where $\mathfrak{g}^{\theta}$ is the fixed point Lie algebra. Choose a Cartan subalgebra $\mathfrak{h}$ of $\mathfrak{g}$ which is stable under $\theta$ and such that $\mathfrak{h} \cap \mathfrak{p}$ is a maximal ad $_{\mathfrak{g}}$-diagonalizable abelian subspace of $\mathfrak{p}$. G. Letzter Let99.Let00 realizes a quantization of $\mathfrak{g}^{\theta}$ as a coideal subalgebra of $U_{q}(\mathfrak{g})$ built using a root system of $(\mathfrak{g}, \mathfrak{h})$, depending possibly on some extra parameters. The categorical counterpart is given by the representation category of this coideal subalgebra as a module category over $\operatorname{Rep}_{q}(\mathfrak{g})$. Recent works of M. Balagović and S. Kolb BK15b Kol17, making use of the formalism of the universal $K$-matrix DKM03, show that this module

Date: December 19, 2017; Minor revisions February 13, 2018; September 12, 2018.

The work of K. De Commer was partially supported by the FWO grant G.0251.15N and the grant H2020-MSCA-RISE2015-691246-QUANTUM DYNAMICS. The work of S. Neshveyev was partially supported by the ERC grant 307663NCGQG. 
category is a ribbon twist-braided module category in the sense of A. Brochier Bro13. Note that the theory of (universal) $K$-matrices forms the concrete connection with the theory of reflection equations.

On the other hand, the theory of 2-cyclotomic KZ-equations (or, KZ-equations of type B) Che90, Lei94, GL00, Enr07 makes it possible to construct, for an arbitrary involution $\sigma$ of $\mathfrak{g}$, an action of $\operatorname{Rep}(\mathfrak{g})$ with the KZ-associator on $\operatorname{Rep}\left(\mathfrak{g}^{\sigma}\right)$ and a non-trivial mixed associator. Based on the results of [Enr07] and Bro12], one observes that this is actually again a ribbon twist-braided module category.

The goal of this paper is to perform the above constructions in the presence of a $\mathrm{C}^{*}$-structure, corresponding to the compact form $\mathfrak{u}$ of $\mathfrak{g}$, and to state a precise conjecture concerning the equivalence of the resulting ribbon twist-braided module $\mathrm{C}^{*}$-categories in case $\sigma=\theta$. We also present a third picture, conjecturally equivalent to the above two, where we consider involutions $\nu$ of $\mathfrak{u}$ for which the prescribed Cartan subalgebra $\mathfrak{t} \subseteq \mathfrak{u}$ is stable and $\mathfrak{t}^{\nu}$ is a Cartan subalgebra of $\mathfrak{u}^{\nu}$. In this situation, the involution quantizes straightforwardly to an automorphism of $U_{q}(\mathfrak{u})$. Using a $\nu$-twisted version of the Drinfeld double construction, we construct a $*$-algebra with coaction by $U_{q}(\mathfrak{u})$ such that its category of admissible *-representations becomes a ribbon twist-braided module $\mathrm{C}^{*}$-category over $\operatorname{Rep}_{q}(\mathfrak{u})$. Here the construction of the braiding on the module $\mathrm{C}^{*}$-category is a variation on the construction of a universal $K$-matrix in DKM03.

The precise structure of the paper is as follows.

In Section 1 we recall the formalism of ribbon twist-braided module categories [Bro13, Kol17 in the setting of non-trivial associators and non-trivial autoequivalence of the acting tensor category. In Section 2 we present an overview of the main elements of the theory of symmetric pairs for compact semisimple Lie algebras that we will make use of. In Section 3 we present in detail the three constructions of twist-braided module $\mathrm{C}^{*}$-categories associated to compact symmetric pairs that were mentioned above. We then state our main conjecture regarding the equivalence of these ribbon twist-braided module $\mathrm{C}^{*}$ categories in Section 4. We verify the conjecture in Section 5 for the rank one case.

In the Appendix we treat in more detail the following topics. In the first part we discuss the two extremal positions for a stable Cartan subalgebra with respect to an involution on a semisimple compact Lie algebra. In the second part we investigate compatibility of the $*$-structure of $U_{q}(\mathfrak{u})$ with the coideal symmetric pairs of Let99, complementing the discussion in Let02 concerning twisted $*$-invariance. In the third part we provide a link between the choice of parameters for a coideal symmetric pair, and the character theory of the associated non-parameter coideal symmetric pair. In the fourth part we discuss a relation between ribbon braids and cylinder twists.

Acknowledgements: We thank Weiqiang Wang for bringing our attention to [BW18], and the anonymous reviewer for careful reading and useful comments to improve readability.

Conventions. We assume in the following, unless stated otherwise, that all categories are $\mathbb{C}$-linear and semisimple - in particular morphism spaces are finite dimensional. We also assume that all (bi)functors are $\mathbb{C}$-(bi)linear. We identify identity morphisms with their objects, $\operatorname{id}_{X}=X$. For $\sigma$ an endomorphism on a real vector space $V$, we will denote by the same symbol its extension to a complex-linear endomorphism of $V^{\mathbb{C}}=V \otimes_{\mathbb{R}} \mathbb{C}$.

We use the following notation for $q$-integers, $q$-factorials, and $q$-binomial coefficients:

$$
[n]_{q}=\frac{q^{-n}-q^{n}}{q^{-1}-q}, \quad[n]_{q} !=[n]_{q}[n-1]_{q} \cdots[2]_{q}[1]_{q}, \quad\left[\begin{array}{c}
m \\
n
\end{array}\right]_{q}=\frac{[m]_{q} !}{[n]_{q} ![m-n]_{q} !}
$$

\section{Ribbon twist-BRaided module C*-CAtegories}

We mainly follow the conventions of NT13 for tensor $\mathrm{C}^{*}$-categories. Additional basic material on module categories can be found in, e.g., [Müg10,EGNO15].

Definition 1.1. A tensor category is given by:

- a category $\mathcal{C}$,

- a bifunctor $\otimes: \mathcal{C} \times \mathcal{C} \rightarrow \mathcal{C}$,

- a distinguished object $\mathbb{1}$ of $\mathcal{C}$,

- a natural isomorphism $\Phi_{U, V, W}:(U \otimes V) \otimes W \rightarrow U \otimes(V \otimes W)$, and

- natural isomorphisms $\lambda_{U}: \mathbb{1} \otimes U \rightarrow U, \rho_{U}: U \otimes \mathbb{1} \rightarrow U$ 
satisfying a standard set of axioms. We often use the symbol $\mathcal{C}$ to denote the above set of data. A tensor $C^{*}$-category is a tensor category whose underlying category is a $\mathrm{C}^{*}$-category and for which $\otimes$ is a $*$-bifunctor and $\Phi, \lambda$, and $\rho$ are unitary natural transformations.

Definition 1.2. Let $\mathcal{C}$ be a tensor category as above. A (right) module category over $\mathcal{C}$ is given by:

- a category $\mathcal{D}$,

- a bifunctor $\odot: \mathcal{D} \times \mathcal{C} \rightarrow \mathcal{D}$, and

- natural isomorphisms $r_{X}: X \odot \mathbb{1} \rightarrow X$ and

$$
\Psi_{X, U, V}:(X \odot U) \odot V \rightarrow X \odot(U \otimes V)
$$

for which the following diagrams commute (unit relations and the mixed pentagon relation):
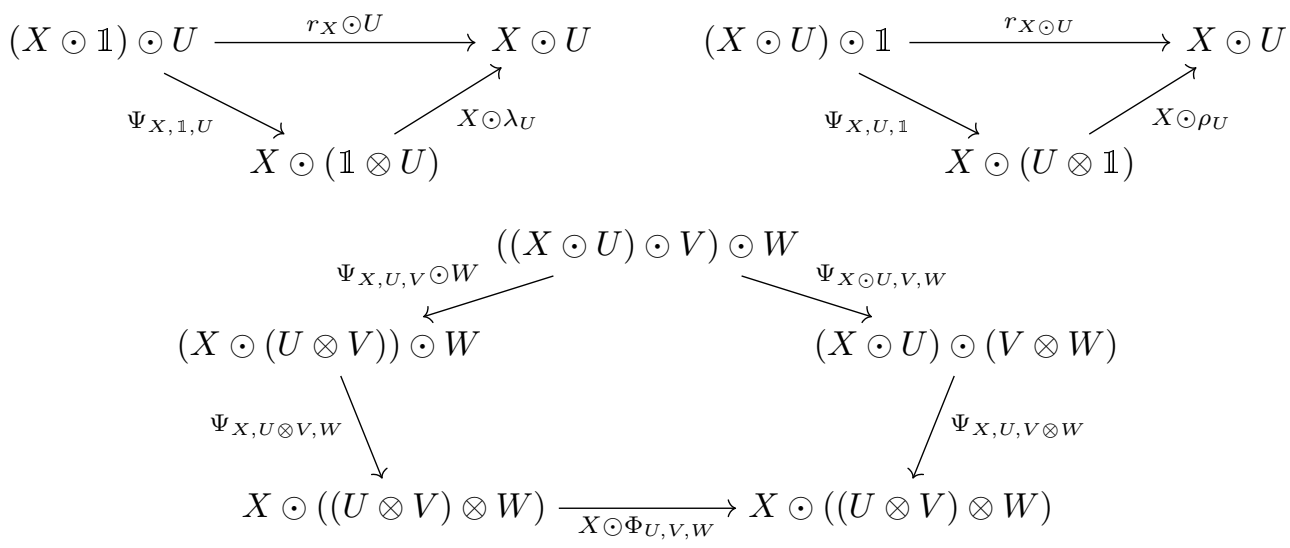

Again, we often denote the whole structure by $\mathcal{D}$.

When $\mathcal{C}$ is a tensor $\mathrm{C}^{*}$-category, we call $\mathcal{D}$ a module $\mathrm{C}^{*}$-category if $\mathcal{D}$ is a $\mathrm{C}^{*}$-category, $\odot$ is a $*$ bifunctor, and $\Psi, r$ are unitary.

In the following we will assume that the unit $\mathbb{1}$ of $\mathcal{C}$ is strict, and that it acts as a strict unit on $\mathcal{D}$, so $X \odot \mathbb{1}=X$ and $r_{X}=X$. This will be satisfied in all our examples.

Definition 1.3. A braided tensor category is a tensor category $\mathcal{C}$ endowed with natural isomorphisms $\beta_{U, V}: U \otimes V \rightarrow V \otimes U$ satisfying the hexagon equation

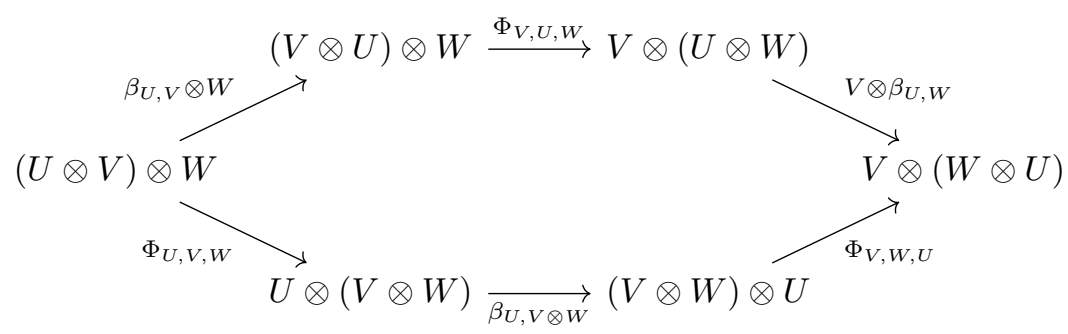

and the similar relation with $\beta_{U, V}$ replaced by $\beta_{V, U}^{-1}$. We also assume $\beta_{\mathbb{1}, U}=\beta_{U, \mathbb{1}}=U$.

A monoidal autoequivalence of $\mathcal{C}$ consists of an equivalence $\sigma: \mathcal{C} \rightarrow \mathcal{C}$ and natural isomorphisms

$$
\left(\sigma_{2}\right)_{U, V}: \sigma(U) \otimes \sigma(V) \rightarrow \sigma(U \otimes V)
$$

satisfying natural compatibility conditions with respect to the associator $\Phi$. A braided autoequivalence of $\mathcal{C}$ is a monoidal autoequivalence $\sigma$ for which the following diagram commutes:

$$
\begin{array}{cc}
\sigma(U) \otimes \sigma(V) \stackrel{\beta_{\sigma(U), \sigma(V)}}{\longrightarrow} \sigma(V) \otimes \sigma(U) & \\
\left(\sigma_{2}\right)_{U, V} \downarrow & \\
\sigma(U \otimes V) \stackrel{{ }^{2}\left(\sigma_{2}\right)_{V, U}}{\longrightarrow} & \sigma(V \otimes U)
\end{array}
$$

The following definition was introduced in [Bro13, Section 5], see also Kol17, Remark 3.15]. However, we use a slightly different convention than the latter reference in the presence of a non-trivial braided autoequivalence (apart from introducing explicitly the necessary associators in the non-strict setting). 
Definition 1.4. Let $\mathcal{C}$ be a braided tensor category, and $\sigma: \mathcal{C} \rightarrow \mathcal{C}$ a braided monoidal autoequivalence. A right module category $\mathcal{D}$ over $\mathcal{C}$ is said to be $\sigma$-braided when $\mathcal{D}$ is equipped with a natural isomorphism $\eta_{X, U}: X \odot \sigma(U) \rightarrow X \odot U$, called the $\sigma$-braid of $\mathcal{D}$, which satisfies the $\sigma$-octagon equation

$$
\begin{aligned}
& (X \odot U) \odot \sigma(V) \stackrel{\Psi_{X, U, \sigma(V)}}{\longrightarrow} X \odot(U \otimes \sigma(V)) \stackrel{X \odot \beta_{U, \sigma(V)}}{\longrightarrow} X \odot(\sigma(V) \otimes U) \stackrel{\Psi_{X, \sigma(V), U}^{-1}}{\longrightarrow}(X \odot \sigma(V)) \odot U \\
& \eta_{X \odot U, V} \downarrow \\
& (X \odot U) \odot V \underset{\Psi_{X, U, V}^{-1}}{\longleftarrow} X \odot(U \otimes V) \underset{X \odot \beta_{V, U}}{\longleftarrow} X \odot(V \otimes U) \stackrel{\eta_{X, V} \odot U}{\Psi_{X, V, U}}(X \odot V) \odot U
\end{aligned}
$$

We say that $\eta$ is a ribbon $\sigma$-braid if, moreover, the ribbon $\sigma$-twist equation is satisfied:

$$
\begin{aligned}
& X \odot \sigma(U \otimes V) \stackrel{X \odot\left(\sigma_{2}\right)_{U, V}^{-1}}{\longrightarrow} X \odot(\sigma(U) \otimes \sigma(V)) \stackrel{\Psi_{X, \sigma(U), \sigma(V)}^{-1}}{\longrightarrow}(X \odot \sigma(U)) \odot \sigma(V) \\
& \eta_{X, U \otimes V} \downarrow \quad \downarrow \downarrow \eta_{X, U} \odot \sigma(V) \\
& X \odot(U \otimes V) \longleftarrow \Psi_{X, U, V}(X \odot U) \odot V \longleftarrow \eta_{X \odot U, V}(X \odot U) \odot \sigma(V)
\end{aligned}
$$

We say that $\mathcal{D}$ is (ribbon) twist-braided if $\sigma$ is implicit, and that $\mathcal{D}$ is (ribbon) braided if $\sigma$ is the identity.

Remarks 1.5. (1) If all our structures including $\sigma$ are strict, our definition will give a (ribbon) $\sigma^{-1}$-braid in the sense of Kol17, Remark 3.15], taking $e_{M, V}=\eta_{M, \sigma^{-1}(V)}$.

(2) In the $\mathrm{C}^{*}$-setting, besides the condition of module $\mathrm{C}^{*}$-category, we just assume that $\sigma$ is a $*$ autoequivalence and $\mathcal{D}$ is a $\mathcal{C}$-module $\mathrm{C}^{*}$-category. We do not assume that $\beta$ and $\eta$ are unitary, as this would be too restrictive.

The following definition clarifies the notion of equivalence we will use between different (ribbon) twist-braided module $\mathrm{C}^{*}$-categories.

Definition 1.6. Let $\mathcal{C}$ be a braided tensor category with braided monoidal autoequivalences $\sigma$ and $\sigma^{\prime}$, and let $(\mathcal{D}, \odot, \Psi, \eta)$, resp. $\left(\mathcal{D}^{\prime}, \odot^{\prime}, \Psi^{\prime}, \eta^{\prime}\right)$ be a $\sigma$-braided, resp. $\sigma^{\prime}$-braided module category over $\mathcal{C}$. We say that $\mathcal{D}$ and $\mathcal{D}^{\prime}$ are equivalent as twist-braided module categories, written $\mathcal{D} \cong \mathcal{D}^{\prime}$, if there exist an isomorphism of monoidal functors $F: \sigma^{\prime} \rightarrow \sigma$, and an equivalence $G: \mathcal{D} \rightarrow \mathcal{D}^{\prime}$ of categories together with natural isomorphisms

such that the diagrams

$$
\left(G_{2}\right)_{X, U}: G(X) \odot^{\prime} U \rightarrow G(X \odot U)
$$

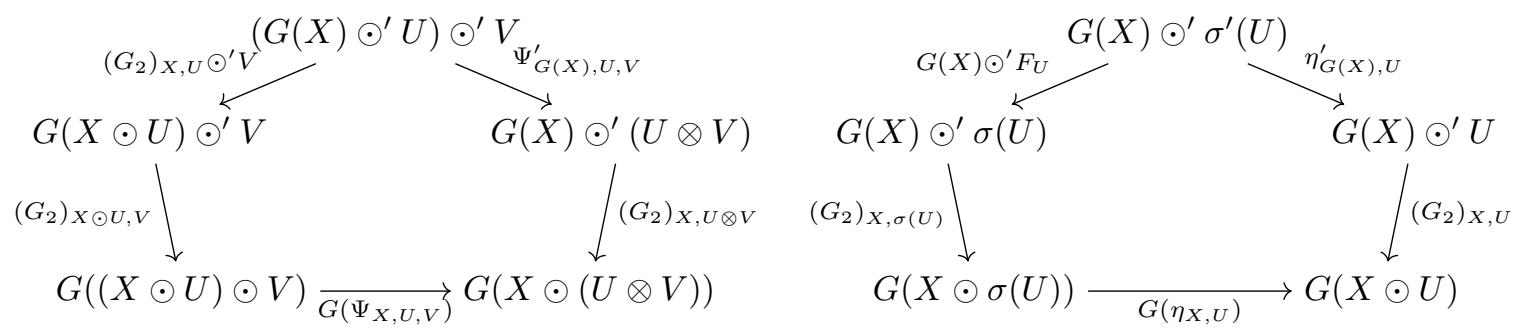

commute. In the $\mathrm{C}^{*}$-setting we ask that $G$ is a $*$-functor, and that $F$ and $G_{2}$ are unitary.

Note that $F$ is uniquely determined if it exists.

For completeness, let us briefly recall the graphical presentation of (ribbon) twist-braids, following Bro13. We represent composition of morphisms by vertical stacking, $\xi \eta$ being represented by the part for $\xi$ on top of that for $\eta$. The monoidal product is represented by horizontal juxtaposition. As usual, we represent the braiding of $\mathcal{C}$ by the tangle 1.a. A twist-braid can be represented by the diagram 1.b, so that the twist octagon relation can be presented as 1.c (suppressing the module category associator $\Psi)$.

On the other hand, ribbon twist-braids need to be represented by 2.a, so that regular isotopy of strands in 2.b represents the ribbon twist equation.

Remark 1.7. The extra twist can be gotten rid of by slightly changing the axiomatics of the ribbon twist-braids. This change of axiomatics does not affect anything as long as the acting tensor category has a ribbon twist, see [BZBJ16, Remark 3.6]. 


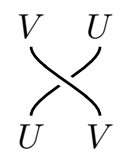

(a) braid

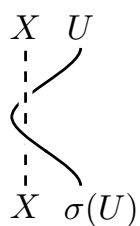

(b) twist-braid

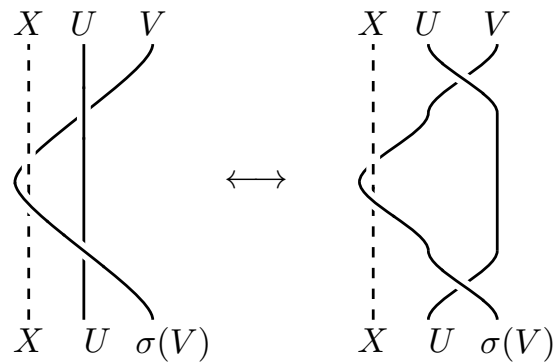

(c) twist octagon relation

FiguRE 1. twist-braids

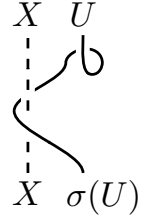

(a) ribbon twist-braid

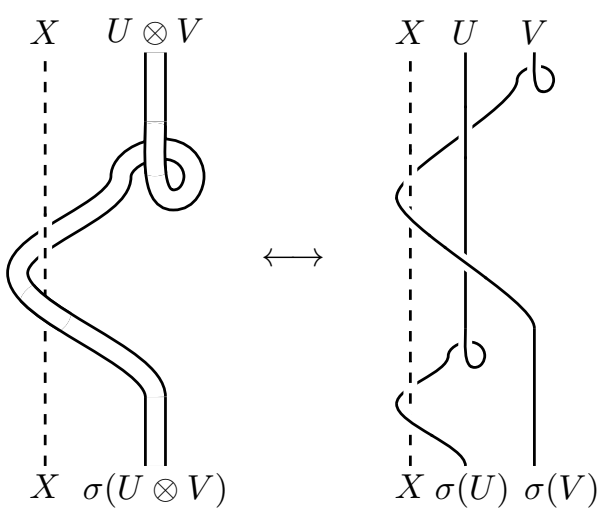

(b) ribbon twist equation

FIGURE 2. ribbon twist-braids

One can obtain module categories from algebraic structures in the following way. We momentarily neglect the semisimplicity condition on our categories.

Definition 1.8 (Dri89, Kas95, Chapter XV]). A (unital and counital) quasi-bialgebra is given by

- a unital algebra $A$,

- unital algebra homomorphisms $\Delta: A \rightarrow A \otimes A$ and $\varepsilon: A \rightarrow \mathbb{C}$, and

- an invertible element $\phi \in A \otimes A \otimes A$

satisfying

$$
\begin{gathered}
(\mathrm{id} \otimes \Delta) \Delta(a)=\phi((\Delta \otimes \mathrm{id}) \Delta(a)) \phi^{-1}, \quad(\mathrm{id} \otimes \mathrm{id} \otimes \Delta)(\phi)(\Delta \otimes \mathrm{id} \otimes \mathrm{id})(\phi)=\phi_{234}(\mathrm{id} \otimes \Delta \otimes \mathrm{id})(\phi) \phi_{123}, \\
(\varepsilon \otimes \mathrm{id}) \Delta(a)=a=(\mathrm{id} \otimes \varepsilon) \Delta(a), \quad(\mathrm{id} \otimes \varepsilon \otimes \mathrm{id})(\phi)=1 \otimes 1 .
\end{gathered}
$$

From the above conditions we also have $(\mathrm{id} \otimes \mathrm{id} \otimes \varepsilon)(\phi)=1=(\varepsilon \otimes \mathrm{id} \otimes \mathrm{id})(\phi)$. The category $A$-Mod of left $A$-modules becomes a tensor category as follows. When $U, V, W$ are $A$-modules, $U \otimes V$ is the usual tensor product over $\mathbb{C}$ with the left $A$-module structure $a \cdot(u \otimes v)=\Delta(a)(u \otimes v)$, and the associator is given by

$$
\Phi_{U, V, W}(u \otimes v \otimes w)=\phi(u \otimes v \otimes w) .
$$

In this context the rigidity of $A$-Mod can be encoded by a generalization of antipode, and quasi-bialgebras with such antipodes are called quasi-Hopf algebras.

Definition 1.9. Let $A$ be a quasi-bialgebra, and $B$ a unital algebra. A (normalized right) quasi-coaction of $A$ on $B$ is given by a unital homomorphism $\alpha: B \rightarrow B \otimes A$ and an invertible element $\psi \in B \otimes A \otimes A$ satisfying

$$
\begin{gathered}
(\mathrm{id} \otimes \varepsilon \otimes \mathrm{id})(\psi)=(\mathrm{id} \otimes \mathrm{id} \otimes \varepsilon)(\psi)=1 \otimes 1, \\
(1 \otimes \phi)(\mathrm{id} \otimes \Delta \otimes \mathrm{id})(\psi)(\psi \otimes 1)=(\mathrm{id} \otimes \mathrm{id} \otimes \Delta)(\psi)(\alpha \otimes \mathrm{id} \otimes \mathrm{id})(\psi), \\
(\mathrm{id} \otimes \Delta) \alpha(b)=\psi(\alpha \otimes \mathrm{id}) \alpha(b) \psi^{-1}, \quad(\mathrm{id} \otimes \varepsilon) \alpha(b)=b .
\end{gathered}
$$


The following lemma is immediate.

Lemma 1.10. In the above setting, B-Mod becomes an (A-Mod)-module category as follows. When $X$ is a $B$-module and $U, V$ are $A$-modules, $X \otimes U$ is a $B$-module by $b \cdot(x \otimes u)=\alpha(b)(x \otimes u)$, and the associator is given by

$$
\Psi_{X, U, V}(x \otimes v \otimes w)=\psi(x \otimes u \otimes v) .
$$

It is also easy to see that this way we get a one-to-one correspondence between module category structures on $B$-Mod with strict unit conditions and quasi-coactions on $B$.

Additional braided structure can be implemented analogously. Recall that a quasi-triangular quasibialgebra is given by a quasi-bialgebra $A$ together with an invertible element $\mathscr{R} \in A \otimes A$ satisfying

$$
\mathscr{R} \Delta(a) \mathscr{R}^{-1}=\Delta(a)_{21}, \quad(\Delta \otimes \mathrm{id})(\mathscr{R})=\phi_{312} \mathscr{R}_{13} \phi_{132}^{-1} \mathscr{R}_{23} \phi, \quad(\mathrm{id} \otimes \Delta)(\mathscr{R})=\phi_{231}^{-1} \mathscr{R}_{13} \phi_{213} \mathscr{R}_{12} \phi^{-1} .
$$

Then $A$-Mod becomes a braided tensor category with

$$
\beta_{U, V}(u \otimes v)=\Sigma \mathscr{R}(u \otimes v),
$$

where $\Sigma: U \otimes V \rightarrow V \otimes U$ is the flip map $u \otimes v \mapsto v \otimes u$. Let $\sigma$ be an automorphism of $(A, \Delta, \phi, \mathscr{R})$, so in particular

$$
(\sigma \otimes \sigma \otimes \sigma)(\phi)=\phi, \quad(\sigma \otimes \sigma)(\mathscr{R})=\mathscr{R} .
$$

It then defines a (strict) braided autoequivalence of $A$-Mod, which we denote by the same symbol $\sigma$ : if $U$ is a left $A$-module, then $\sigma(U)=U$ as a vector space, while the module structure is given by $a \cdot{ }_{\sigma} x=\sigma(a) x$.

Definition 1.11. Let $(B, \alpha, \psi)$ be a quasi-coaction for a quasi-triangular quasi-bialgebra $(A, \Delta, \phi, \mathscr{R})$. A $\sigma$-braid for $B$ is an invertible element $\mathscr{E} \in B \otimes A$ such that $\mathscr{E}(\mathrm{id} \otimes \sigma) \alpha(b)=\alpha(b) \mathscr{E}$ for all $b \in B$ and $\mathscr{E}$ satisfies the $\sigma$-octagon relation

$$
(\alpha \otimes \mathrm{id})(\mathscr{E})=\psi^{-1} \mathscr{R}_{21} \psi_{021} \mathscr{E}_{02}(\mathrm{id} \otimes \mathrm{id} \otimes \sigma)\left(\psi_{021}^{-1} \mathscr{R}_{12} \psi\right) .
$$

We say that $\mathscr{E}$ is a ribbon $\sigma$-braid if moreover the ribbon $\sigma$-braid equation holds:

$$
(\mathrm{id} \otimes \Delta)(\mathscr{E})=\mathscr{R}_{21} \psi_{021} \mathscr{E}_{02}(\mathrm{id} \otimes \mathrm{id} \otimes \sigma)\left(\psi_{021}^{-1} \mathscr{R}_{12} \psi\right) \mathscr{E}_{01}(\mathrm{id} \otimes \sigma \otimes \sigma)\left(\psi^{-1}\right) .
$$

Note that in the above equations $B$ is considered at place 0 .

The following lemma is now immediate.

Lemma 1.12. Let $\mathscr{E}$ be a $\sigma$-braid (resp. ribbon $\sigma$-braid) for $(B, \alpha, \psi)$. Then $B$-Mod becomes a $\sigma$-braided (resp. ribbon $\sigma$-braided) A-Mod-module category by

$$
\eta_{X, U}(x \otimes u)=\mathscr{E}(x \otimes u) .
$$

Remark 1.13. Assume that $(B, \alpha, \psi)$ is a quasi-coaction for a quasi-triangular quasi-bialgebra $(A, \Delta, \phi, R)$, $\mathscr{E}$ is a (ribbon) $\sigma$-braid and $g \in A$ is a group-like element, that is, $g$ is an invertible element satisfying $\Delta(g)=g \otimes g$. Then $(\operatorname{Ad} g) \sigma: a \mapsto g \sigma(a) g^{-1}$ is an automorphism of $(A, \Delta, \phi, \mathscr{R})$ again and $\mathscr{E}\left(1 \otimes g^{-1}\right)$ is a (ribbon) (Ad $g) \sigma$-braid for $(B, \alpha, \psi)$, and the associated twist-braided module categories are equivalent. Therefore, if $\sigma$ is inner and implemented by a group-like element, we can pass from the $\sigma$-braided setting to the usual one. It follows that by replacing $A$ by the crossed product $A \rtimes_{\sigma} \mathbb{Z}$ we can always get (ribbon) braids from (ribbon) $\sigma$-braids. Note also that if $\sigma$ is of finite order $n$, then instead of $\mathbb{Z}$ we can take $\mathbb{Z} / n \mathbb{Z}$.

In the setting we will be interested in, the elements $\phi, \psi, \mathscr{R}, \mathscr{E}$ will live in a completed tensor product. More precisely, assume that we have a dense unital $\Delta$-preserving embedding $A \subseteq M(\mathscr{A})$, where $\mathscr{A}=$ $(\mathscr{A}, \Delta)$ is a discrete multiplier quasi-bialgebra [NT11a], so as an algebra

$$
\mathscr{A} \cong \bigoplus_{\lambda \in \Lambda} \operatorname{End}\left(V_{\lambda}\right)
$$

for some index set $\Lambda$ and finite-dimensional vector spaces $\left(V_{\lambda}\right)_{\lambda \in \Lambda}$. Here $M(\mathscr{A})$ is the multiplier algebra of the (non-unital) algebra $\mathscr{A}$, which we can identify with

$$
M(\mathscr{A}) \cong \prod_{\lambda \in \Lambda} \operatorname{End}\left(V_{\lambda}\right)
$$

The embedding of $A \subseteq M(\mathscr{A})$ is dense in the sense that $x A y=x \mathscr{A} y$ for all elements $x, y$ in $\mathscr{A}$. For $B$ a unital algebra, we write

$$
B \hat{\otimes} A \hat{\otimes} \ldots \hat{\otimes} A=M(B \otimes \mathscr{A} \otimes \ldots \otimes \mathscr{A})=\prod_{\lambda, \ldots, \mu} B \otimes \operatorname{End}\left(V_{\lambda} \otimes \ldots \otimes V_{\mu}\right),
$$


and we make use of the natural inclusions of the form

$$
A \otimes \cdots \otimes A \subseteq A \hat{\otimes} \cdots \hat{\otimes} A .
$$

We then call generalized quasi-coaction of $A$ on $B$ any homomorphism $\alpha: B \rightarrow B \hat{\otimes} A$ together with an invertible element $\psi \in B \hat{\otimes} A \hat{\otimes} A$ satisfying

$$
\psi(\alpha \otimes \mathrm{id}) \alpha(b) \psi^{-1}=(\mathrm{id} \otimes \Delta) \alpha(b),
$$

interpreting the identity in $B \hat{\otimes} A \hat{\otimes} A$. Similarly the notion of (ribbon) $\sigma$-braid can be modified by only requiring $\mathscr{E} \in B \hat{\otimes} A$.

\section{Symmetric PAIRS OF Lie ALGEBRAS AND SYMmetriC SPACES}

In this section we recall some elements of the theory of symmetric pairs and symmetric spaces.

2.1. Symmetric pairs and symmetric spaces. By a Lie *-algebra we mean a complex Lie algebra $\mathfrak{g}$ endowed with an involutive conjugate-linear anti-homomorphism $*: \mathfrak{g} \rightarrow \mathfrak{g}$. Let $\mathfrak{g}_{0}$ be a real Lie algebra with complexification $\mathfrak{g}=\mathfrak{g}_{0}^{\mathbb{C}}=\mathfrak{g}_{0} \otimes_{\mathbb{R}} \mathbb{C}$. We can recover $\mathfrak{g}_{0}$ from $\mathfrak{g}$ as $\mathfrak{g}_{0}=\left\{x \in \mathfrak{g} \mid x^{*}=-x\right\}$ if we endow $\mathfrak{g}$ with the Lie $*$-algebra structure

$$
*: \mathfrak{g} \rightarrow \mathfrak{g}, \quad x+i y \mapsto(x+i y)^{*}=-x+i y, \quad\left(x, y \in \mathfrak{g}_{0}\right) .
$$

Every Lie $*$-algebra $(\mathfrak{g}, *)$ is obtained from a real Lie algebra $\mathfrak{g}_{0}$ as above, and we call $\mathfrak{g}_{0} \subseteq \mathfrak{g}$ the associated real form of $(\mathfrak{g}, *)$. In particular, when $\mathfrak{g}$ is semisimple with the Killing form $B(x, y)=\operatorname{Tr}\left(\operatorname{ad}_{\mathfrak{g}}(x) \operatorname{ad}_{\mathfrak{g}}(y)\right)$, we reserve the notation $*$ for the $*$-structure defined by a fixed compact real form $\mathfrak{u} \subseteq \mathfrak{g}$. Note that the associated sesqui-linear form $\langle x, y\rangle=B\left(x, y^{*}\right)$ on $\mathfrak{g}$ will be positive definite.

In the following we fix a compact semisimple Lie algebra $\mathfrak{u}$.

Definition 2.1. Let $\mathfrak{k} \subsetneq \mathfrak{u}$ be a proper real Lie subalgebra. The pair $\mathfrak{k} \subseteq \mathfrak{u}$ is called a compact symmetric pair if there exists an involutive Lie algebra automorphism $\sigma$ of $\mathfrak{u}$ such that $\mathfrak{k}=\mathfrak{u}^{\sigma}$, the fixed point Lie algebra of $\sigma$.

Note that the nontrivial involutive automorphisms of $\mathfrak{u}$ are in one-to-one correspondence with the *-preserving Lie algebra involutions ${ }^{1}$ of $\mathfrak{g}=\mathfrak{u}^{\mathbb{C}}$. Since the Killing form is $\sigma$-invariant, $\sigma$ is unitary with respect to the Hermitian scalar product on $\mathfrak{g}$, and it follows that $\sigma$ is uniquely determined by $\mathfrak{k}$.

Let $U$ be a simply connected compact Lie group (unique up to isomorphism) integrating $\mathfrak{u}$. Let $\sigma$ be an involution on $\mathfrak{u}$. Then $\sigma$ integrates uniquely to an involutive Lie group automorphism of $U$, again denoted by $\sigma$. Let $K=U^{\sigma}$ be the compact Lie subgroup of $\sigma$-fixed points. We have that $K$ is connected Hel01, Theorem VII.8.2], with Lie algebra $\mathfrak{k}$.

Definition 2.2. With $K \subseteq U$ as above, we call $U / K$ the standard symmetric space associated to $\sigma$.

We will in the following always consider $U / K$ as a $U$-space by the natural action $U \curvearrowright U / K$. By isomorphisms of symmetric spaces we mean $U$-equivariant homeomorphisms.

Definition 2.3. Two involutions $\sigma_{1}, \sigma_{2}$ of $\mathfrak{u}$, or the corresponding symmetric pairs, are called equivalent if there exists an automorphism of $\mathfrak{u}$ carrying $\mathfrak{k}_{1}=\mathfrak{u}^{\sigma_{1}}$ to $\mathfrak{k}_{2}=\mathfrak{u}^{\sigma_{2}}$. We call $\sigma_{1}$ and $\sigma_{2}$ inner equivalent if we can choose the automorphism of the form $\operatorname{Ad}(u)$ for some $u \in U$.

It is clear that $\sigma_{1}, \sigma_{2}$ are inner equivalent if and only if $U / K_{1}$ and $U / K_{2}$ are isomorphic as $U$-spaces.

There are two standard ways to classify involutions of $\mathfrak{u}$ : one is by means of Satake diagrams, the other by means of Vogan diagrams. In the following, we will recall the main ingredients of these classifications. Both classifications depend explicitly on the choice of a fixed Cartan subalgebra $\mathfrak{t} \subseteq \mathfrak{u}$ together with a positive root system $\Delta^{+} \subseteq \mathfrak{h}^{*}$, where $\mathfrak{h}=\mathfrak{t}^{\mathbb{C}} \subseteq \mathfrak{g}$. We write $W$ for the Weyl group and $I=\left\{\alpha_{1}, \ldots, \alpha_{l}\right\} \subseteq$ $\Delta^{+}$for the set of simple positive roots. When considering the associated Dynkin diagrams, we explicitly take $I$ as the set of vertices. Moreover, we fix as well Chevalley generators $\left\{e_{r}, f_{r}, h_{r} \mid r \in I\right\}$ for $\mathfrak{g}$ such that, under the $*$-structure on $\mathfrak{g}$ defining $\mathfrak{u}$, one has $e_{r}^{*}=f_{r}$ and $h_{r}^{*}=h_{r}$. In this case we say that the generators are compatible with the compact form $\mathfrak{u}$ of $\mathfrak{g}$, or that they are $*$-compatible. Note that with such a choice of generators the Chevalley automorphism

$$
\omega\left(h_{r}\right)=-h_{r}, \quad \omega\left(e_{r}\right)=-f_{r}, \quad \omega\left(f_{r}\right)=-e_{r} .
$$

is a $*$-automorphism of $\mathfrak{g}$.

\footnotetext{
${ }^{1} \mathrm{By}$ involution we will always mean a proper involution, i.e., not the identity.
} 
Denote by $T \subset U$ the maximal torus defined by $\mathfrak{t}$. For $\tau$ an automorphism of the Dynkin diagram, we denote as well by $\tau$ the corresponding *-preserving automorphism of $\mathfrak{g}$ determined by

$$
h_{r} \mapsto h_{\tau(r)}, \quad e_{r} \mapsto e_{\tau(r)}, \quad f_{r} \mapsto f_{\tau(r)} .
$$

For $X \subseteq I$, let $W_{X}$ be the subgroup of $W$ generated by the simple reflections $s_{r}$ corresponding to the roots $\alpha_{r}, r \in X$. We also view the $s_{r}$ as automorphisms of $\mathfrak{h}$ by duality, and we denote the canonical lift of the longest element $w_{X}$ to the normalizer $N_{U}(T)$ by $m_{X}$, so

$$
m_{X}=m_{r_{1}} \ldots m_{r_{k}}, \quad m_{r}=\exp \left(e_{r}\right) \exp \left(-f_{r}\right) \exp \left(e_{r}\right)
$$

if $w_{X}=s_{r_{1}} \ldots s_{r_{k}}$, as we have $s_{r}=\operatorname{Ad}\left(m_{r}\right)$.

Definition 2.4. We say that the involution $\theta$ is in Satake form (with respect to the chosen Chevalley presentation) if there exist an involutive automorphism $\tau$ of the Dynkin diagram, a globally $\tau$-invariant subset $X \subseteq I$ and unimodular numbers $\left(z_{r}\right)_{r \in I}$ such that

$$
\theta\left(h_{r}\right)=-\left(\operatorname{Ad} m_{X}\right)\left(h_{\tau(r)}\right), \quad \theta\left(e_{r}\right)=-\bar{z}_{\tau(r)}\left(\operatorname{Ad} m_{X}\right)\left(f_{\tau(r)}\right), \quad \theta\left(f_{r}\right)=-z_{\tau(r)}\left(\operatorname{Ad} m_{X}\right)\left(e_{\tau(r)}\right),
$$

where we assume that the following conditions are satisfied:

$$
\begin{aligned}
& \text { the action of } \tau \text { on } X \text { coincides with the action of }-w_{X} ; \\
& \qquad z_{r}=1 \text { for } r \in X .
\end{aligned}
$$

In other words, we can write

$$
\theta=(\operatorname{Ad} z) \circ\left(\operatorname{Ad} m_{X}\right) \circ \tau \circ \omega
$$

where $z \in T$ denotes any element such that $z\left(\alpha_{r}\right)=z_{r}$. If one defines a map $\theta$ by the formula (2.1), $\theta^{2}=\mathrm{id}$ is equivalent to

$$
z_{r} \bar{z}_{\tau(r)}=(-1)^{2\left(\alpha_{r}, \rho_{X}^{\vee}\right)}
$$

where $\rho_{X}^{\vee}$ is half the sum of the positive coroots of the root system generated by $X$, see [Kol14, Section 2.4]. In particular, we must have

$$
\left(\alpha_{r}, \rho_{X}^{\vee}\right) \in \mathbb{Z} \text { if } \tau(r)=r .
$$

Definition 2.5 ([Kol14, Definition 2.3]). A pair $(X, \tau)$, consisting of an involutive automorphism $\tau$ of the Dynkin diagram and a $\tau$-invariant subset $X \subsetneq I$ satisfying conditions $(2.2)$ and $(2.5)$ is called admissible $2^{2}$

From the above discussion, an involution in Satake form gives an admissible pair. Conversely, starting with an admissible pair we can choose *-compatible Chevalley generators $\left(e_{r}, f_{r}, h_{r}\right)_{r \in I}$ and numbers $z_{r} \in \mathbb{T}$ satisfying conditions 2.3) and (2.4), and define an involution in Satake form by 2.1). How exactly we choose $z_{r}$ is not important, since the involutions corresponding to different choices are conjugate by inner automorphisms defined by elements of $T$, or equivalently, we can obtain Satake forms of the same involution with different scalars $z_{r}$ by rescaling the generators $e_{r}$ and $f_{r}$. In particular, in the choice of $z_{r}$, besides satisfying (2.3) and (2.4), we can always set $z_{r}=1$ for all $r$ fixed by $\tau$. For every admissible pair $(X, \tau)$ we make such a choice and denote the corresponding element $z$ by $s(X, \tau)$ and the involution $(\operatorname{Ad} s(X, \tau)) \circ\left(\operatorname{Ad} m_{X}\right) \circ \tau \circ \omega$ by $\theta(X, \tau)$.

Note that an involution in Satake form preserves in particular the Cartan subalgebra $\mathfrak{h}$. Let $\Theta: \mathfrak{h}^{*} \rightarrow \mathfrak{h}^{*}$ define the involutive transformation dual to $\theta_{\mid \mathfrak{h}}$. The Satake diagram of $\theta$ is obtained from the Dynkin diagram as follows:

- the vertices corresponding to the simple roots in $X$ are painted black, and

- two distinct simple roots $\alpha_{r}$ and $\alpha_{s}$ in $I \backslash X$ such that $\Theta\left(\alpha_{r}\right)+\alpha_{s} \in \mathbb{Z} X$ are joined by an arrow.

Repeatedly using $\beta-s_{r} \beta=\left(\alpha_{r}^{\vee}, \beta\right) \alpha_{r}$, we obtain $\beta-w_{X}(\beta) \in \mathbb{Z} X$ for any $\beta$ in the root lattice $Q$. Combining this for $\beta=\Theta\left(\alpha_{r}\right)$ with the identity $-\left(w_{X} \circ \Theta\right)\left(\alpha_{r}\right)=\alpha_{\tau(r)}$, we see that the vertices $r$ and $s$ are joined by an arrow if and only if $r=\tau(s)$. Therefore the admissible pairs and the Satake diagrams contain literally the same information. Hence we will use these terminologies interchangeably.

Modulo the explicit statement about *-compatibility, the following result is the standard classification of symmetric pairs in terms of Satake diagrams as formulated, e.g., in [Kol14, Theorem 2.7]. For the benefit of the reader, we provide some further information on its proof in Appendix A.

\footnotetext{
${ }^{2}$ In Kol14 condition 2.5) is only stated for $i \in I \backslash X$, but for $i \in X$ one automatically has $\left(\rho_{X}^{\vee}, \alpha_{r}\right)=1$. We also exclude the case $X=I$ as it corresponds to the identity automorphism.
} 
Theorem 2.6. Let $\mathfrak{u}$ be a compact semisimple Lie algebra with fixed $*$-compatible Chevalley generators in $\mathfrak{g}=\mathfrak{u}^{\mathbb{C}}$. If $\sigma$ is an involution of $\mathfrak{u}$, there exists a Satake diagram $(X, \tau)$ such that $\sigma$ is inner equivalent with $\theta(X, \tau)$ for some, and hence any, choice of $s(X, \tau)$. Furthermore, up to an automorphism of the Dynkin diagram, $(X, \tau)$ depends only on the equivalence class of $\sigma$.

Next let us review Vogan diagrams.

Definition 2.7. We say that an involutive automorphism $\nu$ of $\mathfrak{u}$ is in Vogan form (with respect to a given set of Chevalley generators) if there exists an involutive automorphism $\mu$ of the Dynkin diagram such that

$$
\nu\left(h_{r}\right)=h_{\mu(r)}, \quad \nu\left(e_{r}\right)=\epsilon_{r} e_{\mu(r)}, \quad \nu\left(f_{r}\right)=\epsilon_{r} f_{\mu(r)}
$$

for some $\epsilon_{r}= \pm 1$ with $\epsilon_{r}=1$ if $\mu(r) \neq r$. We denote by $Y$ the set of simple roots fixed by $\mu$ such that $\epsilon_{r}=-1$.

A pair $(Y, \mu)$ as above, consisting of an involutive automorphism $\mu$ of the Dynkin diagram and a set $Y \subseteq I$ pointwise fixed by $\mu$, can be encoded as a Vogan diagram by making the vertices in $Y$ black and joining vertices $r, s$ by an arrow if $r=\mu(s)$, see Kna02, Section VI.8]. Therefore, we will refer to $(Y, \mu)$ itself as a Vogan diagram. We let $\nu(Y, \mu)$ be the involution associated to the Vogan diagram $(Y, \mu)$ (and a fixed set of Chevalley generators). We exclude the case where $\mu=$ id and $Y=\emptyset$, which corresponds to the identity map.

Again, the proof of the following theorem is provided in Appendix $\mathrm{A}$.

Theorem 2.8. Let $\mathfrak{u}$ be a compact semisimple Lie algebra with fixed $*$-compatible Chevalley generators in $\mathfrak{g}=\mathfrak{u}^{\mathbb{C}}$. If $\sigma$ is an involution of $\mathfrak{u}$, there exists a Vogan diagram $(Y, \mu)$ such that $\sigma$ is inner equivalent with $\nu(Y, \mu)$.

Remark 2.9. As opposed to Satake diagrams, the equivalence class of an involution can be described by non-isomorphic Vogan diagrams. We say that $(Y, \mu)$ is standard, and then that the corresponding involution $\nu=\nu(Y, \mu)$ is in standard Vogan form, if the following conditions are satisfied:

- every connected component of the Dynkin diagram contains at most one element of $Y$,

- if $r \in Y$ and $\mu=$ id on the connected component containing $r$, then $\left(\varpi_{r}-\varpi_{s}, \varpi_{s}\right) \leq 0$ for all $s$,

where $\varpi_{r}$ denote the fundamental weights. It can be shown that every involution $\nu$ of $u$ is inner equivalent with $\nu(Y, \mu)$ for a standard Vogan diagram $(Y, \nu)$. For this one starts with a Vogan form of $\nu$ and then makes a new, more careful choice of simple roots, see Kna02, Section VI.10]. Standard Vogan diagrams are much closer to being uniquely (up to conjugacy) associated to the conjugacy classes of involutions. There is, however, still some ambiguity, corresponding to the obvious isomorphisms $\mathfrak{s o}(p, q) \cong \mathfrak{s o}(q, p)$ and $\mathfrak{s p}(p, q) \cong \mathfrak{s p}(q, p)$ of real simple Lie algebras.

\section{Three Constructions of RibBon braided module $\mathrm{C}^{*}$-CATEGories From Symmetric Pairs}

Throughout this section $\mathfrak{u}$ will be a fixed compact semisimple Lie algebra with complexification $\mathfrak{g}=\mathfrak{u}^{\mathbb{C}}$ and a fixed set of Chevalley generators $\left\{e_{r}, f_{r}, h_{r} \mid r \in I\right\}$ compatible with $\mathfrak{u}$. We let $\mathfrak{h} \subseteq \mathfrak{g}$ be the associated Cartan subalgebra with root and weight lattices $Q$ and $P$, respectively, with positive part $Q^{+}$and $P^{+}$. We write the simple positive roots as $\alpha_{r}$, and the associated fundamental weights in $P^{+}$ as $\varpi_{r}$. We fix also a non-degenerate $\mathfrak{g}$-invariant form $(-,-)_{\mathfrak{g}}$ on $\mathfrak{g}$ for which the induced form $(-,-)$ on $\mathfrak{h}^{*}$ makes the short roots of each simple summand have square length 2 . We let $A=\left(a_{r s}\right)_{r s}$ be the associated Cartan matrix of $\mathfrak{g}$, with entries $a_{r s}=\left(\alpha_{r}^{\vee}, \alpha_{s}\right)=2\left(\alpha_{r}, \alpha_{s}\right) /\left(\alpha_{r}, \alpha_{r}\right)$, and write $d_{A}$ for the determinant of $A$ (for our purposes we may as well take the least common multiple of the determinants of Cartan matrices of irreducible components), which is also equal to the index of $Q$ in $P$. By our normalization, $(-,-)$ takes integral values on $Q \times Q$, and on $P \times P$ its values belong to $d_{A}^{-1} \mathbb{Z}$.

3.1. $q$-deformed tensor $\mathbf{C}^{*}$-categories. Let $\mathbf{q}$ be an indeterminate variable. We form the Hopf algebra $U_{\mathbf{q}}(\mathfrak{g})$ over the field $\mathbb{C}\left(\mathbf{q}^{1 / d_{A}}\right)$ of rational functions in $\mathbf{q}^{1 / d_{A}}$ using the same conventions as in Kol14, but taking the Cartan part to be labeled by elements of $P$, as follows. Let $U_{\mathbf{q}}\left(\mathfrak{n}^{+}\right)$be the algebra over $\mathbb{C}\left(\mathbf{q}^{1 / d_{A}}\right)$ generated by the elements $\left(E_{r}\right)_{r \in I}$ subject to the relations

$$
\sum_{n=0}^{1-a_{r s}}(-1)^{n}\left[\begin{array}{c}
1-a_{r s} \\
n
\end{array}\right]_{\mathbf{q}_{r}} E_{9}^{1-a_{r s}-n} E_{s} E_{r}^{n}=0 \quad(r \neq s),
$$


with $\mathbf{q}_{r}=\mathbf{q}^{\frac{1}{2}\left(\alpha_{r}, \alpha_{r}\right)}$. Let $U_{\mathbf{q}}\left(\mathfrak{n}^{-}\right)$be an isomorphic copy of $U_{\mathbf{q}}\left(\mathfrak{n}^{+}\right)$with generators $F_{r}$, and let $U_{\mathbf{q}}(\mathfrak{h})$ be the group algebra of $P$, spanned by the elements $\left(K_{\omega}\right)_{\omega \in P}$. Then $U_{\mathbf{q}}(\mathfrak{g})$ is defined as the algebra generated by $U_{\mathbf{q}}\left(\mathfrak{n}^{+}\right), U_{\mathbf{q}}\left(\mathfrak{n}^{-}\right)$and $U_{\mathbf{q}}(\mathfrak{h})$ with the following interchange relations:

$$
K_{\omega} E_{r}=\mathbf{q}^{\left(\omega, \alpha_{r}\right)} E_{r} K_{\omega}, \quad K_{\omega} F_{r}=\mathbf{q}^{-\left(\omega, \alpha_{r}\right)} F_{r} K_{\omega}, \quad\left[E_{r}, F_{s}\right]=\delta_{r s} \frac{K_{r}-K_{r}^{-1}}{\mathbf{q}_{r}-\mathbf{q}_{r}^{-1}},
$$

where $K_{r}=K_{\alpha_{r}}$. Multiplication gives a vector space isomorphism

$$
U_{\mathbf{q}}\left(\mathfrak{n}^{-}\right) \otimes U_{\mathbf{q}}(\mathfrak{h}) \otimes U_{\mathbf{q}}\left(\mathfrak{n}^{+}\right) \cong U_{\mathbf{q}}(\mathfrak{g})
$$

called the triangular decomposition, and this still holds with any of the factors on the left interchanged. One can endow $U_{\mathbf{q}}(\mathfrak{g})$ with a unique Hopf algebra structure such that

$$
\Delta\left(K_{\omega}\right)=K_{\omega} \otimes K_{\omega}, \quad \Delta\left(E_{r}\right)=E_{r} \otimes 1+K_{r} \otimes E_{r}, \quad \Delta\left(F_{r}\right)=F_{r} \otimes K_{r}^{-1}+1 \otimes F_{r} .
$$

We denote by $U_{\mathbf{q}}\left(\mathfrak{b}^{ \pm}\right)$the Hopf subalgebras generated by $U_{\mathbf{q}}\left(\mathfrak{n}^{ \pm}\right)$and $U_{\mathbf{q}}(\mathfrak{h})$.

By specializing $\mathbf{q}$ at $q \in \mathbb{C}^{\times}$not a root of unity, we obtain the Hopf algebra $U_{q}(\mathfrak{g})$ over $\mathbb{C}$. In case $0<q<1$, we can moreover endow $U_{q}(\mathfrak{g})$ with a good Hopf $*$-algebra structure

$$
E_{r}^{*}=F_{r} K_{r}, \quad F_{r}^{*}=K_{r}^{-1} E_{r}, \quad K_{\omega}^{*}=K_{\omega} .
$$

The resulting Hopf $*$-algebra will be denoted $U_{q}(\mathfrak{u}){ }^{3}$

We call a $U_{\mathbf{q}}(\mathfrak{g})$-module admissible if its restriction to $U_{\mathbf{q}}(\mathfrak{h})$ is a direct sum of one-dimensional modules of the form

$$
K_{\omega} \mapsto \mathbf{q}^{(\omega, \chi)}, \quad \chi \in P .
$$

It is called finite if it is finite-dimensional.

A similar definition can be made for $U_{q}(\mathfrak{g})$. For $U_{q}(\mathfrak{u})$ we moreover assume that the module is equipped with a pre-Hilbert space structure compatible with the $*$-structure, in which case we call it a *-representation. Finite admissible *-representations are then in one-to-one correspondence with finite *-representations under which each $K_{\omega}$ becomes a positive operator.

Definition 3.1. For $0<q<1$, we denote by $\operatorname{Rep}_{q}(\mathfrak{u})$ the tensor $\mathrm{C}^{*}$-category of finite admissible *-representations of the Hopf $*$-algebra $U_{q}(\mathfrak{u})$.

The equivalence classes of irreducible objects in $\operatorname{Rep}_{q}(\mathfrak{u})$ can be labeled by $\varpi \in P^{+}$, the set of dominant integral weights, and for each $\varpi \in P^{+}$one can canonically construct an irreducible $*$-representation $V_{\varpi}^{q}$ generated by a highest weight vector $\xi_{\varpi}$ vanishing under the $E_{r}$ and with

$$
K_{\chi} \xi_{\varpi}=q^{(\chi, \varpi)} \xi_{\varpi}
$$

We can then make the (non-unital) direct sum $*$-algebra

$$
\mathscr{U}_{q}(\mathfrak{u})=\bigoplus_{\varpi \in P^{+}} B\left(V_{\varpi}^{q}\right)
$$

into a discrete multiplier $*$-bialgebra $\left(\mathscr{U}_{q}(\mathfrak{u}), \Delta_{q}\right)$ with dense, coproduct-compatible embedding

$$
U_{q}(\mathfrak{u}) \subseteq M\left(\mathscr{U}_{q}(\mathfrak{u})\right)
$$

There is a unique quasi-triangular structure $\mathscr{R}_{q} \in U_{q}(\mathfrak{u}) \hat{\otimes} U_{q}(\mathfrak{u})$ such that ${ }^{4}$ for all $\varpi, \chi \in P^{+}$

$$
\mathscr{R}_{q}\left(\xi_{\varpi} \otimes \eta_{w_{0} \chi}\right)=q^{-\left(\varpi, w_{0} \chi\right)} \xi_{\varpi} \otimes \eta_{w_{0} \chi},
$$

where $\eta_{w_{0} \chi}$ is the lowest weight vector in $V_{\chi}^{q}$ of weight $w_{0} \chi$ and $w_{0}$ is the longest element in the Weyl group of $\mathfrak{g}$. Correspondingly, $\operatorname{Rep}_{q}(\mathfrak{u})$ becomes a braided tensor $\mathrm{C}^{*}$-category.

An equivalent braided tensor $\mathrm{C}^{*}$-category can be obtained from monodromy of Knizhnik-Zamolodchikov equations. Specifically, let $\left\{X_{i}\right\}_{i \in \mathcal{I}}$ be an orthonormal basis of $\mathfrak{g}$ with respect to the Hermitian inner product $\langle X, Y\rangle_{\mathfrak{g}}=\left(X, Y^{*}\right)_{\mathfrak{g}}$, and put

$$
t=\sum_{i \in \mathcal{I}} X_{i}^{*} \otimes X_{i} \in \operatorname{Sym}^{2}(\mathfrak{g})^{\mathfrak{g}} .
$$

\footnotetext{
${ }^{3}$ The theory can also be developed for $q>1$, but this case would need a slight modification in later sections, so we restrict to $0<q<1$ from the outset. Note that the $*$-structure is also well-defined for $q<0$, but the resulting $*$-algebra does not have a good $*$-representation theory.

${ }^{4}$ Our $\mathscr{R}_{q}$ corresponds to $\mathscr{R}_{21}^{-1}$ in NT11a as to have the same conventions for the $R$-matrix as in Kol14.
} 
Let $V_{1}, \ldots, V_{n}$ be finite-dimensional $\mathfrak{u}$-representations, and $\hbar \in i \mathbb{R}$. On the configuration space of $n$ distinct complex numbers

$$
\Omega_{n}=\left\{z \in \mathbb{C}^{n} \mid \prod_{i \neq j}\left(z_{i}-z_{j}\right) \neq 0\right\}
$$

we consider the following system of differential equations on $V_{1} \otimes \cdots \otimes V_{n}$-valued functions, called $K Z_{n}$ equations (of type A),

$$
\frac{\partial v}{\partial z_{i}}=\hbar \sum_{j \neq i} \frac{t_{i j}}{z_{i}-z_{j}} v \quad(i=1, \ldots, n),
$$

where $t_{i j}$ means $t$ acting on the $i$-th and $j$-th tensors. Then the appropriately normalized monodromy of $\mathrm{KZ}_{3}$ from the region $\left|z_{2}-z_{1}\right| \ll\left|z_{3}-z_{1}\right|$ to the region $\left|z_{3}-z_{2}\right| \ll\left|z_{3}-z_{1}\right|$ then gives a family of unitary operators

$$
\Phi_{\hbar}:(V \otimes W) \otimes Z \rightarrow V \otimes(W \otimes Z),
$$

providing a non-trivial unitary associator on the tensor $\mathrm{C}^{*}$-category $\operatorname{Rep}(\mathfrak{u})$ of finite-dimensional unitary representations of $\mathfrak{u}$, see NT11a. This becomes a braided tensor $\mathrm{C}^{*}$-category with respect to the braiding $^{5}$ defined by $\Sigma e^{-\pi i \hbar t}$, where $\Sigma$ is the flip map. Correspondingly, if we write $U(\mathfrak{u})$ for the enveloping Hopf algebra of $\mathfrak{g}$ with the $*$-structure induced by $\mathfrak{u}$, together with its dense embedding

$$
U(\mathfrak{u}) \subseteq M(\mathscr{U}(\mathfrak{u})), \quad \mathscr{U}(\mathfrak{u})=\bigoplus_{\varpi \in P^{+}} B\left(V_{\varpi}\right),
$$

where again $V_{\varpi}$ is constructed with respect to the fixed Chevalley generators, then $U(\mathfrak{u})$ becomes a quasitriangular quasi-Hopf algebra with respect to $\phi_{\hbar} \in U(\mathfrak{u})^{\hat{\otimes} 3}$ defined by $\Phi_{\hbar}$ and $\mathscr{R}_{\hbar}=e^{-\pi i \hbar t} \in U(\mathfrak{u})^{\hat{\otimes} 2}$. We denote by $\operatorname{Rep}_{\hbar}(\mathfrak{u})$ the corresponding braided tensor $\mathrm{C}^{*}$-category.

One has the following result.

Theorem 3.2 ( NT11a], cf. Dri89, KL94a, KL94b]). Let $0<q<1$, and let $\hbar \in i \mathbb{R}_{>0}$ be such that $q=e^{\pi i \hbar}$. Then there exists an equivalence of braided tensor $C^{*}$-categories

$$
F: \operatorname{Rep}_{\hbar}(\mathfrak{u}) \rightarrow \operatorname{Rep}_{q}(\mathfrak{u})
$$

such that $F\left(V_{\varpi}\right)=V_{\varpi}^{q}$.

The above equivalence is, moreover, unique up to a natural unitary monoidal isomorphism NT11b, NT12. In particular, the Tannaka reconstruction applied to the forgetful functor on $\operatorname{Rep}_{q}(\mathfrak{u})$ composed with any $F$ as above will produce a discrete multiplier Hopf $*$-algebra with a distinguished isomorphism to $\left(\mathscr{U}_{q}(\mathfrak{u}), \Delta_{q}\right)$.

3.2. Ribbon braided module $\mathbf{C}^{*}$-categories from $\mathbf{K Z}$ equations of type $\mathbf{B}$. Let $\sigma$ be an involution on $\mathfrak{u}$ (recall that we assume involutions to be nontrivial, so $\sigma \neq \mathrm{id}$ ). In this subsection, we will construct from $\sigma$ a ribbon $\sigma$-braided module $\mathrm{C}^{*}$-category over $\operatorname{Rep}_{\hbar}(\mathfrak{u})$.

Let $\mathfrak{g}_{ \pm}$be the \pm 1 -eigenspaces in $\mathfrak{g}$ for $\sigma$, and write $\mathfrak{u}_{ \pm}=\mathfrak{g}_{ \pm} \cap \mathfrak{u}$. We will also write

$$
\mathfrak{u}_{+}=\mathfrak{k}, \quad \mathfrak{u}_{-}=\mathfrak{m}, \quad \text { so } \quad \mathfrak{g}_{+}=\mathfrak{k}^{\mathbb{C}}, \quad \mathfrak{g}_{-}=\mathfrak{m}^{\mathbb{C}} .
$$

Let $\left\{X_{i}\right\}_{i \in \mathcal{I}_{ \pm}}$be an orthonormal basis of $\mathfrak{g}_{ \pm}$for the restriction of Hermitian inner product on $\mathfrak{g}$ as in the previous section, and put

$$
t^{+}=t^{\mathfrak{k}}=\sum_{i \in \mathcal{I}_{+}} X_{i}^{*} \otimes X_{i} \in \mathfrak{k}^{\mathbb{C}} \otimes \mathfrak{k}^{\mathbb{C}}, \quad t^{-}=t^{\mathfrak{m}}=\sum_{i \in \mathcal{I}_{-}} X_{i}^{*} \otimes X_{i} \in \mathfrak{m}^{\mathbb{C}} \otimes \mathfrak{m}^{\mathbb{C}}
$$

so that $t=t^{+}+t^{-}$. Finally, let us denote the Casimir element of $\mathfrak{k}$ as

$$
C^{+}=C^{\mathfrak{k}}=\sum_{i \in \mathcal{I}_{+}} X_{i}^{*} X_{i} \in U\left(\mathfrak{k}^{\mathbb{C}}\right) .
$$

The following definition introduces the Knizhnik-Zamolodchikov equations associated to the hyperplane arrangement of the Coxeter group of type B, as constructed from $\sigma$ in Bro12, Section 1.3].

\footnotetext{
${ }^{5}$ Again, we choose the opposite braiding of NT11a.
} 
Definition 3.3 ( Che89, Lei94,GL00]). Let $\hbar \in \mathbb{C}$,

$$
\Omega_{n}^{\prime}=\left\{w \in \mathbb{C}^{n} \mid \prod_{ \pm, i \neq j} w_{i}\left(w_{i} \pm w_{j}\right) \neq 0\right\},
$$

$V_{0}$ be a finite-dimensional representation of $\mathfrak{k}$, and $V_{1}, \ldots, V_{n}$ finite-dimensional representations of $\mathfrak{u}$. The system of 2-cyclotomic $K Z_{n}$-equations is the following system of differential equations on $V_{0} \otimes \cdots \otimes V_{n^{-}}$ valued functions on $\Omega_{n}^{\prime}$ :

$$
\frac{\partial v}{\partial w_{i}}=\hbar\left(\frac{2 t_{0, i}^{\mathfrak{k}}+C_{i}^{\mathfrak{k}}}{w_{i}}+\sum_{ \pm, j \neq i} \frac{t_{i, j}^{\mathfrak{k}} \pm t_{i, j}^{\mathfrak{m}}}{w_{i} \mp w_{j}}\right) v, \quad(i=1, \ldots, n) .
$$

Note that the coefficients of the above system indeed lie in $U\left(\mathfrak{k}^{\mathbb{C}}\right) \otimes U(\mathfrak{g})^{\otimes n}$, so that the equations are meaningful. Let us check explicitly that this is a compatible system of equations.

Lemma 3.4. The 2-cyclotomic $K Z_{n}$-system (3.1) is flat for any $n \geq 2$.

Proof. We verify that the conditions 1-4) of Lei94, Proposition 1.2] are satisfied with

$$
\tau_{i j}=t_{i j}, \quad \quad \mu_{i j}=t_{i j}^{\mathfrak{k}}-t_{i j}^{\mathfrak{m}}=(\mathrm{id} \otimes \sigma)(t)_{i j}, \quad \nu_{i}=2 t_{0 i}^{\mathfrak{k}}+C_{i}^{\mathfrak{k}} .
$$

Equations 1) and 2.a) follow from $[t, \Delta(X)]=0$ for all $X \in \mathfrak{g}$. Equation 2.b) follows from

$$
\begin{aligned}
{\left[\mu_{i k}, \tau_{i j}+\mu_{j k}\right] } & =\left[(\operatorname{id} \otimes \sigma)(t)_{i k}, t_{j i}+(\mathrm{id} \otimes \sigma)(t)_{j k}\right] \\
& =\sigma_{k}\left(\left[t_{i k}, t_{j i}+t_{j k}\right]\right) \\
& =0
\end{aligned}
$$

where we used that $\sigma$ is a Lie algebra automorphism.

For 3.a), we use that $\Delta\left(C^{\mathfrak{k}}\right)=\left(C^{\mathfrak{k}} \otimes 1\right)+\left(1 \otimes C^{\mathfrak{k}}\right)+2 t^{\mathfrak{k}}$ to compute that

$$
\begin{aligned}
{\left[\tau_{i j}+\nu_{i}+\nu_{j}, \mu_{i j}\right] } & =\left[t_{i j}+C_{i}^{\mathfrak{k}}+C_{j}^{\mathfrak{k}}+2 t_{0, i}^{\mathfrak{k}}+2 t_{0, j}^{\mathfrak{k}}, t_{i j}^{\mathfrak{k}}-t_{i j}^{\mathfrak{m}}\right] \\
& =\left[-\left(t_{i j}^{\mathfrak{k}}-t_{i j}^{\mathfrak{m}}\right)+\Delta\left(C^{\mathfrak{k}}\right)_{i j}+2(\mathrm{id} \otimes \Delta)\left(t^{\mathfrak{k}}\right)_{0 i j}, t_{i j}^{\mathfrak{k}}-t_{i j}^{\mathfrak{m}}\right] \\
& =0,
\end{aligned}
$$

using also that $\left[t^{\mathfrak{k}}, \Delta(X)\right]=0$ and hence $\left[t^{\mathfrak{m}}, \Delta(X)\right]=0$ for all $X \in \mathfrak{k}^{\mathbb{C}}$. Then 3.b) follows from applying $\sigma$ to the $j$-th leg.

For 3.c), we compute

$$
\begin{aligned}
{\left[\tau_{i j}+\nu_{i}+\mu_{i j}, \nu_{j}\right] } & =\left[2 t_{i j}^{\mathfrak{k}}+2 t_{0 i}^{\mathfrak{k}}+C_{i}^{\mathfrak{k}}, 2 t_{0 j}^{\mathfrak{k}}+C_{j}^{\mathfrak{k}}\right] \\
& =\left[2(\Delta \otimes \mathrm{id})\left(t^{\mathfrak{k}}\right)_{0, j, i}+C_{i}^{\mathfrak{k}}, 2 t_{0 j}^{\mathfrak{k}}+C_{j}^{\mathfrak{k}}\right] \\
& =0 .
\end{aligned}
$$

Equations of 4) are obvious.

We will be particularly interested in the 2-cyclotomic $\mathrm{KZ}_{2}$-equation. Analogously to the $\mathrm{KZ}_{3}$-equation of type $\mathrm{A}$, we can construct a modified 2-cyclotomic $\mathrm{KZ}_{2}$-equation Enr07 on $\mathbb{C} \backslash\{0, \pm 1\}$, with values in $V_{0} \otimes V_{1} \otimes V_{2}$ :

$$
H^{\prime}(w)=\hbar\left(\frac{B_{-}}{w+1}+\frac{2 A}{w}+\frac{B_{+}}{w-1}\right) H(w),
$$

where

$$
A=t_{01}^{\mathfrak{k}}+\frac{1}{2} C_{1}^{\mathfrak{k}}, \quad B_{-}=t_{12}^{\mathfrak{k}}-t_{12}^{\mathfrak{m}}, \quad B_{+}=t_{12}^{\mathfrak{k}}+t_{12}^{\mathfrak{m}}=t_{12} .
$$

If $H$ is a solution of the modified 2-cyclotomic $\mathrm{KZ}_{2}$-equation, we see by using Lemma 3.7 below that, with

$$
d=\hbar\left(2 t_{01}^{\mathfrak{k}}+2 t_{02}^{\mathfrak{k}}+2 t_{12}^{\mathfrak{k}}+C_{1}^{\mathfrak{k}}+C_{2}^{\mathfrak{k}}\right)
$$

the function

$$
v\left(w_{1}, w_{2}\right)=w_{2}^{d} H\left(w_{1} / w_{2}\right)
$$

is a solution of the 2-cyclotomic $\mathrm{KZ}_{2}$-equation. 
Remark 3.5. The modified $\mathrm{KZ}_{2}$-equation can be written in a more concise form by considering rather

$$
G(z)=H(\sqrt{z})
$$

on $\mathbb{C} \backslash \mathbb{R}^{-}$, which satisfies the equation

$$
G^{\prime}(z)=\hbar\left(\frac{A}{z}+\frac{B(z)}{z-1}\right) G(z), \quad \text { with } B(z)=t_{12}^{\mathfrak{k}}+\frac{t_{12}^{\mathfrak{m}}}{\sqrt{z}} .
$$

The second term is precisely $X(z) / z(z-1)$ in the notation of EE05], but we have swapped the first and the last leg and started indexing at 0 as to have right module category actions later on. We also chose a different cut for the $\theta_{k}$ than the set $\mathbf{D}$ in EE05, as it allows for a more concise form of their term $X(z)$.

Following Enr07] we define $\Psi$ as a normalized monodromy of 3.2 from 0 to 1.6 Namely, consider general operators $a, b_{+}$and $b_{-}$on a finite dimensional vector space and the equation

$$
H^{\prime}(w)=\left(\frac{b_{-}}{w+1}+\frac{a}{w}+\frac{b_{+}}{w-1}\right) H(w) .
$$

Then from the theory of differential equations with singularities it is known (see, e.g., Was87, NT11a, Section 2]) that if the operators $a$ and $b_{+}$have no eigenvalues that differ by a nonzero integer, then there exist unique operator valued solutions $H_{0}$ and $H_{1}$ of this equation on $(0,1)$ such that

- $H_{0}(w) w^{-a}$ is analytic in a neighborhood of 0 and equal to id at 0 ,

- $H_{1}(w)(1-w)^{-b_{+}}$is analytic in a neighborhood of 1 and is equal to id at 1 .

We then put

$$
\Psi\left(a, b_{+}, b_{-}\right)=H_{1}(w)^{-1} H_{0}(w),
$$

which is independent of $w \in(0,1)$. Note that if $\hbar$ is purely imaginary and $a, b_{+}, b_{-}$are Hermitian, the associated monodromy $\Psi\left(a, b_{+}, b_{-}\right)$will be unitary.

Assume now that $\hbar \in i \mathbb{R}$. Let $V, W$ be finite dimensional unitary representations of $\mathfrak{u}$, and $X$ a finite dimensional unitary representation of $\mathfrak{k}$. Then the skew-adjoint operators $2 \hbar A$ and $\hbar B_{ \pm}$from (3.3), considered as operators on $X \otimes V \otimes W$, have no eigenvalues that differ by a nonzero integer, so we get a unitary

$$
\Psi_{X, V, W}=\Psi\left(\left.2 \hbar A\right|_{X \otimes V \otimes W},\left.\hbar B_{+}\right|_{X \otimes V \otimes W},\left.\hbar B_{-}\right|_{X \otimes V \otimes W}\right) \in B(X \otimes V \otimes W) .
$$

By slightly abusing notation, we can write the family $\left(\Psi_{X, V, W}\right)_{X, V, W}$ as

$$
\Psi=\Psi\left(\hbar\left(2 t_{01}^{\mathfrak{k}}+C_{1}^{\mathfrak{k}}\right), \hbar t_{12}, \hbar\left(t_{12}^{\mathfrak{k}}-t_{12}^{\mathfrak{m}}\right)\right) .
$$

Write $\operatorname{Rep}(\mathfrak{k})$ for the category of finite dimensional unitary representations of $\mathfrak{k}$. We have the obvious functor

$$
\odot: \operatorname{Rep}(\mathfrak{k}) \times \operatorname{Rep}(\mathfrak{u}) \rightarrow \operatorname{Rep}(\mathfrak{k})
$$

of tensor product of representations of $\mathfrak{k}$ with the restrictions of representations of $\mathfrak{u}$ to $\mathfrak{k}$. Then the morphisms $\Psi_{X, V, W}$ can be regarded as natural isomorphisms

$$
\Psi_{X, V, W}:(X \odot V) \odot W \rightarrow X \odot(V \otimes W),
$$

making $\operatorname{Rep}(\mathfrak{k})$ into a right $\operatorname{Rep}_{\hbar}(\mathfrak{u})$-module $C^{*}$-category which we will denote $\operatorname{Rep}{ }_{\hbar}(\mathfrak{k})$, see EE05, Theorem 4.6; Enr07, Proposition 2.1] 7

In Enr07, Section 4.5], it is essentially proved that $\operatorname{Rep}_{\hbar}(\mathfrak{k})$ is a braided module category for the braid given by the operator $e^{-\pi i \hbar\left(2 t_{01}^{\mathfrak{k}}+C_{1}^{\mathfrak{k}}\right)}$. Since Enriquez works with crossed products (see our Remark 1.13 ) and does not consider the ribbon braid relation, let us provide some details.

Proposition 3.6. For any operators $a, b_{+}, b_{-}$on a finite dimensional vector space we have the following identity, whenever the left hand side of it is well-defined:

$$
\Psi\left(a, b_{+}, b_{-}\right)^{-1} e^{\pi i b_{+}} \Psi\left(c, b_{+}, b_{-}\right) e^{\pi i c} \Psi\left(c, b_{-}, b_{+}\right)^{-1} e^{\pi i b_{-}} \Psi\left(a, b_{-}, b_{+}\right) e^{\pi i a}=1,
$$

where $c=-a-b_{+}-b_{-}$.

Proof. This is a particular case of Enr07, Proposition 2.1]. To be pedantic, Enriquez works with formal power series, but whenever all the terms in the above identity can be defined analytically, all his equalities can be specialized.

\footnotetext{
${ }^{6}$ It is also possible to define $\Psi$ as a normalized monodromy of 3.4 from 0 to 1 , but then we have to be careful with normalization, since the natural normalization, as in EE05., leads to an operator which differs from $\Psi$ by the factor $2^{\hbar t_{12}}$.

${ }^{7}$ Note once again that the normalizations of monodromy operators in these papers are different. We use the one of the latter.
} 
We will apply this proposition to

$$
a=2 \hbar A=\hbar\left(2 t_{01}^{\mathfrak{k}}+C_{1}^{\mathfrak{k}}\right), \quad b_{+}=\hbar B_{+}=\hbar t_{12}, \quad b_{-}=\hbar B_{-}=\hbar\left(t_{12}^{\mathfrak{k}}-t_{12}^{\mathfrak{m}}\right)
$$

acting on $X \otimes V \otimes W$.

Lemma 3.7. Define

$$
d=\hbar\left(2 t_{01}^{\mathfrak{k}}+2 t_{02}^{\mathfrak{k}}+2 t_{12}^{\mathfrak{k}}+C_{1}^{\mathfrak{k}}+C_{2}^{\mathfrak{k}}\right) .
$$

Then $d$ commutes with $a, b_{+}$and $b_{-}$. Furthermore, with $c=-a-b_{+}-b_{-}$, we have the following identities:

$$
d+c=a_{02}=\hbar\left(2 t_{02}^{\mathfrak{k}}+C_{2}^{\mathfrak{k}}\right), \quad d-a=(\Delta \otimes \mathrm{id})(a), \quad d=(\operatorname{id} \otimes \Delta)(a) .
$$

Proof. As $t_{01}^{\mathfrak{k}}+t_{02}^{\mathfrak{k}}=(\mathrm{id} \otimes \Delta)\left(t^{\mathfrak{k}}\right)$ and $t_{02}^{\mathfrak{k}}+t_{12}^{\mathfrak{k}}=(\Delta \otimes \mathrm{id})\left(t^{\mathfrak{k}}\right)$, and $t^{\mathfrak{k}}$ is $\mathfrak{k}^{\mathbb{C}}$-invariant, it follows that $d$ commutes with $t_{12}^{\mathfrak{k}}$ and $t_{01}^{\mathfrak{k}}$. Clearly $d$ also commutes with $C_{1}^{\mathfrak{k}}$. It remains to check that $d$ commutes with $t_{12}$. The summand $t_{01}^{\mathfrak{k}}+t_{02}^{\mathfrak{k}}$ commutes with $t_{12}$ as $t$ is $\mathfrak{g}$-invariant. For the same reason, $t_{12}$ commutes with $2 t_{12}^{\mathfrak{k}}+C_{1}^{\mathfrak{k}}+C_{2}^{\mathfrak{k}}=\Delta\left(C^{\mathfrak{k}}\right)$. Identities (3.7) are straightforward from the equalities we have indicated in this proof.

Theorem 3.8. For $\hbar \in i \mathbb{R}$, the triple $\operatorname{Rep}_{\hbar}(\mathfrak{k})=\left(\operatorname{Rep}\left(\mathfrak{k}^{\mathbb{C}}\right), \odot, \Psi, e^{-\pi i \hbar\left(2 t_{01}^{\mathfrak{k}}+C_{1}^{\mathfrak{k}}\right)}\right)$ is a ribbon $\sigma$-braided right module $C^{*}$-category over $\operatorname{Rep}_{\hbar}(\mathfrak{u})=\left(\operatorname{Rep}(\mathfrak{u}), \otimes, \Phi, \Sigma e^{-\pi i \hbar t}\right)$.

Proof. Let $H(w)$ be a solution of the equation (3.5). If $D$ is an operator commuting with all $a, b_{-}, b_{+}$, one easily sees that $H_{D}(w)=H(w) z^{D}$ is a solution of 3.5 with $a$ replaced by $a+D$. In particular, $\Psi\left(a, b_{+}, b_{-}\right)=\Psi\left(a+D, b_{+}, b_{-}\right)$. Hence, as $c=a_{02}-d$, with $d$ commuting with $c, b_{+}, b_{-}$, it follows that we can write $(3.6)$ as

$$
\Psi\left(a, b_{+}, b_{-}\right)^{-1} e^{\pi i b_{+}} \Psi\left(a_{02}, b_{+}, b_{-}\right) e^{\pi i a_{02}} \Psi\left(a_{02}, b_{-}, b_{+}\right)^{-1} e^{\pi i b_{-}} \Psi\left(a, b_{-}, b_{+}\right) e^{\pi i a}=e^{\pi i d} .
$$

We have $\Psi\left(a, b_{+}, b_{-}\right)=\Psi$ and $\Psi\left(a_{02}, b_{+}, b_{-}\right)=\Psi_{021}$. As (id $\left.\otimes \mathrm{id} \otimes \sigma\right)\left(b_{+}\right)=b_{-}$, we also have

$$
\Psi\left(a_{02}, b_{-}, b_{+}\right)=(\mathrm{id} \otimes \mathrm{id} \otimes \sigma)\left(\Psi_{021}\right) \quad \text { and } \Psi\left(a, b_{-}, b_{+}\right)=(\mathrm{id} \otimes \mathrm{id} \otimes \sigma)(\Psi) .
$$

Hence we get

$$
\Psi^{-1} e^{\pi i b_{+}} \Psi_{021} e^{\pi i a_{02}}(\mathrm{id} \otimes \mathrm{id} \otimes \sigma)\left(\Psi_{021}^{-1} e^{\pi i b_{+}} \Psi\right) e^{\pi i a}=e^{\pi i d} .
$$

Let us now verify that this entails that $\mathscr{E}=e^{-\pi i a}=e^{-\pi i \hbar\left(2 t_{01}^{\mathrm{k}}+C_{1}^{\mathrm{k}}\right)}$ is a ribbon $\sigma$-braid with respect to $\mathscr{R}=e^{-\pi i b_{+}}=e^{-\pi i \hbar t}$. Clearly $\mathscr{E}$ commutes with $\Delta(X)=($ id $\otimes \sigma) \Delta(X)$ for all $X \in U(\mathfrak{k})$. If in (3.8) we move $e^{\pi i a}$ to the right, take inverses and apply $\sigma$ to the last leg, we obtain (using the identity $d-a=(\Delta \otimes \mathrm{id})(a))$ the $\sigma$-octagon relation

$$
\Psi^{-1} \mathscr{R}_{21} \Psi_{021} \mathscr{E}_{02}(\mathrm{id} \otimes \mathrm{id} \otimes \sigma)\left(\Psi_{021}^{-1} \mathscr{R}_{12} \Psi\right)=(\Delta \otimes \mathrm{id})(\mathscr{E}) .
$$

Using the relations of Lemma 3.7 and that $d$ commutes with $a$, we then obtain

$$
\Psi^{-1} \mathscr{R}_{21} \Psi_{021} \mathscr{E}_{02}(\mathrm{id} \otimes \mathrm{id} \otimes \sigma)\left(\Psi_{021}^{-1} \mathscr{R}_{12} \Psi\right) \mathscr{E}_{01}=(\mathrm{id} \otimes \Delta)(\mathscr{E}),
$$

which is equivalent to the ribbon $\sigma$-braid equation as $\Psi=($ id $\otimes \sigma \otimes \sigma)(\Psi)$ commutes with (id $\otimes \Delta)(\mathscr{E})$.

Up till now, the choice of a fixed set of Chevalley generators has not played any rôle. However, recall that this choice determined the particular form of the braided tensor equivalence

$$
\operatorname{Rep}_{\hbar}(\mathfrak{u}) \rightarrow \operatorname{Rep}_{q}(\mathfrak{u}), \quad q=e^{\pi i \hbar} .
$$

By means of this equivalence, any braided autoequivalence of $\operatorname{Rep}_{\hbar}(\mathfrak{u})$ can be transported to a braided autoequivalence of $\operatorname{Rep}_{q}(\mathfrak{u})$, and any ribbon twist-braided module $\mathrm{C}^{*}$-category for $\operatorname{Rep}_{\hbar}(\mathfrak{u})$ correspondingly defines one for $\operatorname{Rep}_{q}(\mathfrak{u})$. In particular, we can interpret in this way $\operatorname{Rep}_{\hbar}(\mathfrak{k})$ as a ribbon $\sigma$-braided module $\mathrm{C}^{*}$-category for $\operatorname{Rep}_{q}(\mathfrak{u})$.

Remark 3.9. Note that if $\sigma$ and $\sigma^{\prime}$ are inner equivalent, there is an obvious isomorphism between the associated twist-braided module $\mathrm{C}^{*}$-categories. 
3.3. Ribbon braided module $\mathbf{C}^{*}$-categories from coideal quantum symmetric pairs. Fix again a compact semisimple Lie algebra $\mathfrak{u}$ together with compatible Chevalley generators $\left\{e_{r}, f_{r}, h_{r} \mid r \in I\right\}$ of $\mathfrak{g}=\mathfrak{u}^{\mathbb{C}}$. Fix also an admissible pair $(X, \tau)$ with associated involution $\theta=\theta(X, \tau)$ and the fixed point Lie algebra $\mathfrak{k}=\mathfrak{u}^{\theta}$. Then $\theta$ has a quantum analogue [Kol14, Definition 4.3]

$$
\theta_{\mathbf{q}}=\theta_{\mathbf{q}}(X, \tau)=(\operatorname{Ad} s(X, \tau)) \circ T_{w_{X}} \circ \psi \circ \tau \circ \omega: U_{\mathbf{q}}(\mathfrak{g}) \rightarrow U_{\mathbf{q}}(\mathfrak{g}),
$$

where the different maps in the composition are all $\mathbb{C}\left(\mathbf{q}^{1 / d}\right)$-algebra automorphisms given by the following formulas:

- $\omega$ is the quantum Chevalley automorphism

$$
\omega\left(E_{r}\right)=-F_{r}, \quad \omega\left(F_{r}\right)=-E_{r}, \quad \omega\left(K_{\chi}\right)=K_{-\chi},
$$

- $\tau$ is the automorphism induced by the Dynkin diagram automorphism,

$$
\tau\left(E_{r}\right)=E_{\tau(r)}, \quad \tau\left(F_{r}\right)=F_{\tau(r)}, \quad \tau\left(K_{\varpi_{r}}\right)=K_{\varpi_{\tau(r)}},
$$

- $\psi$ is the automorphism

$$
\psi\left(E_{r}\right)=E_{r} K_{r}, \quad \psi\left(F_{r}\right)=K_{r}^{-1} F_{r}, \quad \psi\left(K_{\chi}\right)=K_{\chi},
$$

- $T_{w_{X}}$ is the Lusztig braid operator (following the conventions of [Jan96, Section 8], see also Remark in loc. cit. Section 8.6) corresponding to the longest element in the Weyl group associated to $X$ : with a reduced expression

$$
w_{X}=s_{r_{1}} \ldots s_{r_{M}}
$$

we have

$$
T_{w_{X}}=T_{r_{1}} \ldots T_{r_{M}}
$$

where each $T_{r}$ is the algebra automorphisms determined by

$$
T_{r}\left(E_{r}\right)=-F_{r} K_{r}, \quad T_{r}\left(F_{r}\right)=-K_{r}^{-1} E_{r}, \quad T_{r}\left(K_{\chi}\right)=K_{s_{r}(\chi)}
$$

and for $r \neq s$

$$
T_{r}\left(E_{s}\right)=\sum_{m+n=-a_{r s}} \frac{\left(-\mathbf{q}_{r}\right)^{-m}}{[m]_{\mathbf{q}_{r}} ![n]_{\mathbf{q}_{r}} !} E_{r}^{n} E_{s} E_{r}^{m}, \quad T_{r}\left(F_{s}\right)=\sum_{m+n=-a_{r s}} \frac{\left(-\mathbf{q}_{r}\right)^{m}}{[m]_{\mathbf{q}_{r}} ![n]_{\mathbf{q}_{r}} !} F_{r}^{m} F_{s} F_{r}^{n},
$$

- $\operatorname{Ad}(s(X, \tau))$ is given by

$$
E_{r} \mapsto z_{r} E_{r}, \quad F_{r} \mapsto z_{r}^{-1} F_{r}, \quad K_{\chi} \mapsto K_{\chi}
$$

where $z_{r}=s(X, \tau)_{r}$.

The same construction applies verbatim to construct an automorphism $\theta_{q}$ of $U_{q}(\mathfrak{g})$ with $q \in \mathbb{C} \backslash\{0\}$ not a root of unity. Note however that $\theta_{\mathbf{q}}$ is not involutive and does not preserve the comultiplication. Furthermore, $\theta_{q}$ does not preserve the $*$-structure when $0<q<1$.

Associated to $(X, \tau)$ one has a (family of) right coideal subalgebras

$$
B \subseteq U_{\mathbf{q}}(\mathfrak{g}), \quad \Delta(B) \subseteq B \otimes U_{\mathbf{q}}(\mathfrak{g}) .
$$

To introduce them, we further borrow notation from Kol14]. Let us first recall the parameter sets $\mathcal{C}_{\mathbf{q}}$ and $\mathcal{S}_{\mathbf{q}}$. Writing

$$
I_{\mathcal{C}}=\left\{r \in I \backslash X \mid \tau(r) \neq r \text { and }\left(\alpha_{r}, \Theta\left(\alpha_{r}\right)\right)=0\right\}
$$

we put

$$
\mathcal{C}_{\mathbf{q}}=\left\{\left(c_{r}(\mathbf{q})\right)_{r \in I \backslash X} \mid c_{r}(\mathbf{q}) \in \mathbb{C}\left(\mathbf{q}^{1 / d}\right)^{\times} \text {and } c_{r}(\mathbf{q})=c_{\tau(r)}(\mathbf{q}) \text { for } r \in I_{\mathcal{C}}\right\} .
$$

(Recall that $\Theta$ denotes the involutive transformation dual to $\theta_{\mid \mathfrak{h}}$.) Note that by Hel01, Chapter X, Exercises and further results, F.3-5] when $\tau(r) \neq r$ we have

$$
\left(\alpha_{r}, \Theta\left(\alpha_{r}\right)\right)=0 \Leftrightarrow \mathfrak{g}_{2 \overline{\alpha_{r}}}=0,
$$

where $\bar{\alpha}$ is the restriction of $\alpha$ to $\mathfrak{a}_{\theta}=\{x \in i \mathfrak{t} \mid \theta(x)=-x\}$ and $\mathfrak{g}_{\psi}$ for $\psi \in \mathfrak{a}^{*}$ is the corresponding $\mathfrak{a}_{\theta}$-eigenspace. In terms of the Satake diagram, this means $r \in I_{\mathcal{C}}$ if and only if $\tau(r) \neq r$ and there is at least one white vertex on the path between $r$ and $\tau(r)$.

Let us further write

$$
I_{\mathrm{ns}}=\left\{r \in I \backslash X \mid \tau(r)=r \text { and }\left(\alpha_{r}, \alpha_{s}\right)=0 \text { for all } s \in X\right\},
$$


which correspond to the white vertices not connected to arrows or any black vertices in the Satake diagram. Next, we define

$$
I_{\mathcal{S}}=\left\{r \in I_{\mathrm{ns}} \mid a_{s r} \in 2 \mathbb{Z} \text { for all } s \in I_{\mathrm{ns}}\right\} \bigcup^{8}
$$

In other words, $I_{\mathcal{S}}$ consists of vertices $r \in I_{\mathrm{ns}}$ such that, when $s \in I_{\mathrm{ns}}$ is different from $r$, either there is no edge between $r$ and $s$ or there is an arrow of multiplicity two from $r$ to $s$ (when $\mathfrak{g}$ is simple, the latter possibility happens only for the type CI case corresponding to $\left.\mathfrak{k}^{\mathbb{C}} \cong \mathfrak{g l}_{l}(\mathbb{C}) \subset \mathfrak{s p}_{2 l}(\mathbb{C}) \cong \mathfrak{g}\right)$. Then we define

$$
\mathcal{S}_{\mathbf{q}}=\left\{\left(s_{r}(\mathbf{q})\right)_{r \in I \backslash X} \mid s_{r}(\mathbf{q}) \in \mathbb{C}\left(\mathbf{q}^{1 / d}\right) \text { and } s_{r}(\mathbf{q})=0 \text { for } r \notin I_{\mathcal{S}}\right\} .
$$

We will also use the shorthand notation

$$
\mathcal{T}_{\mathbf{q}}=\mathcal{C}_{\mathbf{q}} \times \mathcal{S}_{\mathbf{q}} \subseteq \mathbb{C}\left(\mathbf{q}^{1 / d}\right)^{I \backslash X} \times \mathbb{C}\left(\mathbf{q}^{1 / d}\right)^{I \backslash X},
$$

and write $\mathbf{t}=(\mathbf{c}, \mathbf{s})$ for a typical element in $\mathcal{T}_{\mathbf{q}}$.

Let $\mathfrak{g}_{X}$ be the Lie algebra generated by the elements $e_{r}, f_{r}, h_{r}$ with $r \in X$, and let $U_{\mathbf{q}}\left(\mathfrak{g}_{X}\right)$ be the $\mathbb{C}\left(\mathbf{q}^{1 / d}\right)$-algebra generated by the elements $E_{r}, F_{r}, K_{r}$ with $r \in X$. Moreover, let $U_{\mathbf{q}}\left(\mathfrak{h}^{\theta}\right)$ denote the algebra generated by the elements $K_{\omega}$ with $\Theta(\omega)=\omega$.

Definition 3.10. For $\mathbf{t}=(\mathbf{c}, \mathbf{s}) \in \mathcal{T}_{\mathbf{q}}$, we define $U_{\mathbf{q}}^{\mathbf{t}}\left(\mathfrak{k}^{\mathbb{C}}\right)=B_{\mathbf{c}, \mathbf{s}}$ to be the algebra generated by $U_{\mathbf{q}}\left(\mathfrak{g}_{X}\right)$, $U_{\mathbf{q}}\left(\mathfrak{h}^{\theta}\right)$ and the elements

$$
B_{r}=F_{r}+c_{r} \theta_{\mathbf{q}}\left(F_{r} K_{r}\right) K_{r}^{-1}+s_{r} K_{r}^{-1}, \quad r \in I \backslash X
$$

From Kol14, Proposition 5.2], it follows that $U_{\mathbf{q}}^{\mathbf{t}}\left(\mathfrak{k}^{\mathbb{C}}\right)$ is a right coideal subalgebra of $U_{\mathbf{q}}(\mathfrak{g})$. Again, the same constructions apply verbatim to the Hopf algebra $U_{q}(\mathfrak{g})$ for $q \neq 0$ not a root of unity, in which case $\mathcal{C}_{q}, \mathcal{S}_{q}$ are interpreted as subsets of $\mathbb{C}^{I \backslash X}$.

For $0<q<1$ and $U_{q}(\mathfrak{u})$ as before, it has been observed in [Let02, discussion before Theorem 7.6] that $U_{q}^{\mathbf{t}}\left(\mathfrak{k}^{\mathbb{C}}\right)$ should always be 'twisted' $*$-invariant, see also the discussion in Let00, Section 3]. For us, it will be important to know when $U_{q}^{\mathbf{t}}\left(\mathfrak{k}^{\mathbb{C}}\right)$ is genuinely $*$-invariant. We therefore refine the corresponding statement of Let02 in the theorem below. We thank Weiqiang Wang for pointing out to us that BW18, Proposition 4.6] considers a similar treatment of $*$-invariance. As however we are using different conventions and normalizations, we spell out some of the details.

Choose a set $I^{*}$ of representatives for $\tau$-orbits in $I \backslash X$. Note that by the proof of Kol14, Proposition 9.2], we can conjugate $U_{q}^{\mathbf{t}}\left(\mathfrak{k}^{\mathbb{C}}\right)$ with a unitary element in the (completed) Cartan part as to have $c_{r}>0$ when $\tau(r)=r$ or $r \in I_{\mathcal{C}}$ or $r \notin I^{*}$, without altering the invariance under $*$. We will hence assume this as an extra condition in the following ${ }^{9}$

Theorem 3.11. The algebra $U_{q}^{\mathbf{t}}\left(\mathfrak{k}^{\mathbb{C}}\right)$ is $*$-invariant if $s_{r} \in i \mathbb{R}$ and

$$
c_{\tau(r)} c_{r}=q^{\left(\Theta\left(\alpha_{r}\right)-\alpha_{r}, \alpha_{\tau(r)}\right)}
$$

for all $r \in I \backslash X$.

We will prove this theorem in Appendix B. Note that this implies that $c_{r}>0$ for all $r$ under the above convention on $c_{r}$.

In the following, we will write

$$
\mathcal{C}_{q, c}=\left\{\mathbf{c} \in \mathcal{C}_{q} \mid c_{r}>0, c_{\tau(r)} c_{r}=q^{\left(\Theta\left(\alpha_{r}\right)-\alpha_{\tau(r)}, \alpha_{r}\right)}\right\}, \quad \mathcal{S}_{q, c}=\left\{\mathbf{s} \in \mathcal{S}_{q} \mid s_{r} \in i \mathbb{R}\right\}, \quad \mathcal{T}_{q, c}=\mathcal{C}_{q, c} \times \mathcal{S}_{q, c}
$$

We then write $U_{q}^{\mathbf{t}}(\mathfrak{k})$ for the corresponding $*$-invariant coideal subalgebra of $U_{q}(\mathfrak{u})$. When $s_{r}=0$ and $0<c_{r}=c_{\tau(r)}$ satisfies (3.9) for all $r$ (fixing all $c_{r}$ ), we will refer to this as the no-parameter-case, and write $\mathbf{t}=(\mathbf{c}, \mathbf{s})=0$ and

$$
U_{q}^{\theta}(\mathfrak{k})=U_{q}^{0}(\mathfrak{k}) .
$$

Definition 3.12. By a finite admissible $*$-representation of $U_{q}^{\mathbf{t}}(\mathfrak{k})$ we mean any $*$-representation $(V, \pi)$ of $U_{q}^{\mathbf{t}}(\mathfrak{k})$ on a finite-dimensional pre-Hilbert space $V$ such that the operators $\pi\left(K_{\omega}\right)$ for $K_{\omega} \in U_{q}\left(\mathfrak{h}^{\theta}\right)$ have positive spectrum.

The following is now obvious.

\footnotetext{
${ }^{8}$ This corrects a misprint in the definition of the set $\mathcal{S}$ in Kol14 (5.11)], where the $a_{i j}$ should read $a_{j i}$.

${ }^{9}$ Although the claim for $r \notin I^{*}$ is not substantiated in Kol14 Proposition 9.2] a proof is easily provided, cf. Let02. Section 7, Variation 1].
} 
Theorem 3.13. Let $\operatorname{Rep}_{q}(\mathfrak{u})$ be the tensor $C^{*}$-category of finite admissible $*$-representations of $U_{q}(\mathfrak{u})$. Let $\operatorname{Rep}_{q}^{\mathbf{t}(\mathfrak{k})}$ be the $C^{*}$-category of finite admissible *-representations of $U_{q}^{\mathbf{t}}(\mathfrak{k})$. Then $\operatorname{Rep}_{q}^{\mathbf{t}}(\mathfrak{k})$ is a (strict) module $C^{*}$-category over $\operatorname{Rep}_{q}(\mathfrak{u})$ by setting $(V, \pi) \odot\left(V^{\prime}, \pi^{\prime}\right)$ (or $V \odot V^{\prime}$ for short) to be the representation of $U_{q}^{\mathbf{t}}(\mathfrak{k})$ on $V \otimes V^{\prime}$ with the module structure

$$
\pi \odot \pi^{\prime}=\left(\pi \otimes \pi^{\prime}\right) \circ \Delta_{\mid U_{q}^{\mathrm{t}}(\mathfrak{k})} .
$$

We write $\operatorname{Rep}_{q}^{\theta}(\mathfrak{k})=\operatorname{Rep}_{q}^{0}(\mathfrak{k})$ for the no-parameter case.

As the next theorem shows, the value of $\mathbf{t}$ is, in fact, irrelevant.

Theorem 3.14. The $\operatorname{Rep}_{q}(\mathfrak{u})$-module $C^{*}$-categories $\operatorname{Rep}_{q}^{\mathbf{t}}(\mathfrak{k})$ are all equivalent.

Proof. We will show in Appendix $\mathrm{C}$ that for $\mathbf{t}, \mathbf{t}^{\prime} \in \mathcal{T}_{q, c}$ there exist ${ }^{*}$-isomorphisms

$$
\pi_{\mathbf{t}, \mathbf{t}^{\prime}}: U_{q}^{\mathbf{t}}(\mathfrak{k}) \rightarrow U_{q}^{\mathbf{t}^{\prime}}(\mathfrak{k}) \text { such that }\left(\pi_{\mathbf{t}, \mathbf{t}^{\prime}} \otimes \mathrm{id}\right) \Delta=\Delta \pi_{\mathbf{t}, \mathbf{t}^{\prime}}
$$

on $U_{q}^{\mathbf{t}}(\mathfrak{k})$, and such that $\pi_{\mathbf{t}, \mathbf{t}^{\prime}}\left(K_{\omega}\right)$ is a positive scalar multiple of $K_{\omega}$ for each $K_{\omega} \in U_{q}\left(\mathfrak{h}^{\theta}\right)$. This clearly implies the theorem.

Hence in the following we can restrict to the no-parameter case. In order to turn $\operatorname{Rep}_{q}^{\theta}(\mathfrak{k})$ into a ribbon (twist-)braided module category, we will make use of the results from [BK15b, Kol17]. We again use freely the notation used there, which is compatible with the notation from [Kol14].

There is a slight adaption to be made as the results of [Kol17] require some extra assumptions on $\mathbf{c}, \mathbf{s}$ which are incompatible with the assumptions needed for our setting. To resolve this, let us for the moment return to the case of a formal variable q. In [Kol17, (3.13), (3.15) and (3.20)], it is assumed that $\mathbf{c} \in \mathcal{C}_{\mathbf{q}}$ and $\mathbf{s} \in \mathcal{S}_{\mathbf{q}}$ satisfy the extra relations

$$
c_{\tau(r)}(\mathbf{q})=\mathbf{q}^{\left(\alpha_{r}, \Theta\left(\alpha_{r}\right)-2 \rho_{X}\right)} c_{r}\left(\mathbf{q}^{-1}\right), \quad c_{\tau(r)}(\mathbf{q})=c_{\tau_{0}(r)}(\mathbf{q}), \quad s_{r}(\mathbf{q})=s_{r}\left(\mathbf{q}^{-1}\right)
$$

where $\tau_{0}$ is the diagram automorphism (nontrivial only for the $\mathrm{A}_{n}, \mathrm{D}_{2 n+1}$, and $\mathrm{E}_{6}$ types) characterized by $\alpha_{\tau_{0}(r)}=-w_{0} \alpha_{r}$ with $w_{0}$ the longest element in the Weyl group of $\mathfrak{g}$. As a case-by-case investigation of the Satake diagrams shows, the set $X$ is always preserved by $\tau_{0}$, and $\tau_{0}$ and $\tau$ commute BK15b Remark 7.2]. Upon adding roots of $\mathbf{q}$ if necessary, it follows that the special solution

$$
c_{r}^{\prime}(\mathbf{q})=\mathbf{q}^{\frac{1}{2}\left(\alpha_{r}, \Theta\left(\alpha_{r}\right)-2 \rho_{X}\right)}, \quad s_{r}^{\prime}(\mathbf{q})=0, \quad \mathbf{t}^{\prime}=\left(\mathbf{c}^{\prime}, \mathbf{s}^{\prime}\right)
$$

always satisfies the extra relations. Let now $\omega_{0} \in \mathfrak{h}^{*}$ be such that

$$
\left(\omega_{0}, \alpha_{r}\right)=0, \quad r \in X, \quad\left(\omega_{0}, \alpha_{r}\right)=\frac{1}{4}\left(\Theta\left(\alpha_{\tau(r)}\right)-\alpha_{\tau(r)}-\Theta\left(\alpha_{r}\right)+2 \rho_{X}, \alpha_{r}\right), \quad r \in I \backslash X .
$$

Since $\tau \omega_{0}$ satisfies the same conditions, $\omega_{0}$ is $\tau$-invariant. Moreover, since $\omega_{0}$ vanishes on the roots in $X$, it follows that $\Theta\left(\omega_{0}\right)=-\tau\left(\omega_{0}\right)=-\omega_{0}$, so

$$
\left(\omega_{0}, \alpha_{r}-\Theta\left(\alpha_{r}\right)\right)=2\left(\omega_{0}, \alpha_{r}\right)=\frac{1}{2}\left(\Theta\left(\alpha_{\tau(r)}\right)-\alpha_{\tau(r)}-\Theta\left(\alpha_{r}\right)+2 \rho_{X}, \alpha_{r}\right) \quad r \in I \backslash X .
$$

Let $\gamma$ be the Hopf algebra automorphism $\operatorname{Ad}\left(K_{\omega_{0}}\right)$ of $U_{\mathbf{q}}(\mathfrak{g})$, which is characterized by

$$
E_{r} \mapsto \mathbf{q}^{\left(\omega_{0}, \alpha_{r}\right)} E_{r}, \quad F_{r} \mapsto \mathbf{q}^{-\left(\omega_{0}, \alpha_{r}\right)} F_{r}, \quad K_{\omega} \mapsto K_{\omega} .
$$

Then, using

$$
\operatorname{Ad}\left(K_{\omega_{0}}\right)\left(F_{r}+c_{r}^{\prime} \theta_{\mathbf{q}}\left(F_{r} K_{r}\right) K_{r}^{-1}\right)=\mathbf{q}^{-\left(\omega_{0}, \alpha_{r}\right)}\left(F_{r}+\mathbf{q}^{\left(\omega_{0}, \alpha_{r}-\Theta\left(\alpha_{r}\right)\right)} c_{r}^{\prime} \theta_{\mathbf{q}}\left(F_{r} K_{r}\right) K_{r}^{-1}\right),
$$

we obtain

$$
\gamma\left(U_{\mathbf{q}}^{\mathbf{t}^{\prime}}\left(\mathfrak{k}^{\mathbb{C}}\right)\right)=U_{\mathbf{q}}^{\theta}\left(\mathfrak{k}^{\mathbb{C}}\right)
$$

where we interpret our non-parameter case in the setting of the indeterminate variable $\mathbf{q}$.

Denote by $\mathscr{U}_{\mathbf{q}}(\mathfrak{g})=\bigoplus_{\varpi \in P^{+}} \operatorname{End}_{\mathbb{C}(\mathbf{q})}\left(V_{\varpi}^{\mathbf{q}}\right)$ the discrete multiplier bi-algebra over $\mathbb{C}(\mathbf{q})$ associated to $U_{\mathbf{q}}(\mathfrak{g})$, so in particular $U_{\mathbf{q}}(\mathfrak{g}) \subseteq M\left(\mathscr{U}_{\mathbf{q}}(\mathfrak{g})\right)=\prod_{\varpi \in P^{+}} \operatorname{End}_{\mathbb{C}(\mathbf{q})}\left(V_{\varpi}^{\mathbf{q}}\right)$. Let

$$
\mathscr{K}^{\prime} \in U_{\mathbf{q}}^{\mathbf{t}^{\prime}}\left(\mathfrak{k}^{\mathbb{C}}\right) \hat{\otimes} U_{\mathbf{q}}(\mathfrak{g})
$$

be the universal $K$-matrix for $U_{q}^{\mathbf{t}^{\prime}\left(\mathfrak{k}^{\mathbb{C}}\right)}$ BK15b Kol17, Theorem 3.11], and write

$$
\widetilde{\mathscr{K}}=(\gamma \otimes \gamma)\left(\mathscr{K}^{\prime}\right) \in U_{\mathbf{q}}^{\theta}\left(\mathfrak{k}^{\mathbb{C}}\right) \hat{\otimes} U_{\mathbf{q}}(\mathfrak{g})
$$

Then by $\tau \tau_{0}$-invariance of $\omega_{0}$,

$$
\widetilde{\mathscr{K}} \Delta(b)=\left(\operatorname{id} \otimes \tau \tau_{0}\right)(\Delta(b)) \widetilde{\mathscr{K}}, \quad b \in U_{\mathbf{q}}^{\theta}\left(\mathfrak{k}^{\mathbb{C}}\right) .
$$


Moreover, since the $R$-matrix $\mathscr{R} \in U_{\mathbf{q}}(\mathfrak{g}) \hat{\otimes} U_{\mathbf{q}}(\mathfrak{g})$ is invariant under $\gamma \otimes \gamma$, we also retain

$$
(\Delta \otimes \mathrm{id})(\widetilde{\mathscr{K}})=\left(\mathrm{id} \otimes \tau \tau_{0} \otimes \mathrm{id}\right)\left(\mathscr{R}_{32}\right) \widetilde{\mathscr{K}}_{13} \mathscr{R}_{23}, \quad(\mathrm{id} \otimes \Delta)(\widetilde{\mathscr{K}})=\mathscr{R}_{32} \widetilde{\mathscr{K}}_{13}\left(\mathrm{id} \otimes \tau \tau_{0} \otimes \mathrm{id}\right)\left(\mathscr{R}_{23}\right) \widetilde{\mathscr{K}}_{12} .
$$

All of the above can be performed as well in the setting of $0<q<1$ a real number, and in particular (3.10), 3.11) and $(3.12$ continue to hold. (For example, all constructions, including those involving the bar involution BK15a, Kol17], can be performed over the $\mathbb{C}$-algebra $\mathbb{C}[\mathbf{q}] /\left(\mathbf{q}^{2}-\left(q+q^{-1}\right) \mathbf{q}+1\right)$, from which one can specialize to $\mathbf{q}=q$.) Let

$$
\mathscr{K}=\left(\operatorname{id} \otimes \tau \tau_{0}\right)(\widetilde{\mathscr{K}}) \in U_{q}^{\theta}(\mathfrak{k}) \hat{\otimes} U_{q}(\mathfrak{u}) .
$$

Then (3.11) becomes

$$
\mathscr{K}\left(\left(\operatorname{id} \otimes \tau \tau_{0}\right)(\Delta(b))=\Delta(b) \mathscr{K}, \quad \forall b \in U_{q}^{\theta}(\mathfrak{k}) .\right.
$$

We also have $\left(\tau \tau_{0} \otimes \tau \tau_{0}\right)(\mathscr{R})=\mathscr{R}$, since any automorphism of the Dynkin diagram will leave the universal $R$-matrix invariant, see CP95, Corollary 8.3.12] (or, just to check this equality in the multiplier algebra one may use the fact that $\mathscr{R}$ is determined by its action on the tensor product of lowest and highest weight vectors). It follows that

$$
(\Delta \otimes \mathrm{id})(\mathscr{K})=\mathscr{R}_{32} \mathscr{K}_{13}\left(\left(\mathrm{id} \otimes \tau \tau_{0}\right)(\mathscr{R})\right)_{23}, \quad(\operatorname{id} \otimes \Delta)(\mathscr{K})=(\operatorname{id} \otimes \Delta)(\mathscr{K}) \mathscr{K}_{12} .
$$

We hence obtain the following theorem.

Theorem 3.15. With respect to the braiding

$$
\eta_{X, U}=\left(\pi_{X} \otimes \pi_{U}\right)(\mathscr{K}),
$$

$\operatorname{Rep}_{q}^{\theta}(\mathfrak{k})$ is a (strict) ribbon $\tau \tau_{0}$-braided module $C^{*}$-category over the braided tensor $C^{*}$-category $\operatorname{Rep}_{q}(\mathfrak{u})$, where the latter is equipped with the braiding coming from the universal $R$-matrix $\mathscr{R}$.

3.4. Ribbon braided module $\mathbf{C}^{*}$-categories from quantum group automorphisms. Fix again a compact semisimple Lie algebra $\mathfrak{u}$ with Chevalley generators $\left\{e_{r}, f_{r}, h_{r} \mid r \in I\right\}$ in $\mathfrak{g}=\mathfrak{u}^{\mathbb{C}}$, and let $\mathfrak{h}=\mathfrak{t} \oplus \mathfrak{a}$ be the complex Cartan subalgebra with vector part $\mathfrak{a}=\oplus_{r} \mathbb{R} h_{r}$ and compact part $\mathfrak{t}=i \mathfrak{a}$.

Let $(Y, \mu)$ be a Vogan diagram with associated involution $\nu=\nu(Y, \mu)$. For $0<q<1$, we then obtain an involutive Hopf $*$-algebra automorphism $\nu_{q}$ of $U_{q}(\mathfrak{u})$ by

$$
\nu_{q}\left(E_{r}\right)=\epsilon_{r} E_{\mu(r)}, \quad \nu_{q}\left(F_{r}\right)=\epsilon_{r} F_{\mu(r)}, \quad \nu_{q}\left(K_{\alpha}\right)=K_{N(\alpha)},
$$

where $N$ denotes the involutive transformation dual to $\nu_{\mid \mathfrak{h}}$. Note that $\nu_{q}$ also preserves the Borel subalgebras $U_{q}\left(\mathfrak{b}^{ \pm}\right)$. In the following, it will be convenient to work for a while with versions of $U_{q}(\mathfrak{u})$ and $U_{q}(\mathfrak{g})$ with an extra Cartan part added.

Consider independent copies of the Hopf algebras $U_{q}\left(\mathfrak{b}^{ \pm}\right)=\left(U_{q}\left(\mathfrak{b}^{ \pm}\right), \Delta, \varepsilon, S\right)$ with generators now denoted by $X_{r}^{ \pm}, L_{\omega}^{ \pm}$, so in particular

$$
L_{\omega}^{ \pm} X_{r}^{ \pm}=q^{ \pm\left(\omega, \alpha_{r}\right)} X_{r}^{ \pm} L_{\omega}^{ \pm}, \quad \Delta\left(X_{r}^{+}\right)=X_{r}^{+} \otimes 1+L_{r}^{+} \otimes X_{r}^{+}, \quad \Delta\left(X_{r}^{-}\right)=X_{r}^{-} \otimes\left(L_{r}^{-}\right)^{-1}+1 \otimes X_{r}^{-} .
$$

Consider the skew-pairing between $U_{q}\left(\mathfrak{b}^{-}\right)$and $U_{q}\left(\mathfrak{b}^{+}\right)$characterized by

$$
\left(X_{r}^{-}, X_{s}^{+}\right)=\delta_{r s}\left(q_{r}^{-1}-q_{r}\right)^{-1}, \quad\left(L_{\alpha}^{-}, L_{\beta}^{+}\right)=q^{-(\alpha, \beta)},
$$

and being zero between the other generators. Here the skew-pairing property means we have

$$
(X Y, Z)=(X \otimes Y, \Delta(Z)), \quad(X, Y Z)=\left(\Delta^{\mathrm{op}}(X), Y \otimes Z\right) .
$$

We can then make a new skew-pairing by twisting with $\nu_{q}$,

$$
(X, Y)_{\nu}=\left(\nu_{q}(X), Y\right) \text {. }
$$

Write $(X, Y)_{0}=(X, Y)$ and $(X, Y)_{1}=(X, Y)_{\nu}$. Define for $k, l \in\{0,1\}$ the unital algebra $U_{q}^{k l}(\mathfrak{g} \oplus \mathfrak{h})$ generated by the algebras $U_{q}\left(\mathfrak{b}^{ \pm}\right)$with interchange relation

$$
Y X=\left(X_{(1)}, Y_{(1)}\right)_{k} X_{(2)} Y_{(2)}\left(S\left(X_{(3)}\right), Y_{(3)}\right)_{l}, \quad Y \in U_{q}\left(\mathfrak{b}^{+}\right), X \in U_{q}\left(\mathfrak{b}^{-}\right) .
$$

Then it is easily verified that we have as universal relations between the generators those of $U_{q}\left(\mathfrak{b}^{ \pm}\right)$ together with

$$
\begin{gathered}
L_{\omega}^{+} L_{\chi}^{-}=q^{\left(\omega, N^{l}(\chi)-N^{k}(\chi)\right)} L_{\chi}^{-} L_{\omega}^{+}, \\
L_{\omega}^{-} X_{r}^{+}=q^{\left(\alpha_{r}, N^{k}(\omega)\right)} X_{r}^{+} L_{\omega}^{-}, \quad L_{\omega}^{+} X_{r}^{-}=q^{-\left(\alpha_{r}, N^{l}(\omega)\right)} X_{r}^{-} L_{\omega}^{+}, \\
{\left[X_{r}^{+}, X_{s}^{-}\right]=\frac{\delta_{r, \mu^{l}(s)} \epsilon_{s}^{l} L_{r}^{+}-\delta_{r, \mu^{k}(s)} \epsilon_{s}^{k}\left(L_{s}^{-}\right)^{-1}}{q_{r}-q_{r}^{-1}}}
\end{gathered}
$$


and that moreover there are no 'extra relations' in the sense that the multiplication map gives isomorphisms

$$
U_{q}\left(\mathfrak{b}^{+}\right) \otimes U_{q}\left(\mathfrak{b}^{-}\right) \cong U_{q}^{k l}(\mathfrak{g} \oplus \mathfrak{h}) \cong U_{q}\left(\mathfrak{b}^{-}\right) \otimes U_{q}\left(\mathfrak{b}^{+}\right) .
$$

We can endow $U_{q}^{k l}(\mathfrak{g} \oplus \mathfrak{h})$ with the unique $*$-structure such that

$$
\left(X_{r}^{+}\right)^{*}=X_{r}^{-} L_{r}^{-}, \quad\left(L_{r}^{+}\right)^{*}=L_{r}^{-},
$$

and we denote this $*$-algebra by $U_{q}^{k l}(\mathfrak{u} \oplus \mathfrak{a})$. The $*$-algebra $U_{q}^{00}(\mathfrak{u} \oplus \mathfrak{a})$ is known to be a cover of the Hopf *-algebra $U_{q}(\mathfrak{u})$ under

$$
X_{r}^{+} \mapsto E_{r}, \quad X_{r}^{-} \mapsto F_{r}, \quad L_{\alpha}^{ \pm} \mapsto K_{\alpha} .
$$

It is also immediate from the construction that the family $\left(U_{q}^{k l}(\mathfrak{u} \oplus \mathfrak{a})\right)_{k, l}$ form a Hopf-Galois system in the sense of Bic14, Definition 2.4] with $*$-preserving comultiplications

$$
\Delta=\Delta_{k l}^{m}: U_{q}^{k l}(\mathfrak{u} \oplus \mathfrak{a}) \rightarrow U_{q}^{k m}(\mathfrak{u} \oplus \mathfrak{a}) \otimes U_{q}^{m l}(\mathfrak{u} \oplus \mathfrak{a})
$$

given by the usual comultiplication on $U_{q}\left(\mathfrak{b}^{ \pm}\right)$, and with the antipodes

$$
S=S^{k l}: U_{q}^{k l}(\mathfrak{u} \oplus \mathfrak{a}) \rightarrow U_{q}^{l k}(\mathfrak{u} \oplus \mathfrak{a})
$$

determined by the ordinary antipodes on $U_{q}\left(\mathfrak{b}^{ \pm}\right)$. In the following, we denote by

$$
\widetilde{\alpha}: U_{q}^{10}(\mathfrak{u} \oplus \mathfrak{a}) \rightarrow U_{q}^{10}(\mathfrak{u} \oplus \mathfrak{a}) \otimes U_{q}(\mathfrak{u})
$$

the composition of $\Delta_{10}^{0}$ with the projection $U_{q}^{00}(\mathfrak{u} \oplus \mathfrak{a}) \rightarrow U_{q}(\mathfrak{u})$.

Let $U_{q}^{10}\left(\mathfrak{u}_{\mathrm{r}} \oplus \mathfrak{a}\right)$ be the $*$-subalgebra of $U_{q}^{10}(\mathfrak{u} \oplus \mathfrak{a})$ generated by

$$
X_{r}^{-}, \quad\left(X_{r}^{-}\right)^{*} \quad(r \in I), \quad L_{\omega}^{ \pm} \quad(\omega \in P, N(\omega)=\omega), \quad L_{\omega}^{+} L_{-\omega}^{-} \quad(\omega \in P) .
$$

Then $U_{q}^{10}\left(\mathfrak{u}_{\mathrm{r}} \oplus \mathfrak{a}\right)$ is precisely the $*$-algebra of elements commuting with all $L_{\omega}^{+} L_{-\omega}^{-}$, and is stable under the coaction $\widetilde{\alpha}$ of $U_{q}(\mathfrak{u})$. Moreover, the ensuing coaction of $U_{q}(\mathfrak{u})$ has $L_{\omega}^{+} L_{-\omega}^{-}$in its space of coinvariants. Let us write $U_{q}^{10}\left(\mathfrak{u}_{\mathrm{r}}\right)$ for the $*$-algebra obtained by taking the quotient of $U_{q}^{10}\left(\mathfrak{u}_{\mathrm{r}} \oplus \mathfrak{a}\right)$ with the ideal generated by the $1-L_{\omega}^{+} L_{-\omega}^{-}$. Then we obtain a coaction $\alpha$ of $U_{q}(\mathfrak{u})$ on $U_{q}^{10}\left(\mathfrak{u}_{\mathrm{r}}\right)$. It is, in fact, not hard to verify that $U_{q}^{10}\left(\mathfrak{u}_{\mathrm{r}}\right)$ is the universal $*$-algebra generated (as a $*$-algebra) by the elements $F_{r}$ for $r \in I$ and the selfadjoint elements $K_{\omega}$ for $\omega \in P^{N}$ (obtained as images of $X_{r}^{-}$and $L_{\omega}^{+}$respectively) such that $K_{\omega}, F_{r}$ satisfy the relations of $U_{q}\left(\mathfrak{b}^{-}\right)$and such that

$$
F_{r}^{*} F_{s}-q^{\left(\alpha_{r}, \alpha_{s}\right)} F_{s} F_{r}^{*}=\frac{\delta_{r, s}-\delta_{r, \mu(s)} \epsilon_{s} q^{-\frac{1}{2}\left(\alpha_{r}, \alpha_{s}-\alpha_{\mu(r)}\right)} K_{\alpha_{\mu(r)}+\alpha_{r}}^{-1}}{q_{r}-q_{r}^{-1}},
$$

the coaction $\alpha$ by $U_{q}(\mathfrak{u})$ being given by the ordinary formulas. Note that if $\mathfrak{k} \subseteq \mathfrak{u}$ is of equal rank (i.e., $\nu$ acts trivially on $\mathfrak{h}$ ), we can identify $U_{q}^{10}\left(\mathfrak{u}_{\mathrm{r}}\right)$ with $U_{q}(\mathfrak{u})$ as a vector space by the natural triangular decomposition. We will denote the *-algebra generated by the elements $K_{\omega}$ with $N(\omega)=\omega$ by $U_{q}\left(\mathfrak{t}^{\nu}\right)$.

Next let us review the representation theory of $U_{q}^{10}\left(\mathfrak{u}_{\mathrm{r}}\right)$, cf. [DC13.

Definition 3.16. We call (resp. finite) admissible $U_{q}^{10}\left(\mathfrak{u}_{\mathrm{r}}\right)$-module any (resp. finitely generated) left $U_{q}^{10}\left(\mathfrak{u}_{\mathrm{r}}\right)$-module for which $U_{q}\left(\mathfrak{n}^{-}\right)$acts locally finitely and for which the $K_{\omega} \in U_{q}\left(\mathfrak{t}^{\nu}\right)$ act as semisimple transformations with positive eigenvalues. We call (resp. finite) admissible *-representation any (resp. finite) admissible module $M$ endowed with a pre-Hilbert space structure for which

$$
\langle v, X w\rangle=\left\langle X^{*} v, w\right\rangle, \quad\left(v, w \in M, X \in U_{q}^{10}\left(\mathfrak{u}_{\mathrm{r}}\right)\right) .
$$

Note that in general, the finite admissible $*$-representations will not be on finite-dimensional Hilbert spaces, even when they are irreducible.

For $\omega \in \mathfrak{a}^{\nu}$, we call a vector $\xi$ in a $U_{q}^{10}\left(\mathfrak{u}_{\mathrm{r}}\right)$-module a weight vector of weight $\omega$ if

$$
K_{\chi} \xi=q^{(\omega, \chi)} \xi .
$$

We call a vector $\eta$ in a $U_{q}^{10}\left(\mathfrak{u}_{\mathrm{r}}\right.$ )-module a lowest weight vector (of weight $\omega$ ) if it is a weight vector (of weight $\omega$ ) vanishing under the action of the $F_{r}$. We call a $U_{q}^{10}\left(\mathfrak{u}_{\mathrm{r}}\right)$-module a lowest weight-module (of weight $\omega$ ) if it is generated by a lowest weight vector (of weight $\omega$ ).

Lemma 3.17. Any finite admissible *-representation of $U_{q}^{10}\left(\mathfrak{u}_{\mathrm{r}}\right)$ is a finite direct sum of irreducible admissible *-representations. Moreover, an admissible *-representation is lowest weight if and only if it is irreducible, and in this case the space of its self-intertwiners consists of the scalars. 
Proof. In a finite admissible *-representation the eigenspaces of $K_{2 \rho}$, where $\rho$ is the ( $N$-invariant) halfsum of the positive roots, must be finite-dimensional. Since any subrepresentation of a finite admissible *-representation is again finite admissible, any admissible $*$-representation is a direct sum of a finite number of irreducible admissible $*$-representations as we can take orthogonal complements in each $K_{2 \rho^{-}}$ eigenspace.

If now $M$ is admissible and irreducible, we can obtain a lowest weight vector by repeatedly applying $F_{r}$ 's to a weight vector. By irreducibility, the module spanned by the lowest weight vector must be $M$. Conversely, if $M$ is admissible and of lowest weight $\omega_{0}$, the space of $\omega_{0}$-weight vectors must be onedimensional and can hence be contained in only one irreducible component.

The final statement is immediate from Schur's lemma.

Lemma 3.18. A lowest weight *-representation $M$ is completely determined up to isomorphism by its lowest weight.

Proof. Let $\omega_{0}$ be the lowest weight. By inducing from a one-dimensional module for the Borel part, we can turn $U_{q}\left(\mathfrak{n}^{-}\right)^{*}$ into a $U_{q}^{10}\left(\mathfrak{u}_{\mathrm{r}}\right)$-module with 1 as a lowest weight vector at weight $\omega_{0}$. This module has an invariant sesqui-linear form by the triangular decomposition. By multiplying with a scalar if necessary, the natural map $U_{q}\left(\mathfrak{n}^{-}\right)^{*} \rightarrow M$ must then preserve this form, again by the triangular decomposition. Hence the kernel of the above map must lie in the kernel of the form on $U_{q}\left(\mathfrak{n}^{-}\right)^{*}$, and must then be equal to this kernel. This completely determines $M$.

In the following, we say that a $\nu$-invariant weight $\omega_{0}$ is $U_{q}^{10}\left(\mathfrak{u}_{\mathrm{r}}\right)$-adapted if it arises as the lowest weight in a lowest weight admissible $*$-representation, and we write the associated lowest weight $*$-representation as $M_{\omega_{0}}$. The modules $M_{\omega_{0}}$, with $\omega_{0}$ ranging over the $U_{q}^{10}\left(\mathfrak{u}_{\mathrm{r}}\right)$-adapted weights, then form a maximal collection of pairwise non-isomorphic irreducible admissible $U_{q}^{10}\left(\mathfrak{u}_{\mathrm{r}}\right)$-representations.

Definition 3.19. We write $\operatorname{Rep}_{q}^{\nu}\left(\mathfrak{u}^{\nu}\right)$ for the $\mathrm{C}^{*}$-category of finite admissible $*$-representations of $U_{q}^{10}\left(\mathfrak{u}_{\mathrm{r}}\right)$.

From the above discussion we see that the morphism spaces between finite admissible $*$-representations are finite-dimensional, and consist of adjointable maps. We now have the following result.

Theorem 3.20. If $M$ is a finite admissible *-representation of $U_{q}^{10}\left(\mathfrak{u}_{\mathrm{r}}\right)$, and $V$ a finite admissible representation of $U_{q}(\mathfrak{u})$, then $M \otimes V$ with the representation $\left(\pi_{M} \otimes \pi_{V}\right) \circ \alpha$ is a finite admissible *representation of $U_{q}^{10}\left(\mathfrak{u}_{\mathrm{r}}\right)$, making $\operatorname{Rep}_{q}^{\nu}\left(\mathfrak{u}^{\nu}\right)$ a module $C^{*}$-category over $\operatorname{Rep}_{q}(\mathfrak{u})$.

We now show that $\operatorname{Rep}_{q}^{\nu}\left(\mathfrak{u}^{\nu}\right)$ is a ribbon $\nu_{q}$-braided module $\mathrm{C}^{*}$-category, where $\nu_{q}$ also denotes the (strict) braided autoequivalence of $\operatorname{Rep}_{q}(\mathfrak{u})$ induced by the involution $\nu_{q}$ on $U_{q}(\mathfrak{u})$.

Let again

$$
\mathscr{R} \in U_{q}(\mathfrak{u}) \hat{\otimes} U_{q}(\mathfrak{u})=\prod_{\varpi, \chi} B\left(V_{\varpi}\right) \otimes B\left(V_{\chi}\right)
$$

be the universal $R$-matrix of $U_{q}(\mathfrak{u})$. We have $\left(\nu_{q} \otimes \nu_{q}\right)(\mathscr{R})=\mathscr{R}$, as can be seen from a concrete formula which contains $E_{r}$ and $F_{r}$ in pairs and is stable under diagram automorphism CP95, Section 8.3]. Recall also that it can be characterized by a pairing of $U_{q}\left(\mathfrak{b}^{-}\right)$and $U_{q}\left(\mathfrak{b}^{+}\right)$, in the sense that for $Y \in U_{q}\left(\mathfrak{b}^{-}\right)$, when $\omega$ is a functional on $U_{q}(\mathfrak{u})$ factoring through a finite admissible $*$-representation, one has

$$
Z=(\text { id } \otimes \omega)(\mathscr{R}) \in U_{q}\left(\mathfrak{b}^{+}\right) \quad \text { and } \quad(Y, Z)=\omega(Y) .
$$

Similarly, if $Z \in U_{q}\left(\mathfrak{b}^{+}\right)$we have then

$$
Y=(\omega \otimes \mathrm{id})(\mathscr{R}) \in U_{q}\left(\mathfrak{b}^{-}\right) \quad \text { and } \quad(Y, Z)=\omega(Z) .
$$

The first assertions in the above two lines will be written as

$$
\mathscr{R} \in U_{q}\left(\mathfrak{b}^{+}\right) \hat{\otimes} U_{q}\left(\mathfrak{b}^{-}\right) .
$$

We can then interpret $\mathscr{R}$ as an element

$$
\widetilde{\mathscr{R}} \in U_{q}^{10}(\mathfrak{u} \oplus \mathfrak{a}) \hat{\otimes} U_{q}\left(\mathfrak{b}^{-}\right) \subseteq U_{q}^{10}(\mathfrak{u} \oplus \mathfrak{a}) \hat{\otimes} U_{q}(\mathfrak{u}) .
$$

Similarly, $\mathscr{R}_{21}$ can be interpreted as an element $\widetilde{\mathscr{R}}_{21}$ in $U_{q}^{10}(\mathfrak{u} \oplus \mathfrak{a}) \hat{\otimes} U_{q}\left(\mathfrak{b}^{+}\right)$(strictly speaking we should write $\widetilde{\mathscr{R}_{21}}$, which is however a bit more awkward notation).

Lemma 3.21. For $X \in U_{q}\left(\mathfrak{b}^{+}\right)$and $Y \in U_{q}\left(\mathfrak{b}^{-}\right)$, we have the following identities in $U_{q}^{10}(\mathfrak{u} \oplus \mathfrak{a}) \hat{\otimes} U_{q}(\mathfrak{u})$ :

$$
\begin{aligned}
\widetilde{\mathscr{R}} \Delta(X) & =\Delta^{\mathrm{op}}(X) \widetilde{\mathscr{R}}, & \widetilde{\mathscr{R}} \Delta(Y) & =\left(\operatorname{id} \otimes \nu_{q}\right) \Delta^{\mathrm{op}}(Y) \widetilde{\mathscr{R}}, \\
\widetilde{\mathscr{R}}_{21}\left(\left(\mathrm{id} \otimes \nu_{q}\right) \Delta^{\mathrm{op}}(X)\right) & =\Delta(X) \widetilde{\mathscr{R}}_{21}, & \widetilde{\mathscr{R}}_{21} \Delta^{\mathrm{op}}(Y) & =\Delta(Y) \widetilde{\mathscr{R}}_{21} .
\end{aligned}
$$


Proof. Since the first legs of $\Delta(X)$ and $\widetilde{\mathscr{R}}$ both lie in $U_{q}\left(\mathfrak{b}^{+}\right)$, the first identity can be interpreted inside $U_{q}\left(\mathfrak{b}^{+}\right) \hat{\otimes} U_{q}(\mathfrak{g})$, and thus holds from the ordinary $R$-matrix relations. For the second identity, we take a functional $\omega$ on $U_{q}(\mathfrak{u})$ factorizing through a finite admissible $*$-representation. Then, we have

$$
(\operatorname{id} \otimes \omega)(\widetilde{\mathscr{R}}) Y=Y_{(2)}(\operatorname{id} \otimes \omega)\left(\left(1 \otimes \nu_{q}\left(Y_{(1)}\right)\right) \widetilde{\mathscr{R}}\left(1 \otimes S\left(Y_{(3)}\right)\right)\right),
$$

from 3.13 and the fact that $(Y,($ id $\otimes \omega)(\widetilde{R}))=\omega(Y)$, hence

$$
\widetilde{\mathscr{R}} \Delta(Y)=\left(Y_{(2)} \otimes \nu_{q}\left(Y_{(1)}\right)\right) \widetilde{\mathscr{R}}\left(1 \otimes S\left(Y_{(3)}\right) Y_{(4)}\right)=\left(\mathrm{id} \otimes \nu_{q}\right)\left(\Delta^{\mathrm{op}}(Y)\right) \widetilde{\mathscr{R}} .
$$

The remaining two identities are obtained in a similar way.

Let $v=e^{\pi i \hbar C}$ be the natural ribbon element, where $C$ is the quantum Casimir element in the center of $M\left(\mathscr{U}_{q}(\mathfrak{u})\right)$ acting on $V_{\varpi}^{q}$ by the same scalar as the classical Casimir element of $\mathfrak{u}$ on $V_{\varpi}$. Recall that

$$
\mathscr{R}_{21} \mathscr{R} \Delta(v)=v \otimes v \text {. }
$$

Definition 3.22. We define

$$
\widetilde{\mathscr{E}}=\widetilde{\mathscr{R}}_{21}\left(\mathrm{id} \otimes \nu_{q}\right)(\widetilde{\mathscr{R}})\left(1 \otimes v^{-1}\right) \in U_{q}^{10}(\mathfrak{u} \oplus \mathfrak{a}) \hat{\otimes} U_{q}(\mathfrak{u}) .
$$

Proposition 3.23. The element $\widetilde{\mathscr{E}}$ satisfies the following properties:

$$
\begin{gathered}
\widetilde{\mathscr{E}}\left(\mathrm{id} \otimes \nu_{q}\right)(\widetilde{\alpha}(x))=\widetilde{\alpha}(x) \widetilde{\mathscr{E}} \text { for all } x \in U_{q}^{10}(\mathfrak{u} \oplus \mathfrak{a}), \\
(\widetilde{\alpha} \otimes \mathrm{id})(\widetilde{\mathscr{E}})=\mathscr{R}_{32} \widetilde{\mathscr{E}}_{13}\left(\mathrm{id} \otimes \nu_{q}\right)(\mathscr{R})_{23}, \quad(\mathrm{id} \otimes \Delta) \widetilde{\mathscr{E}}=(\widetilde{\alpha} \otimes \mathrm{id})(\widetilde{\mathscr{E}}) \widetilde{\mathscr{E}}_{12}
\end{gathered}
$$

Proof. The first identity follows from Lemma 3.21 and that $\nu_{q}$ is involutive, by considering separately elements in $U_{q}\left(\mathfrak{b}^{+}\right)$and $U_{q}\left(\mathfrak{b}^{-}\right)$. The second identity is also immediate from the properties of the $R$ matrix.

Writing out the last equality and using the defining property of $v$, we see that it is equivalent to

$$
(\mathrm{id} \otimes \Delta)\left(\widetilde{\mathscr{R}}_{21}\left(\mathrm{id} \otimes \nu_{q}\right)(\widetilde{\mathscr{R}})\right) \mathscr{R}_{32} \mathscr{R}_{23}=\mathscr{R}_{32} \widetilde{\mathscr{R}}_{31}\left(\mathrm{id} \otimes \nu_{q}\right)(\widetilde{\mathscr{R}})_{13}\left(\mathrm{id} \otimes \nu_{q}\right)(\mathscr{R})_{23} \widetilde{\mathscr{R}}_{21}\left(\mathrm{id} \otimes \nu_{q}\right)(\widetilde{\mathscr{R}})_{12} \text {. }
$$

Noting

$$
\begin{aligned}
(\mathrm{id} \otimes \Delta)\left(\widetilde{\mathscr{R}}_{21}\left(\mathrm{id} \otimes \nu_{q}\right)(\widetilde{\mathscr{R}})\right) \mathscr{R}_{32} \mathscr{R}_{23} & =\mathscr{R}_{32}\left(\mathrm{id} \otimes \Delta^{\mathrm{op}}\right)\left(\widetilde{\mathscr{R}}_{21}\left(\mathrm{id} \otimes \nu_{q}\right)(\widetilde{\mathscr{R}})\right) \mathscr{R}_{23} \\
& =\mathscr{R}_{32} \widetilde{\mathscr{R}}_{31} \widetilde{\mathscr{R}}_{21}\left(\mathrm{id} \otimes \Delta^{\mathrm{op}}\right)\left(\left(\mathrm{id} \otimes \nu_{q}\right)(\widetilde{\mathscr{R}})\right) \mathscr{R}_{23} \\
& =\mathscr{R}_{32} \widetilde{\mathscr{R}}_{31} \widetilde{\mathscr{R}}_{21} \mathscr{R}_{23}(\mathrm{id} \otimes \Delta)\left(\left(\mathrm{id} \otimes \nu_{q}\right)(\widetilde{\mathscr{R}})\right) \\
& =\mathscr{R}_{32} \widetilde{\mathscr{R}}_{31} \widetilde{\mathscr{R}}_{21} \mathscr{\mathscr { R }}_{23}\left(\mathrm{id} \otimes \nu_{q}\right)(\widetilde{\mathscr{R}})_{13}\left(\mathrm{id} \otimes \nu_{q}\right)(\widetilde{\mathscr{R}})_{12},
\end{aligned}
$$

3.14 will follow from

$$
\left(\mathrm{id} \otimes \nu_{q}\right)(\widetilde{\mathscr{R}})_{13}\left(\mathrm{id} \otimes \nu_{q}\right)(\mathscr{R})_{23} \widetilde{\mathscr{R}}_{21}=\widetilde{\mathscr{R}}_{21} \mathscr{R}_{23}\left(\mathrm{id} \otimes \nu_{q}\right)(\widetilde{\mathscr{R}})_{13} .
$$

This is a consequence of the commutation relations in Lemma 3.21 .

$$
\begin{aligned}
\left(\mathrm{id} \otimes \nu_{q}\right)(\widetilde{\mathscr{R}})_{13}\left(\mathrm{id} \otimes \nu_{q}\right)(\mathscr{R})_{23} \widetilde{\mathscr{R}}_{21} & =(\Delta \otimes \mathrm{id})\left(\left(\mathrm{id} \otimes \nu_{q}\right) \widetilde{\mathscr{R}}\right) \widetilde{\mathscr{R}}_{21} \\
& =\widetilde{\mathscr{R}}_{21}\left(\mathrm{id} \otimes \nu_{q} \otimes \nu_{q}\right)\left(\Delta^{\mathrm{op}} \otimes \mathrm{id}\right)(\widetilde{\mathscr{R}}) \\
& =\widetilde{\mathscr{R}}_{21} \mathscr{R}_{23}\left(\mathrm{id} \otimes \nu_{q}\right)(\widetilde{\mathscr{R}})_{13},
\end{aligned}
$$

where in the last step we used $\left(\nu_{q} \otimes \nu_{q}\right)(\mathscr{R})=\mathscr{R}$, as the form $(-,-)$ is $\nu_{q}$-invariant.

It follows in particular from the first identity above that $\widetilde{\mathscr{E}}$ commutes with the $L_{\omega}^{+} L_{-\omega}^{-} \otimes 1$, hence

$$
\widetilde{\mathscr{E}} \in U_{q}^{10}\left(\mathfrak{u}_{\mathrm{r}} \oplus \mathfrak{a}\right) \hat{\otimes} U_{q}(\mathfrak{u}) .
$$

We write $\mathscr{E}$ for the projection of $\widetilde{\mathscr{E}}$ inside $U_{q}^{10}\left(\mathfrak{u}_{\mathrm{r}}\right) \hat{\otimes} U_{q}(\mathfrak{u})$. The following theorem is now immediate.

Theorem 3.24. The $\operatorname{Rep}_{q}(\mathfrak{u})$-module $C^{*}$-category $\operatorname{Rep}_{q}^{\nu}\left(\mathfrak{u}^{\nu}\right)$ is ribbon $\nu_{q}$-braided with respect to the $\nu_{q^{-}}$ braiding

$$
\eta_{M, U}(\xi \otimes \eta)=\mathscr{E}(\xi \otimes \eta), \quad \xi \in M, \eta \in U,
$$

where $M \in \operatorname{Rep}_{q}^{\nu}\left(\mathfrak{u}^{\nu}\right)$ and $U \in \operatorname{Rep}_{q}(\mathfrak{u})$. 


\section{Statement of the main conjecture}

We conjecture that the three ribbon twist-braided $\operatorname{Rep}_{q}(\mathfrak{u})$-module $\mathrm{C}^{*}$-categories constructed in the previous section are equivalent if the associated involutions of $\mathfrak{u}$ are inner equivalent.

Conjecture 4.1. Let $\mathfrak{u}$ be a compact semisimple Lie algebra and $\left\{e_{r}, f_{r}, h_{r} \mid r \in I\right\}$ be *-compatible Chevalley generators of $\mathfrak{g}=\mathfrak{u}^{\mathbb{C}}$. Let

- $\sigma$ be an involution of $\mathfrak{u}$ and $\mathfrak{k}_{\sigma}=\mathfrak{u}^{\sigma}$,

- $(X, \tau)$ be a Satake diagram with associated involution $\theta=\theta(X, \tau)$ and $\mathfrak{k}_{\theta}=\mathfrak{u}^{\theta}$.

- $(Y, \mu)$ be a Vogan diagram with associated involution $\nu=\nu(Y, \mu)$ and $\mathfrak{k}_{\nu}=\mathfrak{u}^{\nu}$.

Let $0<q<1$, and let $\hbar \in i \mathbb{R}$ be such that $e^{\pi i \hbar}=q$.

Then if $\sigma, \theta$ and $\nu$ are inner equivalent, we have

$$
\operatorname{Rep}_{\hbar}\left(\mathfrak{k}_{\sigma}\right) \cong \operatorname{Rep}_{q}^{\theta}\left(\mathfrak{k}_{\theta}\right) \cong \operatorname{Rep}_{q}^{\nu}\left(\mathfrak{k}_{\nu}\right)
$$

as twist-braided $\operatorname{Rep}_{q}(\mathfrak{u})$-module $C^{*}$-categories.

\section{THE RANK ONE CASE}

In this section we will verify Conjecture 4.1 for the simplest case $\mathfrak{u}=\mathfrak{s u}_{2}$. We first introduce some general terminology.

Definition 5.1. Let $\mathcal{C}$ be a tensor $\mathrm{C}^{*}$-category, and $\mathcal{D}$ a $\mathcal{C}$-module $\mathrm{C}^{*}$-category. We call $\mathcal{D}$ connected if for any nonzero objects $X, Y$ in $\mathcal{D}$ there exists $U$ in $\mathcal{C}$ with $\operatorname{Mor}(X \odot U, Y) \neq\{0\}$. For $X \in \mathcal{D}$ a simple object, we write $\mathcal{D}_{X}$ for the smallest $\mathrm{C}^{*}$-subcategory of $\mathcal{D}$ which is full, isomorphism-closed, and closed under taking subobjects, finite direct sums, and $\odot$.

Later, when we consider $\mathcal{D}=\operatorname{Rep}_{q}^{\mathbf{t}}(\mathfrak{k})$, we will write $\operatorname{Rep}_{q}^{\mathbf{t}}(\mathfrak{k} ; X)$ for $\mathcal{D}_{X}$.

The category $\mathcal{D}_{X}$ is itself a $\mathcal{C}$-module $\mathrm{C}^{*}$-category, and will be called the $\mathcal{C}$-module $\mathrm{C}^{*}$-category generated by $X$. If $\mathcal{C}$ is rigid, with $\bar{U}$ a dual object of $U$, it is clear by Frobenius reciprocity

$$
\operatorname{Mor}(X \odot U, Y) \cong \operatorname{Mor}(X, Y \odot \bar{U})
$$

that each $\mathcal{D}_{X}$ is equal to the connected full subcategory consisting of all objects $Y$ which appear as a subobject of $X \odot U$ for some $U$. Moreover, we then have $\mathcal{D}_{X}=\mathcal{D}_{Y}$ for each simple object $Y$ in $\mathcal{D}_{X}$. If $\mathcal{D}$ is twist-braided, this structure passes by restriction to any $\mathcal{D}_{X}$.

The following lemma is immediate.

Lemma 5.2. Let $\mathcal{C}$ be a rigid (braided) tensor $C^{*}$-category, and let $\mathcal{D}, \mathcal{D}^{\prime}$ be two (twist-braided) $\mathcal{C}$ module $C^{*}$-categories. Assume that $\left\{X_{i} \mid i \in \mathcal{I}\right\}$ and $\left\{Y_{i} \mid i \in \mathcal{I}\right\}$ are maximal collections of mutually non-isomorphic simple objects in respectively $\mathcal{D}$ and $\mathcal{D}^{\prime}$. If $\mathcal{D}_{X_{i}} \cong \mathcal{D}_{Y_{i}}^{\prime}$ for each $i \in \mathcal{I}$, then $\mathcal{D} \cong \mathcal{D}^{\prime}$.

Let us now turn to the specific case of $\mathfrak{u}=\mathfrak{s u}_{2}$. We use the standard generators $e, f, h$ for $\mathfrak{s l}_{2}(\mathbb{C})$,

$$
[h, e]=2 e, \quad[h, f]=-2 f, \quad[e, f]=h, \quad e^{*}=f, \quad h^{*}=h .
$$

In this case, there is up to inner equivalence only one non-trivial involution of $\mathfrak{s u}_{2}$, with Satake form and Vogan form determined by

$$
\theta(h)=-h, \quad \theta(e)=-f, \quad \theta(f)=-e, \quad \nu(h)=h, \quad \nu(e)=-e, \quad \nu(f)=-f .
$$

The respective fixed point subalgebras are

$$
\mathfrak{k}_{\theta}=\mathfrak{t}_{\theta}=\mathbb{R}(f-e), \quad \mathfrak{k}_{\nu}=\mathfrak{t}_{\nu}=i \mathbb{R} h,
$$

with complexifications $\mathfrak{h}_{\theta}=\mathbb{C}(f-e)$ and $\mathfrak{h}_{\nu}=\mathfrak{h}=\mathbb{C} h$.

Let us fix $0<q<1$ and $\hbar \in i \mathbb{R}$ with $q=e^{\pi i \hbar}$. Then $U_{q}\left(\mathfrak{s u}_{2}\right)$ is the Hopf $*$-algebra generated by $K, E, F$ with

$$
\begin{gathered}
K E=q^{2} E K, \quad K F=q^{-2} F K, \quad[E, F]=\frac{K-K^{-1}}{q-q^{-1}}, \quad K^{*}=K, \quad E^{*}=F K, \\
\Delta(K)=K \otimes K, \quad \Delta(E)=E \otimes 1+K \otimes E, \quad \Delta(F)=F \otimes K^{-1}+1 \otimes F .
\end{gathered}
$$

(Strictly speaking, we should also add a square root of $K$ by the conventions for $U_{q}(\mathfrak{u})$ used in this paper, but this will be inessential in what follows.) 
Let $V$ be the fundamental generating 2-dimensional *-representation of $U_{q}\left(\mathfrak{s u}_{2}\right)$ on $\mathbb{C}^{2}$ with the standard orthonormal basis $e_{+}=(1,0), e_{-}=(0,1)$ such that, with $X_{V}=\pi_{V}(X)$,

$$
E_{V}=\left(\begin{array}{cc}
0 & q^{1 / 2} \\
0 & 0
\end{array}\right), \quad F_{V}=\left(\begin{array}{cc}
0 & 0 \\
q^{-1 / 2} & 0
\end{array}\right), \quad K_{V}=\left(\begin{array}{cc}
q & 0 \\
0 & q^{-1}
\end{array}\right) .
$$

Then writing $e_{a b}=e_{a} \otimes e_{b}$, we have with respect to the basis $\left\{e_{++}, e_{+-}, e_{-+}, e_{--}\right\}$that the $R$-matrix and braiding at $V \otimes V$ are given by

$$
R=\mathscr{R}_{V, V}=q^{1 / 2}\left(\begin{array}{cccc}
q^{-1} & 0 & 0 & 0 \\
0 & 1 & q^{-1}-q & 0 \\
0 & 0 & 1 & 0 \\
0 & 0 & 0 & q^{-1}
\end{array}\right), \quad \beta=\beta_{V, V}=\Sigma R=q^{1 / 2}\left(\begin{array}{cccc}
q^{-1} & 0 & 0 & 0 \\
0 & 0 & 1 & 0 \\
0 & 1 & q^{-1}-q & 0 \\
0 & 0 & 0 & q^{-1}
\end{array}\right)
$$

Let us also introduce the following terminology.

Definition 5.3. Let $\mathcal{D}$ be a $\operatorname{Rep}_{q}\left(\mathfrak{s u}_{2}\right)$-module $\mathrm{C}^{*}$-category. We say that $\mathcal{D}$ has $\mathbb{Z}$-fusion rules if the simple objects of $\mathcal{D}$ (up to isomorphism) can be labeled $\left\{W_{n} \mid n \in \mathbb{Z}\right\}$ such that

$$
W_{n} \odot V \cong W_{n+1} \oplus W_{n-1} .
$$

Our first goal will now be to study the braided module $\mathrm{C}^{*}$-category structure on $\operatorname{Rep}_{q}^{\theta}\left(\mathfrak{k}_{\theta}\right)$. Note that in this case we have

$$
\mathcal{C}_{q, c}=\left\{q^{-2}\right\}, \quad \mathcal{S}_{q, c}=i \mathbb{R} .
$$

In the following, we identify $\mathcal{T}_{q, c}=\mathcal{C}_{q, c} \times \mathcal{S}_{q, c}$ with $\mathbb{R}$ by

$$
\left(q^{-2}, i t\right) \leftrightarrow t
$$

The coideal $U_{q}^{t}\left(\mathfrak{k}_{\theta}\right)$ is generated by

$$
B_{t}=F-q^{-2} E K^{-1}+i t K^{-1}, \text { and then } B_{t, V}=\left(\begin{array}{cc}
i t q^{-1} & -q^{-1 / 2} \\
q^{-1 / 2} & i t q
\end{array}\right) .
$$

Note that $B_{t}^{*}=-B_{t}$. In particular, the simple objects in $\operatorname{Rep}_{q}^{\theta}\left(\mathfrak{k}_{\theta}\right)$ are the evaluations

$$
\mathrm{ev}_{i t}: U_{q}^{0}\left(\mathfrak{k}_{\theta}\right) \rightarrow \mathbb{C}, \quad B_{0} \mapsto i t
$$

for $t \in \mathbb{R}$, and we then obtain equivalences of module $\mathrm{C}^{*}$-categories with fixed generating object

$$
\operatorname{Rep}_{q}^{\theta}\left(\mathfrak{k}_{\theta} ; \operatorname{ev}_{i t}\right) \cong \operatorname{Rep}_{q}^{t}\left(\mathfrak{k}_{\theta} ; \varepsilon\right),
$$

where $\varepsilon$ is the counit of $U_{q}\left(\mathfrak{s u}_{2}\right)$ restricted to $U_{q}^{t}\left(\mathfrak{k}_{\theta}\right)$.

For $t \in \mathbb{R}$, let us write $\lambda=\lambda_{t} \in \mathbb{R}$ for the unique real number such that

$$
t=q^{-1 / 2} \frac{q^{-\lambda}-q^{\lambda}}{q^{-1}-q} .
$$

Lemma 5.4. Let $\mathbb{C}_{n}$ be the one-dimensional $*$-representation of $U_{q}^{t}\left(\mathfrak{k}_{\theta}\right)$ given by the $*$-character

$$
\chi_{n}: U_{q}^{t}\left(\mathfrak{k}_{\theta}\right) \rightarrow \mathbb{C}, \quad B_{t} \mapsto i q^{-1 / 2} \frac{q^{-\lambda-n}-q^{\lambda+n}}{q^{-1}-q} .
$$

Then the $\mathbb{C}_{n}$ exhaust up to isomorphism the irreducible objects in $\operatorname{Rep}_{q}^{t}\left(\mathfrak{k}_{\theta} ; \varepsilon\right)$, and

$$
\mathbb{C}_{n} \odot V \cong \mathbb{C}_{n+1} \oplus \mathbb{C}_{n-1},
$$

that is, $\operatorname{Rep}_{q}^{t}\left(\mathfrak{k}_{\theta} ; \varepsilon\right)$ has $\mathbb{Z}$-fusion rules.

Proof. Clearly $\mathbb{C}_{0}$ corresponds to the trivial representation given by the counit. Then the fusion rules above follow from an easy spectral computation for the 2-by-2-matrices $\left(\chi_{n} \otimes \pi_{V}\right) \Delta\left(B_{t}\right)$. Since $\mathbb{C}_{0}$ is generating, these exhaust all irreducible objects.

Proposition 5.5. Let $t \in \mathbb{R}$. Up to isomorphism, there is a unique ribbon braided $\operatorname{Rep}_{q}\left(\mathfrak{s u}_{2}\right)$-module $C^{*}$-category structure $\eta$ on $\operatorname{Rep}_{q}^{t}\left(\mathfrak{k}_{\theta} ; \varepsilon\right)$ such that $C=\eta_{\varepsilon, V}$ is not a scalar multiple of the identity. In that case, the singular values of $C$ are $\left\{q^{ \pm \lambda-1 / 2}\right\}$. 
Proof. From the defining relations (1.1) and (1.2) (in the case of trivial braided monoidal autoequivalence), it is clear that $\eta$ is completely determined by $C=\eta_{\varepsilon, V}$. Moreover, as $\eta$ is a natural transformation,

$$
\eta_{\varepsilon, V \otimes V}=(C \otimes 1) \beta(C \otimes 1) \beta=\beta(C \otimes 1) \beta(C \otimes 1) .
$$

It is immediate then from a direct computation, cf. tDHO98, Section 5], that, if $C$ is not diagonal, $C$ must be of the form 10

$$
C=\left(\begin{array}{ll}
a & b \\
c & 0
\end{array}\right)
$$

with $b c=-q^{-1}$. But as $C$ must commute with $B_{t, V}$, either $C$ is a scalar multiple of the identity (which is excluded by assumption) or

$$
C= \pm\left(\begin{array}{cc}
i t\left(q^{-1}-q\right) & -q^{-1 / 2} \\
q^{-1 / 2} & 0
\end{array}\right)
$$

Hence the singular values of $C$ are indeed $\left\{q^{ \pm \lambda-1 / 2}\right\}$. The sign of $C$ is irrelevant as it can be changed by means of the natural automorphism $F$ of the identity functor on $\operatorname{Rep}_{q}\left(\mathfrak{s u}_{2}\right)$ characterized by

$$
F_{U_{n / 2}}=(-1)^{n} \operatorname{id}_{U_{n / 2}}
$$

where $U_{n / 2}$ is the unique (up to isomorphism) irreducible representation of dimension $n+1$.

We want to generalize the above computation to ribbon (twist-)braided module categories with $\mathbb{Z}$ fusion rules. Assume that a (twist-)braided $\operatorname{Rep}_{q}\left(\mathfrak{s u}_{2}\right)$-module category $(\mathcal{D}, \odot, \Psi, \eta)$ has $\mathbb{Z}$-fusion rules, with simple objects $\left\{W_{n} \mid n \in \mathbb{Z}\right\}$. With $C=\eta_{W_{0}, V}$, the morphism $C^{*} C$ is a positive invertible element in the $\mathrm{C}^{*}$-algebra $\mathcal{D}\left(W_{0} \odot V\right) \cong \mathbb{C}^{2}$, which must be represented by $(a, b)$ with $a, b>0$. We then write $\operatorname{Tr}\left(C^{*} C\right)=a+b$, which agrees with the usual matricial trace in the case of $\mathcal{D}=\operatorname{Rep}_{q}^{t}\left(\mathfrak{k}_{\theta} ; \varepsilon\right)$.

Theorem 5.6. Let $(\mathcal{D}, \odot, \Psi, \eta)$ be a ribbon braided $\left(\operatorname{Rep}_{q}\left(\mathfrak{s u}_{2}\right), \otimes, \beta\right)$-module $C^{*}$-category with $\mathbb{Z}$-fusion rules. Under the above notation, assume that $C=\eta_{W_{0}, V}$ is non-scalar, and let $t \in \mathbb{R}$ be a number satisfying

$$
\operatorname{Tr}\left(C^{*} C\right)=q^{-1}\left(q^{2 \lambda_{t}}+q^{-2 \lambda_{t}}\right) .
$$

Then $\mathcal{D}$ and $\operatorname{Rep}_{q}^{t}\left(\mathfrak{k}_{\theta} ; \varepsilon\right)$ are equivalent as braided $\operatorname{Rep}_{q}\left(\mathfrak{s u}_{2}\right)$-module categories by an equivalence sending $W_{0}$ to $\varepsilon=\mathbb{C}_{0}$.

Note that there are two possibilities for $t$. These two choices are related by the equivariant $*-$ isomorphism $U_{q}^{t}\left(\mathfrak{k}_{\theta}\right) \rightarrow U_{q}^{-t}\left(\mathfrak{k}_{\theta}\right)$ extending $B_{t} \mapsto B_{-t}$, which corresponds to an equivalence of module categories $\operatorname{Rep}_{q}^{t}\left(\mathfrak{k}_{\theta} ; \varepsilon\right) \rightarrow \operatorname{Rep}_{q}^{-t}\left(\mathfrak{k}_{\theta} ; \varepsilon\right)$ sending $\mathbb{C}_{n}$ to $\mathbb{C}_{-n}$ (see also the proof below).

Proof. Let us first recall a combinatorial classification of the $\operatorname{Rep}_{q}\left(\mathfrak{s u}_{2}\right)$-module $\mathrm{C}^{*}$-categories with $\mathbb{Z}$ fusion rules. Let $R_{V}$ be the (unique up to scalar) morphism $\mathbb{1} \rightarrow V \otimes V$ in $\operatorname{Rep}_{q}\left(\mathfrak{s u}_{2}\right)$ satisfying $R_{V}^{*} R_{V}=q+q^{-1}$. Concretely, we may represent $R_{V}$ by the map

$$
\mathbb{C} \rightarrow V \otimes V, \quad \alpha \mapsto \alpha\left(q^{-1 / 2} e_{+} \otimes e_{-}-q^{1 / 2} e_{-} \otimes e_{+}\right)
$$

so that $\beta_{V, V}=q^{1 / 2}\left(q^{-1}-R_{V} R_{V}^{*}\right)$.

Let $\mathcal{D}$ be a module category with $\mathbb{Z}$-fusion rules, with simple objects $\left\{W_{n} \mid n \in \mathbb{Z}\right\}$. Then, when $n=m \pm 1$, we have a conjugate linear map

$$
\mathcal{J}_{m, n}: \mathcal{D}\left(W_{m}, W_{n} \odot V\right) \rightarrow \mathcal{D}\left(W_{n}, W_{m} \odot V\right), \quad T \mapsto\left(T^{*} \odot V\right) \Psi^{-1}\left(W_{n} \odot R_{V}\right) .
$$

Then $w_{m, n}=\mathcal{J}_{m, n}^{*} \mathcal{J}_{m, n}$ is a positive scalar subject to conditions $w_{m, n} w_{n, m}=1$ (for $n=m \pm 1$ ) and $w_{m, m+1}+w_{m, m-1}=q+q^{-1}$ for all $m$. These relations determine the weights $w_{m, n}$ from $w_{0,1}$ alone, and the open interval $\left(q, q^{-1}\right)$ is the possible range of $w_{0,1}$ (recall that we assumed $0<q<1$ ). Moreover, $w_{0,1}$ is a complete invariant of module categories with $\mathbb{Z}$-fusion rules, in the sense that if $\mathcal{D}^{\prime}$ is another $\operatorname{Rep}_{q}\left(\mathfrak{s u}_{2}\right)$-module category with $\mathbb{Z}$-fusion rules, with simple objects $\left\{W_{n}^{\prime} \mid n \in \mathbb{Z}\right\}$ and the associated sequence of positive numbers $\left\{w_{m, n}^{\prime} \mid n=m \pm 1\right\}$ with $w_{0,1}^{\prime}=w_{0,1}$, there is an essentially unique equivalence of module categories $F: \mathcal{D} \rightarrow \mathcal{D}^{\prime}$ such that $F\left(W_{n}\right)=W_{n}^{\prime}$ for all $n$, see $[\mathrm{DCY} 15$ ] (in particular Section 2 and Example 3.12) for more details.

To connect the number $w_{0,1}$ to ribbon twist braids, let us consider the (normalized) partial categorical traces

$$
W_{0} \otimes \operatorname{tr}: \mathcal{D}\left(W_{0} \odot V\right) \rightarrow \mathcal{D}\left(W_{0}\right), \quad T \mapsto \frac{1}{q+q^{-1}}\left(W_{0} \odot R_{V}^{*}\right) \Psi(T \odot V) \Psi^{-1}\left(W_{0} \odot R_{V}\right)
$$

\footnotetext{
${ }^{10}$ The specific form differs from that in $\mathrm{tDHO} 98$ as a different convention for the $R$-matrix is used.
} 
Since $\mathcal{D}\left(W_{0}\right) \cong \mathbb{C}$, this can be regarded as a faithful state on $\mathcal{D}\left(W_{0} \odot V\right) \cong \mathbb{C}^{2}$, which should be of the form $(a, b) \mapsto s a+(1-s) b$ for a unique $0<s<1$. Unpacking the definitions, we see that the numbers $w_{0, \pm 1}$ are equal to $s\left(q+q^{-1}\right)$ and $(1-s)\left(q+q^{-1}\right)$.

Now, in the module category $\operatorname{Rep}_{q}^{t}\left(\mathfrak{t}_{\theta} ; \varepsilon\right)$ we have a morphism $\eta_{\varepsilon, V}$ represented by the matrix (5.1). Projections onto its eigenspaces gives the desired direct product decomposition of $\operatorname{End}_{\operatorname{Rep}_{q}^{t}\left(\mathfrak{t}_{\theta}\right)}\left(\mathbb{C}_{0} \otimes V\right)$ as an algebra. Combined with the above concrete formula for $R_{V}$, we see that the associated weights satisfy

$$
\left\{w_{0,1}, w_{0,-1}\right\}=\left\{\frac{q^{\lambda_{t}+1}+q^{-\left(\lambda_{t}+1\right)}}{q^{\lambda_{t}}+q^{-\lambda_{t}}}, \frac{q^{\lambda_{t}-1}+q^{-\left(\lambda_{t}-1\right)}}{q^{\lambda_{t}}+q^{-\lambda_{t}}}\right\} .
$$

Since $\left(q^{\lambda+1}+q^{-(\lambda+1)}\right) /\left(q^{\lambda}+q^{-\lambda}\right)$ is a monotone increasing function in $\lambda$ whose range is between $q$ and $q^{-1}$, we see that any possible value of weight $w_{0,1}$ is realized by some module category $\operatorname{Rep}_{q}^{t}\left(\mathfrak{t}_{\theta} ; \varepsilon\right)$ for a unique $t$ if we take $W_{n}=\mathbb{C}_{n}$. Thus, the module categories $\operatorname{Rep}_{q}^{t}\left(\mathfrak{k}_{\theta} ; \varepsilon\right)$ for different $t$ exhaust all possible $\operatorname{Rep}_{q}\left(\mathfrak{s u}_{2}\right)$-module categories with $\mathbb{Z}$-fusion rules.

Suppose $(\mathcal{D}, \odot, \Psi, \eta)$ is given as in the assertion, and let $t$ be such that there is an equivalence of module categories $F: \mathcal{D} \rightarrow \operatorname{Rep}_{q}^{t}\left(\mathfrak{k}_{\theta} ; \varepsilon\right)$ such that $F\left(W_{0}\right)=\mathbb{C}_{0}$. Then $F\left(\eta_{W_{0}, V}\right)$ should be of the form (5.1) as $t$ is determined by $w_{0,1}$. Since $\eta_{\varepsilon, V}$ determines $\eta$, we obtain the assertion.

The following theorem now settles one part of Conjecture 4.1 in this case.

Theorem 5.7. Let $\sigma$ be an involution of $\mathfrak{s u}_{2}$. Then $\operatorname{Rep}_{\hbar}\left(\mathfrak{k}_{\sigma}\right)$ and $\operatorname{Rep}_{q}^{\theta}\left(\mathfrak{k}_{\theta}\right)$ are equivalent as twist-braided module $C^{*}$-categories.

Proof. By Remark 3.9 and the fact that all involutions of $\mathfrak{s u}_{2}$ are inner, we may take $\sigma=\theta=\operatorname{Ad}(g)$ with

$$
g=\left(\begin{array}{cc}
0 & 1 \\
-1 & 0
\end{array}\right)
$$

Clearly, we can take as a maximal family of simple objects in $\operatorname{Rep}_{\hbar}\left(\mathfrak{k}_{\theta}\right)$ the family of characters

$$
\chi_{\lambda}: \mathfrak{h}_{\theta} \rightarrow \mathbb{C}, \quad f-e \mapsto i \lambda,
$$

for $\lambda \in \mathbb{R}$. By Lemma 5.2 , it is now sufficient to show that

$$
\operatorname{Rep}_{\hbar}\left(\mathfrak{k}_{\theta} ; \chi_{\lambda}\right) \cong \operatorname{Rep}_{q}^{\theta}\left(\mathfrak{k}_{\theta} ; \operatorname{ev}_{i t}\right) \text { for } \lambda=\lambda_{t} .
$$

By Remark 1.13. we know that $\operatorname{Rep}_{\hbar}\left(\mathfrak{k}_{\theta} ; \chi_{\lambda}\right)$ is a ribbon (Id-)braided module category over $\operatorname{Rep}_{\hbar}\left(\mathfrak{s u}_{2}\right)$, with associated non-scalar braiding

$$
\begin{aligned}
\widetilde{\eta}_{\chi_{\lambda}, V}=e^{i \pi \hbar\left(\chi_{\lambda}(f-e) \pi_{V}(f-e)-\frac{1}{2}\right)} g=e^{-\pi i \hbar / 2}\left(\begin{array}{cc}
\cos (\pi \hbar \lambda) & \sin (\pi \hbar \lambda) \\
-\sin (\pi \hbar \lambda) & \cos (\pi \hbar \lambda)
\end{array}\right) g & \\
& =\frac{q^{-1 / 2}}{2}\left(\begin{array}{cc}
i\left(q^{\lambda}-q^{-\lambda}\right) & q^{\lambda}+q^{-\lambda} \\
-\left(q^{\lambda}+q^{-\lambda}\right) & i\left(q^{\lambda}-q^{-\lambda}\right)
\end{array}\right) .
\end{aligned}
$$

Since $\operatorname{Rep}_{\hbar}\left(\mathfrak{k}_{\theta} ; \chi_{\lambda}\right)$ has $\mathbb{Z}$-fusion rules with $\operatorname{Tr}\left(\widetilde{\eta}_{\chi_{\lambda}, V}^{*} \widetilde{\eta}_{\chi_{\lambda}, V}\right)=q^{-1}\left(q^{2 \lambda}+q^{-2 \lambda}\right)$, we conclude that

$$
\operatorname{Rep}_{\hbar}\left(\mathfrak{k}_{\theta} ; \chi_{\lambda}\right) \cong \operatorname{Rep}_{q}^{t}\left(\mathfrak{k}_{\theta} ; \varepsilon\right) \cong \operatorname{Rep}_{q}^{\theta}\left(\mathfrak{k}_{\theta} ; \operatorname{ev}_{i t}\right)
$$

as twist-braided $\operatorname{Rep}_{q}\left(\mathfrak{s u}_{2}\right)$-module $\mathrm{C}^{*}$-categories by Proposition 5.5 and Theorem 5.6

Let us now prove the second part of Conjecture 4.1 . Note first that in this case, $U_{q}^{10}\left((\mathfrak{s u})_{\mathrm{r}}\right)$ is given by the universal relations

$$
K F=q^{-2} F K, \quad K F^{*}=q^{2} F^{*} K, \quad F^{*} F-q^{2} F F^{*}=\frac{1+K^{-2}}{q-q^{-1}} .
$$

The following lemma is straightforward.

Lemma 5.8. The weights

$$
\omega_{r}(K)=q^{-r}, \quad r \in \mathbb{R}
$$

exhaust the family of admissible weights for $U_{q}^{10}\left(\left(\mathfrak{s u}_{2}\right)_{\mathrm{r}}\right)$. 
Proof. Let $M_{r}=\mathbb{C}[\mathbb{N}]$ be a pre-Hilbert space (with $\left\{e_{n}\right\}_{n \in \mathbb{N}}$ being an orthonormal basis) endowed with the *-representation $\pi_{r}$ of $U_{q}^{10}\left(\left(\mathfrak{s u}_{2}\right)_{\mathrm{r}}\right)$ characterized by

$$
\pi_{r}(K) e_{n}=q^{-r+2 n} e_{n}, \quad q^{1 / 2}\left(q^{-1}-q\right) \pi_{r}(F) e_{n}=q^{-n}\left(\left(1-q^{2 n}\right)\left(1+q^{2 r+2-2 n}\right)\right)^{1 / 2} e_{n-1} .
$$

It can be concretely verified that these exhaust the isomorphism classes of irreducible $*$-representations of $U_{q}^{10}\left(\left(\mathfrak{s u}_{2}\right)_{\mathrm{r}}\right)$.

We will keep the notation $\left(M_{r}, \pi_{r}\right)$ from the proof of the previous lemma.

Lemma 5.9. We have $M_{r} \odot V \cong M_{r+1} \oplus M_{r-1}$.

Proof. Let $\eta_{r}$ be the lowest weight vector in $M_{r}$. Then the $U_{q}^{10}\left(\left(\mathfrak{s u}_{2}\right)_{\mathrm{r}}\right)$-module $M_{r} \otimes V$ is generated by the lowest weight vector $\eta_{r} \otimes e_{-}$(of weight $\omega_{r+1}$ ) and $\eta_{r} \otimes e_{+}$. But the image of $\eta_{r} \otimes e_{+}$in the quotient module $\left(M_{r} \otimes V\right) / U_{q}^{10}\left(\left(\mathfrak{s u}_{2}\right)_{\mathrm{r}}\right)\left(\eta_{r} \otimes e_{-}\right)$is a lowest weight vector of weight $\omega_{r-1}$.

The following theorem now settles the second part of Conjecture 4.1 .

Theorem 5.10. The ribbon twist-braided $\operatorname{Rep}_{q}\left(\mathfrak{s u}_{2}\right)$-module $C^{*}$-categories $\operatorname{Rep}_{q}^{\theta}\left(\mathfrak{k}_{\theta}\right)$ and $\operatorname{Rep}_{q}^{\nu}\left(\mathfrak{k}_{\nu}\right)$ are equivalent.

Proof. By Lemma 5.9 and Lemma 5.2 , it suffices to show that the $\nu_{q}$-braided $\operatorname{Rep}_{q}\left(\mathfrak{s u}_{2}\right)$-module $\mathrm{C}^{*}$ category $\operatorname{Rep}_{q}^{\nu}\left(\mathfrak{k}_{\nu} ; M_{r}\right)$ is equivalent to $\operatorname{Rep}_{q}^{\theta}\left(\mathfrak{k}_{\theta} ; \operatorname{ev}_{i t}\right) \cong \operatorname{Rep}_{q}^{t}\left(\mathfrak{k}_{\theta} ; \varepsilon\right)$ for $\lambda_{t}=r+1$.

Now the ribbon element $v$ for $U_{q}\left(\mathfrak{s u}_{2}\right)$ satisfies

$$
v_{V}=q^{3 / 2} .
$$

On the other hand, we can formally write $K=q^{H}$ and

$\left(\mathrm{id} \otimes \nu_{q}\right)(\widetilde{\mathscr{R}})=\left(1 \otimes 1+\left(q-q^{-1}\right) K F^{*} \otimes F+\ldots\right) q^{-\frac{1}{2} H \otimes H}, \quad \widetilde{\mathscr{R}}_{21}=\left(1 \otimes 1-\left(q-q^{-1}\right) F \otimes E+\ldots\right) q^{-\frac{1}{2} H \otimes H}$ interpreted in $U_{q}^{10}\left(\left(\mathfrak{s u}_{2}\right)_{\mathrm{r}}\right) \otimes U_{q}\left(\mathfrak{s u}_{2}\right)$ (as the extra Cartan part of $U_{q}^{10}(\mathfrak{u} \oplus \mathfrak{a})$ is central in this case, it can be ignored in the discussion), hence

$$
\left(\mathrm{id} \otimes \nu_{q}\right)(\widetilde{\mathscr{R}})\left(\eta_{r} \otimes e_{-}\right)=q^{-\frac{r}{2}} \eta_{r} \otimes e_{-}, \quad\left(\mathrm{id} \otimes \nu_{q}\right)(\widetilde{\mathscr{R}})\left(\eta_{r} \otimes e_{+}\right)=q^{\frac{r}{2}}\left(\eta_{r} \otimes e_{+}+\left(q-q^{-1}\right) q^{\frac{3}{2}-r} F^{*} \eta_{r} \otimes e_{-}\right) .
$$

It follows that with the $\nu_{q}$-braiding $\mathscr{E}=\mathscr{R}_{21}\left(\mathrm{id} \otimes \nu_{q}\right) \mathscr{R}\left(1 \otimes v^{-1}\right)$, we have

$$
\begin{aligned}
& \mathscr{E}\left(\eta_{r} \otimes e_{-}\right)=q^{-r-\frac{3}{2}} \eta_{r} \otimes e_{-}, \\
& \mathscr{E}\left(\eta_{r} \otimes e_{+}\right)=q^{\frac{r-3}{2}}\left(\left(\left(q-q^{-1}\right) q^{1-\frac{3}{2} r}+q^{2+\frac{1}{2} r}\right) \eta_{r} \otimes e_{+}+\left(q-q^{-1}\right) q^{\frac{5-3 r}{2}} F^{*} \eta_{r} \otimes e_{-}\right) .
\end{aligned}
$$

Since in the quotient module $\left(M_{r} \otimes V\right) / U_{q}^{10}\left((\mathfrak{s u})_{\mathrm{r}}\right)\left(\eta_{r} \otimes e_{-}\right)$we can identify $F^{*} \eta_{r} \otimes e_{-}$and $-q^{-3 / 2} \eta_{r} \otimes$ $e_{+}$, it follows that $\mathscr{E}$ has eigenvalues $q^{-r-\frac{3}{2}}$ and $q^{r+\frac{1}{2}}$, which are also its singular values. Now, we can write

$$
\nu_{q}=\operatorname{Ad}\left(K_{\chi}\right), \text { where } \chi(H)=\frac{\pi i}{2 \ln (q)} .
$$

As the element $\mathscr{E} K_{\chi}$ is not scalar and has the same singular values as $\mathscr{E}$, in view of Remark 1.13 . Theorem 5.6 implies that $\operatorname{Rep}_{q}^{\nu}\left(\mathfrak{s u}_{2} ; M_{r}\right)$ is equivalent to $\operatorname{Rep}_{q}^{t}\left(\mathfrak{k}_{\theta} ; \varepsilon\right)$ for $t$ such that $\lambda_{t}=r+1$, by an equivalence sending $M_{r}$ to $\mathbb{C}_{0}$.

\section{Appendix A. Symmetric PAIRS}

A.1. Proof of Theorem 2.6. Let $\theta$ be any involution of $\mathfrak{u}$. Since any automorphism of $\mathfrak{u}$ is the composition of an inner automorphism and the automorphism induced from a Dynkin diagram automorphism, for the existence part it is enough to show that one can find $*$-compatible Chevalley generators of $\mathfrak{g}$ with respect to which $\theta$ is in Satake form.

Denote the eigenspace decomposition of $\theta$ by $\mathfrak{u}=\mathfrak{k} \oplus \mathfrak{m}$, with $\mathfrak{k}=\mathfrak{u}^{\theta}$, and consider the real form

$$
\mathfrak{g}_{\theta}=\mathfrak{k} \oplus i \mathfrak{m}
$$

of $\mathfrak{g}$. It is a real semisimple Lie algebra and $\theta$ is a Cartan involution on it. Let $\mathfrak{t} \subset \mathfrak{u}$ be a $\theta$-invariant Cartan subalgebra such that, with $\mathfrak{h}=\mathfrak{t}^{\mathbb{C}}$, the Cartan subalgebra $\mathfrak{h} \cap \mathfrak{g}_{\theta}$ of $\mathfrak{g}_{\theta}$ is maximally split, that is, it contains a maximal abelian subspace of $i \mathfrak{m}$. Specifically, we start with a maximal abelian subspace $\mathfrak{a}_{\theta} \subseteq i \mathfrak{m}$, then take a maximal abelian subalgebra $\mathfrak{t}_{\theta}$ of the centralizer of $\mathfrak{a}_{\theta}$ in $\mathfrak{k}$, and then define $\mathfrak{t}=\mathfrak{t}_{\theta} \oplus i \mathfrak{a}_{\theta}$, see Kna02, Proposition 6.47].

The real vector space spanned by the root vectors can be identified with $\left(i \mathfrak{t}_{\theta}\right)^{*} \oplus \mathfrak{a}_{\theta}^{*}$. Consider the lexicographic order on this space defined by a basis $x_{1}, \ldots, x_{n}$ in $i \mathfrak{t}_{\theta} \oplus \mathfrak{a}_{\theta}$ such that $x_{1}, \ldots, x_{p}$ is a basis 
in $\mathfrak{a}_{\theta}$. Consider the corresponding positive roots $\alpha \in \Delta^{+}$and simple roots $\alpha_{r}, r \in I$. This already fixes the generators $h_{r}$. For a root $\alpha$, denote by $\bar{\alpha}$ the restriction of $\alpha$ to $\mathfrak{a}_{\theta}$. Let

$$
X=\left\{r \in I \mid \overline{\alpha_{r}}=0\right\} .
$$

Recall that $\Theta: \mathfrak{h}^{*} \rightarrow \mathfrak{h}^{*}$ defines the involutive transformation dual to $\theta_{\mid \mathfrak{h}}$. It acts trivially on the root system $\Delta_{X}$ generated by $X$. From the definition of the order structure it is also clear that $\Theta$ maps every root $\alpha \in \Delta^{+} \backslash \Delta_{X}^{+}$into a negative root. It follows that $-w_{X} \circ \Theta$ maps $\Delta^{+}$onto itself, so

$$
-w_{X} \circ \Theta=\tau
$$

for an automorphism $\tau$ of the Dynkin diagram such that its action on $X$ coincides with that of $-w_{X}$. In other words, if we fix generators $e_{r}$ and $f_{r}$ (such that $f_{r}=e_{r}^{*}$ ) and define the element $m_{X}$ using them, then the automorphism $\theta \circ\left(\operatorname{Ad} m_{X}\right)^{-1} \circ \tau^{-1} \circ \omega$ of $\mathfrak{g}$ is trivial on $\mathfrak{h}$. Hence

$$
\theta=(\operatorname{Ad} z) \circ \omega \circ \tau \circ\left(\operatorname{Ad} m_{X}\right)=(\operatorname{Ad} z) \circ\left(\operatorname{Ad} m_{X}\right) \circ \tau \circ \omega,
$$

for some $z \in T$, where we used that $\tau\left(m_{X}\right)=m_{X}$ by $\tau$-invariance of $X$ and that the automorphisms Ad $m_{X}$ and $\omega$ commute Kol14, Proposition 2.2(3)].

Next we want to show that $z_{k}=1$ for $k \in X$. Let us prove first that $\theta\left(e_{k}\right)=e_{k}$ for such $k$. Assume this is not the case for some $k \in X$. Since the root space $\mathfrak{g}_{\alpha_{k}}$ is one-dimensional and $\theta$-invariant, it follows that $\theta\left(e_{k}\right)=-e_{k}$. But then the element $e_{k}+f_{k}$ lies in $i \mathfrak{m}$ and commutes with $\mathfrak{a}_{\theta}$, which contradicts the maximality of $\mathfrak{a}_{\theta}$.

Denote by $\tau_{X}$ the permutation of $X$ defined by $-w_{X}$. Then on the Lie subalgebra $\mathfrak{g}_{X} \subset \mathfrak{g}$ corresponding to $X$ we have

$$
\operatorname{Ad} z=\theta \circ \omega \circ \tau^{-1} \circ\left(\operatorname{Ad} m_{X}\right)^{-1}=\omega \circ \tau_{X} \circ\left(\operatorname{Ad} m_{X}\right)^{-1}=\mathrm{id},
$$

where the last equality follows, e.g., from BV+95, Lemme 4.9]. This shows that $z_{k}=1$ for $k \in X$.

Finally, it remains to show that $\tau$ is involutive. Since $\theta$ is trivial on $\mathfrak{g}_{X}$, the automorphisms $\theta$ and $\operatorname{Ad} m_{X}$ commute. Hence the transformations $\Theta$ and $w_{X}$ commute. But then the equality $-w_{X} \circ \Theta=\tau$ implies that $\tau$ is involutive.

As already explained in Kol14, uniqueness of $(X, \tau)$ up to conjugation follows from results of Kac and Wang [KW92].

A.2. Proof of Theorem 2.8. Given an involution $\nu$ of $\mathfrak{u}$, it is sufficient to show that there exist Chevalley generators with respect to which $\nu$ is in Vogan form.

Again as above consider the eigenspace decomposition $\mathfrak{u}=\mathfrak{k} \oplus \mathfrak{m}$ of $\nu$ and the real form $\mathfrak{g}_{\nu}=\mathfrak{k} \oplus i \mathfrak{m}$ of $\mathfrak{g}$. Choose a $\nu$-invariant Cartan subalgebra $\mathfrak{t}$ of $\mathfrak{u}$ with complexification $\mathfrak{h}$ such that $\mathfrak{h} \cap \mathfrak{g}_{\nu}$ is maximally anisotropic in $\mathfrak{g}_{\nu}$, that is, it contains a Cartan subalgebra of $\mathfrak{k}$. Specifically, we start with a Cartan subalgebra $\mathfrak{t}_{\nu}$ of $\mathfrak{k}$, then take its centralizer in $\mathfrak{g}_{\nu}$, which necessarily has the form $\mathfrak{t}_{\nu} \oplus \mathfrak{a}_{\nu}$ for some $\mathfrak{a}_{\nu} \subseteq i \mathfrak{m}$, and put $\mathfrak{t}=\mathfrak{t}_{\nu} \oplus i \mathfrak{a}_{\nu}$, see Kna02, Proposition 6.60].

Every root $\alpha$ with respect to $\mathfrak{t}$ restricts to a nonzero functional on $\mathfrak{t}_{\nu}$. Indeed, assume that this is not the case for some $\alpha$. Then for the transformation $N$ dual to $\nu_{\mid \mathfrak{h}}$ we have $N(\alpha)=-\alpha$, hence $\nu\left(\mathfrak{g}_{\alpha}\right)=\mathfrak{g}_{-\alpha}$. Since $\nu$ is unitary with respect to the inner product $B\left(X, Y^{*}\right)$ on $\mathfrak{g}$, for any nonzero $e_{\alpha} \in \mathfrak{g}_{\alpha}$ we have $\nu\left(e_{\alpha}\right)=c_{\alpha} e_{\alpha}^{*}$ for some $c_{\alpha} \in \mathbb{T}$. By rescaling $e_{\alpha}$ we may assume that $c_{\alpha}=1$. But then $i\left(e_{\alpha}+e_{\alpha}^{*}\right)$ lies in $\mathfrak{k}$ and commutes with $\mathfrak{t}_{\nu}$, which contradicts the maximality of $\mathfrak{t}_{\nu}$.

Now, consider the lexicographic order on $(i \mathfrak{t})^{*}=\left(i \mathfrak{t}_{\nu}\right)^{*} \oplus \mathfrak{a}_{\nu}^{*}$ defined by a basis $x_{1}, \ldots, x_{n}$ in $i \mathfrak{t}_{\nu} \oplus \mathfrak{a}_{\nu}$ such that $x_{1}, \ldots, x_{l}$ is a basis in $i \mathfrak{t}_{\nu}$. Consider the corresponding system of simple roots. This fixes the generators $h_{r}$. By the previous paragraph, the set of positive roots is invariant under $N$, hence $N$ is defined by an involutive automorphism $\mu$ of the Dynkin diagram.

Choose Chevalley generators $e_{r}$ and $f_{r}$ such that $e_{r}^{*}=f_{r}$. If $\alpha_{r}$ is identically zero on $\mathfrak{a}_{\nu}$, then $N\left(\alpha_{r}\right)=\alpha_{r}$ and the root space $\mathfrak{g}_{\alpha_{r}}$ is $\nu$-invariant. Hence either $\nu\left(e_{r}\right)=e_{r}$ or $\nu\left(e_{r}\right)=-e_{r}$. On the other hand, if $\alpha_{r}$ restricts to a nonzero functional on $\mathfrak{a}_{\nu}$, then $\alpha_{\mu(r)}=N\left(\alpha_{r}\right) \neq \alpha_{r}$ and $\nu\left(e_{r}\right)=c_{r} e_{\mu(r)}$ for some $c_{r} \in \mathbb{T}$. But then by choosing a representative $r$ in every 2-point orbit of $\mu$ and by replacing $e_{\mu(r)}$ by $c_{r} e_{\mu(r)}$, and correspondingly $f_{\mu(r)}$ by $\bar{c}_{r} f_{\mu(r)}$, we can arrange that $\nu\left(e_{r}\right)=e_{\mu(r)}$.

\section{APPENDIX B. *-INVARIANCE OF QUANTUM COIDEAL SYMMETRIC PAIRS}

In this section we will prove Theorem 3.11

We will need some more information on the Lusztig braid operators. Following [Jan96, Section 8], let us also write $T_{r}$ for the braid operators as acting on finite admissible $U_{q}(\mathfrak{g})$-modules $V$, so

$$
T_{r}(x v)=T_{r}(x) T_{r}(v), \quad\left(x \in U_{q}(\mathfrak{g}), v \in V\right) .
$$


Let again

$$
w_{X}=s_{r_{1}} \cdots s_{r_{M}}
$$

be a reduced expression of the longest element of the Weyl group of $\mathfrak{g}_{X}$. Then we have $w_{X}=s_{r_{M}} \cdots s_{r_{1}}$,

$$
\beta_{1}=\alpha_{r_{1}}, \quad \beta_{2}=s_{r_{1}}\left(\alpha_{r_{2}}\right), \quad \beta_{3}=s_{r_{1}} s_{r_{2}}\left(\alpha_{r_{3}}\right), \quad \ldots, \quad \beta_{M}=s_{r_{1}} \cdots s_{r_{M-1}}\left(\alpha_{r_{M}}\right)
$$

is an enumeration of the positive roots of $\mathfrak{g}_{X}$ Bou07a, Section V.1.6, Corollaire 2 à Proposition 17], and $T_{w_{X}}=T_{r_{1}} \cdots T_{r_{M}}$ is independent of the choice of reduced expression Lus93, Chapter 39; Jan96, Section 8.18-8.23].

For $\varpi$ a dominant integral weight of $\mathfrak{g}_{X}$, let $V_{\varpi}$ be the highest weight $U_{q}\left(\mathfrak{g}_{X}\right)$-module with weight $\varpi$ and highest weight vector $\xi_{\varpi}$. Then by induction we see that $\xi_{k}=F_{r_{k}}^{\left(\varpi, \beta_{k}^{\vee}\right)} \cdots F_{r_{1}}^{\left(\varpi, \beta_{1}^{\vee}\right)} \xi_{\varpi}$ has the weight $s_{r_{k}} \cdots s_{r_{1}}(\varpi)$ for $1 \leq k \leq M$. Moreover, for each $0 \leq k<M$, Bou07b, Section VIII.7.2, Proposition 3] implies that $E_{r_{k+1}} \xi_{k}=0$ and that $\left(\varpi, \beta_{k}^{\vee}\right)$ is the largest integer $n$ such that $F_{r_{k+1}}^{n} \xi_{k} \neq 0$ (we put $\left.\xi_{0}=\xi_{\varpi}\right)$. Hence from the formulas in Jan96, Section 8.6] we see that

$$
T_{w_{X}} \xi_{\varpi}=q^{2\left(\varpi, \rho_{X}\right)} \frac{\prod_{k=1}^{M}(-1)^{\left(\varpi, \beta_{k}^{\vee}\right)}}{\prod_{k=1}^{M}\left[\left(\varpi, \beta_{k}^{\vee}\right)\right]_{q_{r_{k}}} !} Z_{\varpi}^{-} \xi_{\varpi}
$$

for the lowest weight vectors, with

$$
Z_{\varpi}^{-}=F_{r_{M}}^{\left(\varpi, \beta_{M}^{\vee}\right)} \cdots F_{r_{1}}^{\left(\varpi, \beta_{1}^{\vee}\right)} .
$$

In particular, the right hand side, hence $Z_{\varpi}^{-} \xi_{\varpi}$ itself as well, does not depend on the particular decomposition of $w_{X}$. The same computations give

$$
T_{w_{X}}^{-1} \xi_{\varpi}=\frac{1}{\prod_{k=1}^{M}\left[\left(\varpi, \beta_{k}^{\vee}\right)\right]_{q_{r_{k}}} !} Z_{\varpi}^{-} \xi_{\varpi} .
$$

Similarly, we put

$$
Z_{w_{X} \omega}^{+}=E_{r_{M}}^{\left(\varpi, \beta_{M}^{\vee}\right)} \cdots E_{r_{1}}^{\left(\varpi, \beta_{1}^{\vee}\right)}
$$

so that $Z_{w_{X} \varpi}^{+}$transports a lowest weight vector of $V_{\varpi}$ to a multiple of $\xi_{\varpi}$ (the multiple again being independent of the chosen reduced expression). Concretely,

$$
\begin{aligned}
& T_{w_{X}}\left(T_{w_{X}} \xi_{\varpi}\right)=\frac{1}{\prod_{k=1}^{M}\left[\left(\varpi, \beta_{k}^{\vee}\right)\right]_{q_{r_{k}}} !} Z_{w_{X} \varpi}^{+}\left(T_{w_{X}} \xi_{\varpi}\right), \\
& T_{w_{X}}^{-1}\left(T_{w_{X}} \xi_{\varpi}\right)=q^{-2\left(\varpi, \rho_{X}\right)} \frac{\prod_{k=1}^{M}(-1)^{\left(\varpi, \beta_{k}^{\vee}\right)}}{\prod_{k=1}^{M}\left[\left(\varpi, \beta_{k}^{\vee}\right)\right]_{q_{r_{k}}} !} Z_{w_{X} \varpi}^{+}\left(T_{w_{X}} \xi_{\varpi}\right) .
\end{aligned}
$$

When $r \in I \backslash X$, the restriction of $w_{X}\left(\alpha_{r}\right)$ to $\mathfrak{h}_{X}$ is a dominant weight Kol14, Section 3.3]. Interpreting $-\alpha_{r}$ and $w_{X}\left(\alpha_{r}\right)$ as dominant weights on $\mathfrak{g}_{X}$, we have

$$
Z_{r}^{-}(X)=Z_{-\alpha_{r}}^{-}, \quad Z_{r}^{+}(X)=Z_{\alpha_{r}}^{+}
$$

in the notation of Kol14, Section 4.3].

Let us denote now by $\operatorname{Ad}_{q}$ the adjoint action of $U_{q}(\mathfrak{g})$ on itself, defined by

$$
\operatorname{Ad}_{q}(x)(y)=x_{(1)} y S\left(x_{(2)}\right)
$$

where $S$ is the antipode of $U_{q}(\mathfrak{g})$ and where we have used the Sweedler notation $\Delta(x)=x_{(1)} \otimes x_{(2)}$. Following still the notation of Kol14, Section 4.3], we write $a_{r}^{+}$for the unique number such that

$$
T_{w_{X}}\left(E_{r}\right)=a_{r}^{+} \operatorname{Ad}_{q}\left(Z_{r}^{+}(X)\right)\left(E_{r}\right) .
$$

It is easy to see that $a_{r}^{+}=a_{\tau(r)}^{+} \in \mathbb{R}$. Be careful, however, that $T_{w_{X}}\left(E_{r}\right)$ is considered as the automorphism $T_{w_{X}}$ applied to $E_{r} \in U_{q}(\mathfrak{g})$, which is not a priori the same as $T_{w_{X}}$ applied to $E_{r}$ considered as an element of the $U_{q}\left(\mathfrak{g}_{X}\right)$-module $\operatorname{Ad}_{q}\left(U_{q}\left(\mathfrak{g}_{X}\right)\right)\left(E_{r}\right)$ under the adjoint action.

Definition B.1. We write $e_{\varpi}, d_{w_{X} \varpi} \in \mathbb{R}$ for the unique numbers such that in $V_{\varpi}$ we have

$$
Z_{w_{X} \varpi}^{+} Z_{\varpi}^{-} \xi_{\varpi}=e_{\varpi} \xi_{\varpi}, \quad Z_{\varpi}^{-} Z_{w_{X} \varpi}^{+} T_{w_{X}} \xi_{\varpi}=d_{w_{X} \varpi} T_{w_{X}} \xi_{\varpi} .
$$

The following lemma follows immediately from (B.1) and B.3.

Lemma B.2. We have

$$
e_{\varpi}=d_{w_{X} \varpi}=\prod_{k=1}^{M}\left(\left[\left(\varpi, \beta_{k}^{\vee}\right)\right]_{q_{r_{k}}} !\right)^{2} .
$$


Lemma B.3. For $r \in I \backslash X$, we have $a_{r}^{+}=d_{\alpha_{r}}^{-1 / 2}$.

Proof. Let $\varpi_{r}$ be the fundamental weight of $\mathfrak{g}$ dual to $\alpha_{r}$, and let $w_{0}$ be the longest element of the Weyl group of $\mathfrak{g}$. Then $-w_{0} \varpi_{r}$ is a dominant integral weight for $\mathfrak{g}$ such that the $U_{q}(\mathfrak{g})$-module $V_{-w_{0} \varpi_{r}}$ has a lowest weight vector $\eta_{-\varpi_{r}}$ at weight $-\varpi_{r}$. It follows that

$$
T_{w_{X}}\left(E_{r}\right) \eta_{-\varpi_{r}}=T_{w_{X}}\left(E_{r}\right) T_{w_{X}}\left(\eta_{-\varpi_{r}}\right)=T_{w_{X}}\left(E_{r} \eta_{-\varpi_{r}}\right) .
$$

On the other hand, from a concrete formula of $S$ and that $\eta_{-\varpi_{r}}$ is a lowest weight vector, we have

$$
T_{w_{X}}\left(E_{r}\right) \eta_{-\varpi_{r}}=a_{r}^{+} \operatorname{Ad}_{q}\left(Z_{\alpha_{r}}^{+}\right)\left(E_{r}\right) \eta_{-\varpi_{r}}=a_{r}^{+} Z_{\alpha_{r}}^{+} E_{r} \eta_{-\varpi_{r}}
$$

Now $E_{r} \eta_{-\varpi_{r}}$ spans an $U_{q}\left(\mathfrak{g}_{X}\right)$-module isomorphic to the highest weight-module $V_{w_{X} \alpha_{r}}$ of $U_{q}\left(\mathfrak{g}_{X}\right)$, where under this correspondence $E_{r} \eta_{-\varpi_{r}}$ is sent to a lowest weight vector $Z_{w_{X} \alpha_{r}}^{-} \xi_{w_{X} \alpha_{r}}$. Then it follows from (B.2) that

while by definition

$$
T_{w_{X}} Z_{w_{X} \alpha_{r}}^{-} \xi_{w_{X} \alpha_{i}}=\prod_{k=1}^{M}\left(\left[\left(w_{X} \alpha_{r}, \beta_{k}^{\vee}\right)\right]_{q_{r_{k}}} !\right) \xi_{w_{X} \alpha_{r}}
$$

This finishes the proof.

$$
a_{r}^{+} Z_{\alpha_{r}}^{+} Z_{w_{X} \alpha_{r}}^{-} \xi_{w_{X} \alpha_{r}}=a_{r}^{+} e_{w_{X} \alpha_{r}} \xi_{w_{X} \alpha_{r}}
$$

We are now ready to determine the $*$-invariance of $U_{q}^{\mathbf{t}}\left(\mathfrak{k}^{\mathbb{C}}\right)$. We start with two easy lemmas, the first of which is implicit in Kol14.

Lemma B.4. For all $r \in I$, we have

$$
K_{\tau(r)} K_{r}^{-1} \in U_{q}^{\mathbf{t}}\left(\mathfrak{k}^{\mathbb{C}}\right)
$$

Proof. Since $\Theta=-w_{X} \circ \tau$, we have

$$
\alpha_{\tau(r)}-\alpha_{r}=\left(\alpha_{\tau(r)}-w_{X} \alpha_{\tau(r)}\right)-\left(\Theta\left(\alpha_{r}\right)+\alpha_{r}\right)
$$

with $\alpha_{\tau(r)}-w_{X} \alpha_{\tau(r)} \in \mathbb{Z} X$, while $\Theta\left(\alpha_{r}\right)+\alpha_{r}$ is $\Theta$-invariant.

Lemma B.5. If $x \in U_{q}^{\mathbf{t}}\left(\mathfrak{k}^{\mathbb{C}}\right), r \in I$ and $y \in U_{q}\left(\mathfrak{g}_{X}\right)$, then

$$
\operatorname{Ad}_{q}(y)\left(x K_{\tau(r)}\right) K_{r}^{-1} \in U_{q}^{\mathbf{t}}\left(\mathfrak{k}^{\mathbb{C}}\right) .
$$

Proof. We have

$$
\operatorname{Ad}_{q}(y)\left(x K_{\tau(r)}\right) K_{r}^{-1}=\operatorname{Ad}_{q}\left(y_{(1)}\right)(x) \operatorname{Ad}_{q}\left(y_{(2)}\right)\left(K_{\tau(r)}\right) K_{r}^{-1} .
$$

However, $\operatorname{Ad}_{q}(c)(x) \in U_{q}^{\mathbf{t}}\left(\mathfrak{k}^{\mathbb{C}}\right)$ for all $c \in U_{q}\left(\mathfrak{g}_{X}\right)$, while

$$
\operatorname{Ad}_{q}\left(U_{q}\left(\mathfrak{g}_{X}\right)\right)\left(K_{\tau(r)}\right) K_{r}^{-1} \in U_{q}\left(\mathfrak{g}_{X}\right) K_{\tau(r)} U_{q}\left(\mathfrak{g}_{X}\right) K_{r}^{-1}=U_{q}\left(\mathfrak{g}_{X}\right) K_{\tau(r)} K_{r}^{-1} \subseteq U_{q}^{\mathbf{t}}\left(\mathfrak{k}^{\mathbb{C}}\right) .
$$

This proves the assertion.

We are now ready to prove Theorem 3.11. In the proof of the following, we use again the shorthand

$$
z_{r}=s(X, \tau)\left(\alpha_{r}\right)
$$

under the notation of Kol14].

Theorem B.6. The algebra $U_{q}^{\mathbf{t}}\left(\mathfrak{k}^{\mathbb{C}}\right)$ is $*$-invariant if $s_{r} \in i \mathbb{R}$ and

$$
c_{\tau(r)} c_{r}=q^{\left(\Theta\left(\alpha_{r}\right)-\alpha_{r}, \alpha_{\tau(r)}\right)}
$$

for all $r \in I \backslash X$.

Proof. Clearly $U_{q}\left(\mathfrak{g}_{X}\right)$ and $U\left(\mathfrak{h}^{\theta}\right)$ are $*$-invariant. We are to show that $B_{r}^{*} \in U_{q}^{\mathbf{t}}\left(\mathfrak{k}^{\mathbb{C}}\right)$ for $r \in I \backslash X$.

Recall first that $F_{r} K_{r}$ spans an irreducible $\operatorname{Ad}_{q}\left(U_{q}\left(\mathfrak{g}_{X}\right)\right)$-module Kol14, Lemma 3.5]. We consider now two cases.

Suppose first that the $\operatorname{Ad}_{q}\left(U_{q}\left(\mathfrak{g}_{X}\right)\right)$-module spanned by $F_{r} K_{r}$ is one-dimensional, which is equivalent with $a_{r s}=0$ for all $s \in X$. Then also the $\operatorname{Ad}_{q}\left(U_{q}\left(\mathfrak{g}_{X}\right)\right)$-module spanned by $E_{r}$ is one-dimensional. In particular, it follows that $T_{w_{X}}\left(E_{r}\right)=E_{r}$, and hence, since $z_{r}=1$ as $a_{r s}=0$ for all $s \in X$,

$$
\theta_{q}\left(F_{r} K_{r}\right)=-E_{\tau(r)},
$$

so

$$
B_{r}=F_{r}-c_{r} E_{\tau(r)} K_{r}^{-1}+s_{r} K_{r}^{-1}
$$


Then

$$
B_{r}^{*}=K_{r}^{-1} E_{r}-\overline{c_{r}} K_{r}^{-1} F_{\tau(r)} K_{\tau(r)}+\overline{s_{r}} K_{r}^{-1} .
$$

On the other hand, since $K_{r}^{-1} K_{\tau(r)} \in U_{q}\left(\mathfrak{h}^{\theta}\right) U_{q}\left(\mathfrak{g}_{X}\right)$, we have

$$
K_{r}^{-1} K_{\tau(r)} B_{\tau(r)}=K_{r}^{-1} K_{\tau(r)} F_{\tau(r)}-c_{\tau(r)} K_{r}^{-1} K_{\tau(r)} E_{r} K_{\tau(r)}^{-1}+s_{\tau(r)} K_{r}^{-1} \in U_{q}^{\mathbf{t}}\left(\mathfrak{k}^{\mathbb{C}}\right) .
$$

Hence $B_{r}^{*} \in U_{q}^{\mathbf{t}}\left(\mathfrak{k}^{\mathbb{C}}\right)$ if

$$
\left(1-\overline{c_{r}} c_{\tau(r)} q_{r}^{2} q^{\left(\alpha_{r}, \alpha_{\tau(r)}\right)}\right) K_{r}^{-1} E_{r}+\left(\overline{s_{r}}+q_{r}^{2} \overline{c_{r}} s_{\tau(r)}\right) K_{r}^{-1} \in U_{q}^{\mathbf{t}}\left(\mathfrak{k}^{\mathbb{C}}\right) .
$$

Hence it is sufficient that

$$
\overline{c_{r}} c_{\tau(r)} q^{\left(\alpha_{r}, \alpha_{\tau(r)}+\alpha_{r}\right)}=1, \quad \overline{s_{r}}=-q_{r}^{2} \overline{c_{r}} s_{\tau(r)} .
$$

Since our assumption implies $w_{X} \alpha_{r}=\alpha_{r}$, it is easily seen that $c_{\tau(r)} \overline{c_{r}}$ must have the form as in the statement of the proposition, since in this case $a_{r}^{+}=1$ and $\Theta\left(\alpha_{r}\right)=-w_{X} \tau\left(\alpha_{r}\right)=-\alpha_{\tau(r)}$. Moreover, if $s_{r} \neq 0$, then we are necessarily in the situation where $\tau(r)=r$. Since we assume that $c_{r}>0$ in this case, we obtain then $c_{r}=q_{r}^{-2}$, and it follows that $s_{r}$ is purely imaginary.

Let us now consider the case where $a_{r s} \neq 0$ for some $s \in X$. In particular, this automatically forces $s_{r}=0$. Let us use the notation $v_{r}$ as in [Kol14. Theorem 4.4.(3)], where we note that

$$
v_{r}=s(X, \tau)\left(w_{X} \alpha_{\tau(r)}\right) a_{r}^{+}=\overline{z_{r}} a_{r}^{+} .
$$

An easy computation, using that $\operatorname{Ad}_{q}(y)(x)^{*}=\operatorname{Ad}_{q}\left(S(y)^{*}\right)\left(x^{*}\right)$, shows

$$
B_{r}^{*}=K_{r}^{-1} E_{r}-\overline{c_{r} v_{r}} K_{r}^{-1} \operatorname{Ad}_{q}\left(S\left(Z_{\tau(r)}^{+}\right)^{*}\right)\left(F_{\tau(r)} K_{\tau(r)}\right) .
$$

On the other hand, it follows from Lemma B.5 that

$$
\operatorname{Ad}_{q}\left(S\left(Z_{\tau(r)}^{+}(X)\right)^{*}\right)\left(B_{\tau(r)} K_{\tau(r)}\right) K_{r}^{-1} \in U_{q}^{\mathbf{t}}\left(\mathfrak{k}^{\mathbb{C}}\right) .
$$

Up to a scalar, this is the same element as

$$
\begin{aligned}
K_{r}^{-1} \operatorname{Ad}_{q}\left(S\left(Z_{\tau(r)}^{+}\right)^{*}\right)\left(K_{r} B_{\tau(r)} K_{\tau(r)} K_{r}^{-1}\right) & \\
=q^{-\left(\alpha_{r}, \alpha_{\tau(r)}\right)} & K_{r}^{-1} \operatorname{Ad}_{q}\left(S\left(Z_{\tau(r)}^{+}\right)^{*}\right)\left(F_{\tau(r)} K_{\tau(r)}\right) \\
& +q^{-\left(\Theta\left(\alpha_{r}\right), \alpha_{\tau(r)}\right)} c_{\tau(r)} K_{r}^{-1} \operatorname{Ad}_{q}\left(S\left(Z_{\tau(r)}^{+}\right)^{*}\right)\left(\theta_{q}\left(F_{\tau(r)} K_{\tau(r)}\right)\right)
\end{aligned}
$$

Now note that $S\left(Z_{\tau(r)}^{+}(X)\right)^{*}=(-1)^{2\left(\rho_{X}^{\vee}, \alpha_{r}\right)} Z_{\tau(r)}^{-}(X)$. Since

$$
\operatorname{Ad}_{q}\left(Z_{\tau(r)}^{-}(X) Z_{r}^{+}(X)\right)\left(E_{r}\right)=\operatorname{Ad}_{q}\left(Z_{-\alpha_{\tau(r)}}^{-} Z_{\alpha_{r}}^{+}\right)\left(E_{r}\right)=\operatorname{Ad}_{q}\left(Z_{w_{X} \alpha_{r}}^{-} Z_{\alpha_{r}}^{+}\right)\left(E_{r}\right)=d_{\alpha_{r}} E_{r},
$$

we see that $B_{r}^{*} \in U_{q}^{\mathbf{t}}\left(\mathfrak{k}^{\mathbb{C}}\right)$ if

$$
c_{\tau(r)} \overline{c_{r}} v_{\tau(r)} \overline{v_{r}}=(-1)^{2\left(\rho_{X}^{\vee}, \alpha_{r}\right)} q^{\left(\Theta\left(\alpha_{r}\right)-\alpha_{r}, \alpha_{\tau(r)}\right)} d_{\alpha_{r}}^{-1} .
$$

However, the conditions on an admissible pair imply that

$$
z_{r} \overline{z_{\tau(r)}}=z_{r}^{2}=(-1)^{2\left(\rho_{X}^{\vee}, \alpha_{r}\right)} .
$$

Using (B.4), we can then simplify the above identity, using Lemma B.3, to the one in the statement of the proposition.

\section{Appendix C. Characters of Letzter-Kolb Coideals}

Let $\theta=\theta(X, \tau)$ for $(X, \tau)$ an admissible pair, and put $\mathfrak{k}=\mathfrak{u}^{\theta}$. We assume that $\mathfrak{k} \subseteq \mathfrak{u}$ is an irreducible symmetric pair. One then has a non-trivial character on $\mathfrak{k}=\mathfrak{u}^{\theta}$ if and only if $\mathfrak{k} \subseteq \mathfrak{u}$ is a Hermitian symmetric pair. In particular, $\mathfrak{u}$ is simple and the space of characters on $\mathfrak{k}$ is one-dimensional. We will show that, analogously, the parameters $\mathbf{c}, \mathbf{s}$ can be non-trivial only in the Hermitian symmetric case, and then they are determined by a single parameter.

Definition C.1. We say that a symmetric pair $\mathfrak{k}=\mathfrak{u}^{\theta} \subseteq \mathfrak{u}$ is;

- of $S$-type if $I_{\mathcal{S}} \neq \emptyset$,

- of $C$-type if there exist $i \in I \backslash X$ with $\tau(i) \neq i$ but $i \notin I_{\mathcal{C}}$; thus, $\mathfrak{g}_{2 \overline{\alpha_{i}}} \neq 0$.

Lemma C.2. A symmetric pair is of $S$-or C-type if and only if it is Hermitian, in which case it can not be both. Moreover, if the symmetric pair is of $S$-type, there is exactly one element in $I_{\mathcal{S}}$, while if it is of $C$-type, there is exactly one two-element $\tau$-orbit $\{i, \tau(i)\}$ in $I \backslash X$ with $i \notin I_{\mathcal{C}}$. 
For $\mathfrak{k} \subseteq \mathfrak{u}$ of Hermitian type, we will call a simple root $\alpha_{i}$ with $i \in I^{*}$ distinguished if $\alpha_{i} \in I_{\mathcal{S}}$ (for S-type) or $\tau(i) \neq i$ but $i \notin I_{\mathcal{C}}$ (for C-type).

Proof. This follows by a type-by-type verification, see also the discussion below.

In the following, we will list the S-type and C-type Hermitian symmetric pairs $(\mathfrak{k} \subseteq \mathfrak{u})$ with the $\tau$-orbit of their distinguished simple root as in the statement of the lemma, using the numbering of roots as in OV90, Reference Chapter, table 9] (note that $\mathfrak{s p}_{l}$ is a compact real form of $\mathfrak{s p}_{2 l}(\mathbb{C})$ ):

(1) S-type:

- AIII $\left(\mathfrak{s}\left(\mathfrak{u}_{p} \oplus \mathfrak{u}_{p}\right) \subseteq \mathfrak{s u}_{2 p}\right.$ for $\left.p \geq 1\right): \alpha_{p}$.

- DIII $\left(\mathfrak{u}_{2 p} \subseteq \mathfrak{s o}_{4 p}\right.$ for $\left.p \geq 1\right): \alpha_{2 p}$.

- $\mathrm{BDI}\left(\mathfrak{s o}_{2} \oplus \mathfrak{s o}_{q} \subseteq \mathfrak{s o}_{2+q}\right.$ for $\left.q \geq 3\right): \alpha_{1}$.

- CI $\left(\mathfrak{u}_{l} \subseteq \mathfrak{s p}_{l}\right.$ with $\left.l \geq 2\right): \alpha_{l}$.

- EVII $\left(\mathfrak{e}_{6} \oplus \mathfrak{u}_{1} \subseteq \mathfrak{e}_{7}\right): \alpha_{1}$.

(2) C-type:

- AIII $\left(\mathfrak{s}\left(\mathfrak{u}_{p} \oplus \mathfrak{u}_{q}\right) \subseteq \mathfrak{s u}_{p+q}\right.$ with $\left.p<q\right):\left\{\alpha_{p}, \alpha_{q}\right\}$.

- DIII $\left(\mathfrak{u}_{2 p+1} \subseteq \mathfrak{s o}_{4 p+2}\right.$ with $\left.p \geq 2\right):\left\{\alpha_{2 p}, \alpha_{2 p+1}\right\}$.

- EIII $\left(\mathfrak{s o}_{10} \oplus \mathfrak{u}_{1} \subseteq \mathfrak{e}_{6}\right):\left\{\alpha_{1}, \alpha_{5}\right\}$.

In the C-type case, we will take resp. $\alpha_{p}, \alpha_{2 p+1}$ and $\alpha_{1}$ as the distinguished vector. This way the distinguished vector is always the noncompact simple positive root in the conventional presentation of inclusion $\mathfrak{k} \subseteq \mathfrak{u}$, cf. Kna02, Appendix $\mathrm{C}]{ }^{11}$

Corollary C.3. Let $U_{q}^{\mathfrak{t}}(\mathfrak{k}) \subseteq U_{q}(\mathfrak{u})$ be a coideal $*$-subalgebra associated to $\mathfrak{k}=\mathfrak{u}^{\theta} \subseteq \mathfrak{u}$, with all $c_{r}>0$.

(1) If $\mathfrak{k} \subseteq \mathfrak{u}$ is not of Hermitian type, then $s_{r}=0$ and for all $r \in I \backslash X$

$$
c_{r}=q^{\frac{1}{2}\left(\Theta\left(\alpha_{r}\right)-\alpha_{\tau(r)}, \alpha_{r}\right)} .
$$

(2) If $\mathfrak{k} \subseteq \mathfrak{u}$ is of Hermitian type S-type, then $s_{r}=0$ for $r$ not distinguished and for all $r \in I \backslash X$

$$
c_{r}=q^{\frac{1}{2}\left(\Theta\left(\alpha_{r}\right)-\alpha_{\tau(r)}, \alpha_{r}\right)} .
$$

At the distinguished vector one has $s_{r} \in i \mathbb{R}$, and all values are allowed.

(3) If $\mathfrak{k} \subseteq \mathfrak{u}$ is of Hermitian type C-type, then $s_{r}=0$ for all $r \in I \backslash X$ and for all $r \in I^{*}$

$$
c_{r}=c_{\tau(r)}=q^{\frac{1}{2}\left(\Theta\left(\alpha_{\tau(r)}\right)-\alpha_{r}, \alpha_{\tau(r)}\right)}
$$

except possibly at the distinguished vertex $r$ where one has

$$
c_{r}=q^{\frac{1}{2}\left(\Theta\left(\alpha_{r}\right)-\alpha_{\tau(r)}, \alpha_{r}\right)} q^{\lambda}, \quad c_{\tau(r)}=q^{\frac{1}{2}\left(\Theta\left(\alpha_{r}\right)-\alpha_{\tau(r)}, \alpha_{i}\right)} q^{-\lambda}
$$

for some $\lambda \in \mathbb{R}$. Any value of $\lambda$ can occur.

We will show now, in fact, that the parameter case can be obtained in a simple way from the nonparameter case. For this, we will study the set of $*$-characters on the coideals.

Definition C.4. If $B$ is a right coideal $*$-subalgebra and $\chi$ a $*$-character on $B$, we call

$$
B^{\prime}=(\chi \otimes \mathrm{id}) \Delta(B)
$$

the $\chi$-conjugate of $B$.

It is clear that $B^{\prime}$ is again a coideal $*$-subalgebra. Our aim is to show now that any two coideal subalgebras $U_{q}^{\mathbf{t}}(\mathfrak{k}), U_{q}^{\mathbf{t}^{\prime}}(\mathfrak{k})$ corresponding to the same symmetric pair are $\chi$-conjugate for some $\chi$. We start by determining the relations for the maximal commutative quotient of $U_{q}^{\mathrm{t}}$. In the following lemma, we write

$$
J=\left\{r \in I \backslash X \mid \alpha_{r} \perp \alpha_{s} \text { for all } s \in X\right\} .
$$

We will only determine the relations in the case all $\left|a_{r s}\right| \leq 2$, as this is the only case of interest for us.

In the following we denote the generators $F_{r}$ of $U_{q}^{\mathbf{t}}(\mathfrak{k})$ for $r \in X$ by $B_{r}$.

\footnotetext{
${ }^{11}$ Note that the above list of cases with non-trivial parameters can also be found in Let03, however the case of $B D I$ (corresponding to the two cases $B I$ and $D I$ ) is erronously listed as never having parameters.
} 
Lemma C.5. Let $C_{\mathbf{t}}$ be the maximal commutative quotient of $U_{q}^{\mathbf{t}}(\mathfrak{k})$, and write $\bar{Z}$ for the images of the generators $Z$ of $U_{q}^{\mathbf{t}}(\mathfrak{k})$ in $C_{\mathbf{t}}$. Then $C_{\mathbf{t}}$ is a universal commutative algebra with generators $\bar{Z}$ and the following relations:

- $\bar{K}_{\omega+\omega^{\prime}}=\bar{K}_{\omega} \bar{K}_{\omega^{\prime}}$ for $\omega, \omega^{\prime} \in P^{\Theta}$.

- $\bar{K}_{r}^{2}=1$ for $r \in X$.

- $\bar{E}_{r}=\bar{B}_{r}=0$ for $r \in X$.

- $\bar{B}_{r}=0$ for $\Theta\left(\alpha_{r}\right) \neq-\alpha_{r}$.

- For $a_{r, \tau(r)}=0$,

$$
\delta_{r \in J}\left(c_{r} \bar{K}_{\alpha_{\tau(r)}-\alpha_{r}}^{2}-c_{\tau(r)}\right)=0 .
$$

- For $a_{r s}=-1$,

$$
\begin{aligned}
\left(1-q_{r}\right)\left(1-q_{r}^{-1}\right) \bar{B}_{r}^{2} \bar{B}_{s}=- & \delta_{r \in J} \delta_{r, \tau(r)} q_{r} c_{r} \bar{K}_{\alpha_{\tau(r)}-\alpha_{r}} \bar{B}_{s} \\
& +\delta_{r, \tau(s)}\left(q_{r}+q_{r}^{-1}\right)\left(\delta_{s \in J} q_{r} c_{s} \bar{K}_{\alpha_{\tau(s)}-\alpha_{s}}+\delta_{r \in J} q_{r}^{-2} c_{r} \bar{K}_{\alpha_{\tau(r)}-\alpha_{r}}\right) \bar{B}_{r}
\end{aligned}
$$

- For $a_{r, \tau(r)}=-2$,

$$
\left(q_{r}^{-8} c_{r} \bar{K}_{\alpha_{\tau(r)}-\alpha_{r}}-c_{\tau(r)}\right) \bar{B}_{r}=0 .
$$

If furthermore $D_{\mathbf{t}}$ is the maximal commutative $*$-algebra quotient, then the extra relations in $D_{\mathbf{t}}$ are

- $\bar{B}_{r}=0$ for $r \notin J$.

- For $r \in J$,

$$
\bar{B}_{r}^{*}=-c_{\tau(r)}^{-1} q^{-\left(\alpha_{r}, \alpha_{\tau(r)}\right)} \bar{K}_{\alpha_{\tau(r)}-\alpha_{r}} \bar{B}_{\tau(r)} .
$$

Proof. The first two relations are immediate, since we can use the ordinary quantum group relations of $U_{q}\left(\mathfrak{g}_{X}\right)$. Also the third relation is immediate from Kol14, Theorem 7.1], as we have non-trivial $q$-commutation relations between $U_{q}\left(\mathfrak{h}^{\theta}\right)$ and $B_{r}$ when $r \notin J$.

For the remainder of the relations, we claim that the elements $\mathcal{Z}_{r}=0$, introduced just before Kol14, Lemma 7.2], vanish in the quotient, except when $r \in J$. Indeed, by its definition $\mathcal{Z}_{r}$ is a linear combination of elements of the form $E_{r_{1}} \ldots E_{r_{p}} K_{\alpha_{\tau(r)}-\alpha_{r}} K_{\gamma}$ for $\gamma \in \mathbb{Z} X$ variable and some fixed $p$, which vanishes in the quotient unless $p=0$. But $p=0$ only when $\alpha_{r}$ is fixed under all $s_{r^{\prime}}$ for $r^{\prime} \in X$, i.e., when $r \in J$.

Similarly, we claim that the elements $\mathcal{W}_{r s}$, introduced in Kol14, Lemma 7.7], disappear in the quotient for all $r \in I \backslash X$ and $s \in X$. Indeed, we have again by definition that $\mathcal{W}_{r s}$ is a linear combination of elements of the form $E_{s_{1}} \ldots E_{s_{p}} K_{\gamma}$ for $\gamma \in \mathbb{Z} X$ variable and some fixed $p$. This does not vanish in the quotient only if $p=0$, which is the case precisely when $w_{X}\left(\alpha_{r}\right)=\alpha_{r}+\alpha_{s}$. But this situation does not occur, cf. the proof of [Kol14, Lemma 5.11, Step 1].

The remainder of the relations are now obvious from Kol14, Theorems 7.1, 7.4 and 7.8]. Indeed, the relations for $r \in I \backslash X$ and $s \in X$ all vanish, while the relations for $r, s \in I \backslash X$ follow from the observation that

$$
\sum_{n}(-1)^{n}\left[\begin{array}{c}
m \\
n
\end{array}\right]_{q}=\left(1-q^{m-1}\right)\left(1-q^{m-3}\right) \ldots\left(1-q^{-m+1}\right) .
$$

If we now demand that also the $*$-structure passes to the quotient, then, as follows from the proof of Theorem B.6. the element $B_{r}^{*}$ for $r \notin J$ coincides up to a scalar factor with

$$
K_{r}^{-1} \operatorname{Ad}_{q}\left(S\left(Z_{\tau(r)}^{+}\right)^{*}\right)\left(K_{r} B_{\tau(r)} K_{\alpha_{\tau(r)}-\alpha_{r}}\right),
$$

which shows that necessarily $\bar{B}_{r}=0$. On the other hand, for $r \in J$ it follows immediately that

$$
\bar{B}_{r}^{*}=-c_{\tau(r)}^{-1} q^{-\left(\alpha_{r}, \alpha_{\tau(r)}\right)} \bar{K}_{\alpha_{\tau(r)}-\alpha_{r}} \bar{B}_{\tau(r)} .
$$

This completes the proof.

We can now determine for each Hermitian symmetric space a family of $*$-characters as follows.

Corollary C.6. On each $U_{q}^{\mathbf{t}}(\mathfrak{k})$ associated to a Hermitian symmetric space, one has a one-parameter family of $*$-characters $\chi_{t}, t \in \mathbb{R}$, determined as follows:

- For $S$-type: $\chi_{t}\left(K_{\omega}\right)=1$ for all $\omega \in P^{\Theta}$ and $\chi_{t}\left(B_{r}\right)=0$ for all $r$ except for the distinguished vertex $p$ where $\chi_{t}\left(B_{p}\right)=i t$. 
- For C-type: $\chi_{t}\left(B_{r}\right)=0$ for all $r$ and $\chi_{t}\left(K_{\omega}\right)=q^{f(\omega)}$ for all $\omega \in P^{\Theta}$, where $f$ is the unique linear functional on $\mathfrak{h}^{*}$ which vanishes on the roots in $X$ and is such that $f\left(\alpha_{r}\right)=0$ for all $r \in I \backslash X$ except for the distinguished vertex $p$ where $f\left(\alpha_{p}\right)=t$.

Proof. Note that a vertex $r \in I \backslash X$ satisfies both $r \in J$ and $\Theta\left(\alpha_{r}\right)=-\alpha_{r}$ precisely when $r \in I_{\text {ns }}$. It follows that every *-character $\chi$ of $U_{q}^{\mathbf{t}}(\mathfrak{k})$ vanishes on all generators of $U_{q}^{\mathbf{t}}(\mathfrak{k})$ except possibly for $K_{\omega}$, with $\omega \in P^{\Theta}$, and $B_{r}$, with $r \in I_{\mathrm{ns}}$. We will consider only characters such that $\chi\left(K_{\omega}\right)=q^{f(\omega)}$ for a linear functional $f$ which is real on $P^{\Theta}$ and vanishes on $X$. The values of such an $f$ on $P^{\Theta}$ are determined by $f\left(\alpha_{\tau(r)}-\alpha_{r}\right)$ for $r \in I \backslash X, \tau(r) \neq r$. These values and the numbers $\chi\left(B_{r}\right)\left(r \in I_{\mathrm{ns}}\right)$ must satisfy the relations following from (C.1)- C.3).

In the S-type case, the distinguished vertex $p$ is the unique element of $I_{\mathcal{S}}$ and we have $c_{r}=c_{\tau(r)}$ for all $r \in I \backslash X$. It follows that if we let $f=0$ and $\chi\left(B_{r}\right)=0$ for all nondistinguished vertices in $I_{\mathrm{ns}}$, then the relations (C.1) and C.2 will be satisfied and will impose no restrictions on $\chi\left(B_{p}\right)$ (since by the definition of $I_{\mathcal{S}}$ there is no vertex $r \in I$ such that $a_{r p}=-1$ and $\left.\delta_{r \in J} \delta_{r, \tau(r)}=1\right)$. On the other hand, relation C.3 gives $\chi\left(B_{p}^{*}\right)=-\chi\left(B_{p}\right)$, so $\chi\left(B_{p}\right)$ can be any purely imaginary number.

In the C-type case, if we let $\chi\left(B_{r}\right)=0$ for all $r \in I_{\mathrm{ns}}$, then the only restrictions on $f$ will be given by C.1. If $r \in J$ and $a_{r, \tau(r)}=0$, then $r \in I_{\mathcal{C}}$, so C.1 will be satisfied if we let $f\left(\alpha_{\tau(r)}-\alpha_{r}\right)=0$ for all $r \in I_{\mathcal{C}}$. The complement in $I \backslash X$ of $I_{\mathcal{C}}$ and the vertices fixed by $\tau$ consists precisely of the $\tau$-orbit of the distinguished vertex $p$. Therefore to define $f$ on $P^{\Theta}$ it remains to define its value on $\alpha_{\tau(p)}-\alpha_{p}$, and we can take any real number as this value.

We can now prove the following theorem.

Theorem C.7. All $U_{q}^{\mathbf{t}}(\mathfrak{k})$ associated to the same symmetric pair are $\chi$-conjugate for some $*$-character $\chi$.

Proof. We can of course restrict to the Hermitian symmetric pairs. Assume first that we are in the S-type case, with distinguished vertex $p$. Then if $\chi_{t}$ is one of the $*$-characters on $U_{q}^{\mathbf{t}}(\mathfrak{k})$ as determined above, it follows from

$$
\Delta\left(B_{r}\right)-B_{r} \otimes K_{r}^{-1}-1 \otimes F_{r} \in U_{q}\left(\mathfrak{g}_{X}\right)^{+} K_{\tau(r)} K_{r}^{-1} \otimes U_{q}(\mathfrak{g})
$$

and the fact that $\chi_{t}$ acts as the counit on $U_{q}\left(\mathfrak{g}_{X}\right)^{+} U_{q}\left(\mathfrak{h}^{\theta}\right)$ that

$$
\left(\chi_{t} \otimes \mathrm{id}\right) \Delta\left(B_{r}\right)=B_{r}+\left(\chi\left(B_{r}\right)-s_{r}\right) K_{r}^{-1} .
$$

But this is precisely adding the value $\left(i t-s_{p}\right) K_{p}^{-1}$ to $B_{p}$ at the distinguished vector, and doing nothing for the $B_{r}$ with $r \neq p$. Also on $U_{q}\left(\mathfrak{g}_{X}\right) U_{q}\left(\mathfrak{h}^{\theta}\right)$ the map $\left(\chi_{t} \otimes \mathrm{id}\right) \Delta$ acts as the identity. Hence

$$
\left(\chi_{t} \otimes \mathrm{id}\right)\left(\Delta\left(U_{q}^{(\mathbf{c}, \mathbf{s})}(\mathfrak{k})\right)\right)=U_{q}^{\left(\mathbf{c}, \mathbf{s}^{\prime}\right)}(\mathfrak{k}),
$$

where $s_{i}^{\prime}=i t$ at the distinguished vector and zero elsewhere. It follows that we obtain all coideal *-subalgebras corresponding to the same symmetric pair.

Assume now that we are in C-type. Then the same reasoning (using that all $s_{r}=0$ in this case and that $\chi_{t}$ acts as $q^{f\left(\alpha_{\tau(r)}-\alpha_{r}\right)}$ times the counit on $\left.U_{q}\left(\mathfrak{g}_{X}\right)^{+} K_{\tau(r)} K_{r}^{-1}\right)$ gives that

$$
\left(\chi_{t} \otimes \mathrm{id}\right) \Delta\left(B_{r}\right)=q^{f\left(\alpha_{\tau(r)}-\alpha_{r}\right)} B_{r}+\left(1-q^{f\left(\alpha_{\tau(r)}-\alpha_{r}\right)}\right) F_{r}, \quad\left(\chi_{t} \otimes \mathrm{id}\right)\left(\Delta\left(K_{\alpha}\right)\right)=q^{f(\alpha)} K_{\alpha} .
$$

Since all $c_{r}$ are necessarily positive, this implies that we get $U_{q}^{\left(\mathbf{c}^{\prime}, \mathbf{0}\right)}(\mathfrak{k})$ where $c_{p}^{\prime}=q^{-t} c_{p}, c_{\tau(p)}^{\prime}=q^{t} c_{\tau(p)}$, and all other $c_{r}=c_{r}^{\prime}=1$. Again, it follows that we obtain all coideal $*$-subalgebras corresponding to the same symmetric pair.

Define $c_{r}=q^{\frac{1}{2}\left(\Theta\left(\alpha_{r}\right)-\alpha_{r}, \alpha_{\tau(r)}\right)}$ and $s_{r}=0$, so that $U_{q}^{0}(\mathfrak{k})=U_{q}^{(\mathbf{c}, \mathbf{s})}(\mathfrak{k})$. Let us write $U_{q}^{t}(\mathfrak{k})=U_{q}^{(\mathbf{c}(t), \mathbf{s}(t))}(\mathfrak{k})$, where

- $\mathbf{c}(t)=\mathbf{c}, \mathbf{s}(t)_{r}=\delta_{r, p} i t$ for $p$ distinguished,

- $\mathbf{c}(t)_{r}=c_{r}$ for $r$ or $\tau(r)$ not distinguished, $\mathbf{c}(t)_{r}=q^{-t} c_{r}, \mathbf{c}(t)_{\tau(r)}=q^{t} c_{r}$ for $r$ distinguished, and $\mathbf{s}(t)_{r}=0$ for all $r$.

Corollary C.8. The maps

$$
\pi_{t}: U_{q}^{0}(\mathfrak{k}) \rightarrow U_{q}^{t}(\mathfrak{k}), \quad X \mapsto\left(\chi_{t} \otimes \mathrm{id}\right) \Delta(X)
$$

are $*$-isomorphisms satisfying

$$
\Delta \circ \pi_{t}=\left(\pi_{t} \otimes \mathrm{id}\right) \circ \Delta .
$$




\section{Appendix D. Cylinder twist}

The notion of cylinder twist and cylinder braiding were introduced in tDHO98. In this section, we clarify the connection between cylinder twists and ribbon braided module categories, complementing the algebraic discussion of the connection in [Kol17, Section 2.3].

Let $(\mathcal{C}, \beta)$ be a braided tensor category, $\sigma$ a braided autoequivalence, and $\mathcal{D}$ a right $\mathcal{C}$-module category. For the sake of simplicity we assume that $\mathcal{C}$ is strict and $\mathcal{D}$ is a strict module category, although this is not an essential restriction.

Definition D.1. We call cylinder $\sigma$-twist $\theta$ at $X \in \mathcal{D}$ any natural isomorphism $\theta: X \odot \sigma(-) \rightarrow X \odot-$ of functors $\mathcal{C} \rightarrow \mathcal{D}$ such that $\theta$ satisfies the cylinder $\sigma$-twist equations:

$$
\begin{aligned}
\theta_{U \otimes V}\left(X \odot\left(\sigma_{2}\right)_{U, V}\right) & =\left(X \odot \beta_{V, U}\right)\left(\theta_{V} \otimes U\right)\left(X \odot \beta_{U, \sigma(V)}\right)\left(\theta_{U} \otimes \sigma(V)\right) \\
& =\left(\theta_{U} \otimes V\right)\left(X \odot \beta_{V, \sigma(U)}\right)\left(\theta_{V} \otimes \sigma(U)\right)\left(X \odot \beta_{\sigma(U), \sigma(V)}\right) .
\end{aligned}
$$

For example, if $\mathcal{D}$ is a ribbon $\sigma$-braided $\mathcal{C}$-module category with $\sigma$-braid $\eta$, then $\eta_{X,-}$ is a cylinder $\sigma$-twist at $X$ for each $X \in \mathcal{D}$, using the ribbon $\sigma$-twist equation and naturality on $X \odot U$.

These equalities can be diagrammatically represented by Figure 3
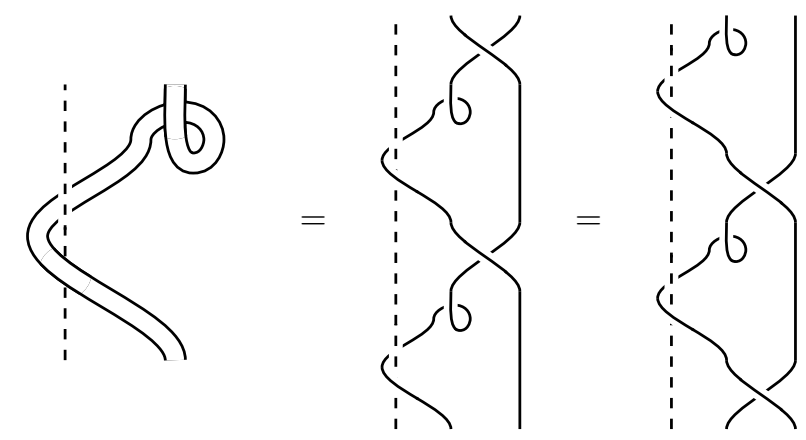

FiguRE 3. cylinder $\sigma$-twist equation

Let us show how one can construct a ribbon $\sigma$-braided module category from a cylinder $\sigma$-twist $\theta$ at $X$ on a right $\mathcal{C}$-module category $\mathcal{D}$. Put

$$
\mathcal{C}_{X}(U, V)=\mathcal{D}(X \odot U, X \odot V)
$$

for objects $U, V$ of $\mathcal{C}$. This defines a right $\mathcal{C}$-module category $\mathcal{C}_{X}$ with the same objects as $\mathcal{C}$, and contains $\mathcal{C}$ as a subcategory. Moreover, $U \mapsto X \odot U$ together with the natural inclusion of morphisms define a functor of module categories from $\mathcal{C}_{X}$ to $\mathcal{D}_{X}$. The only deficit of $\mathcal{C}_{X}$ is that it is not subobject complete, but this can always be amended by passing to the subobject completion.

Put

$$
\eta_{U, V}=\left(X \odot \beta_{V, U}\right)\left(\theta_{V} \odot U\right)\left(X \odot \beta_{U, \sigma(V)}\right): X \odot U \otimes \sigma(V) \rightarrow X \odot U \otimes V,
$$

so $\eta_{U, V} \in \mathcal{C}_{X}(U \otimes \sigma(V), U \otimes V)$. Diagrammatically, we represent $\theta_{V}$ as Figure 4.a and $\eta_{U, V}$ as Figure 4.b.

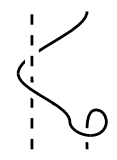

(a) cylinder $\sigma$-twist

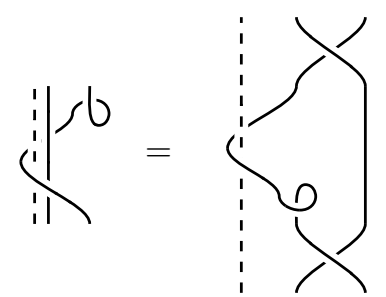

(b) ribbon $\sigma$-braid from cylinder $\sigma$-twist

Figure 4

Proposition D.2. The family $\eta_{U, V}$ above satisfies the $\sigma$-octagon equation and the ribbon $\sigma$-twist equation. 


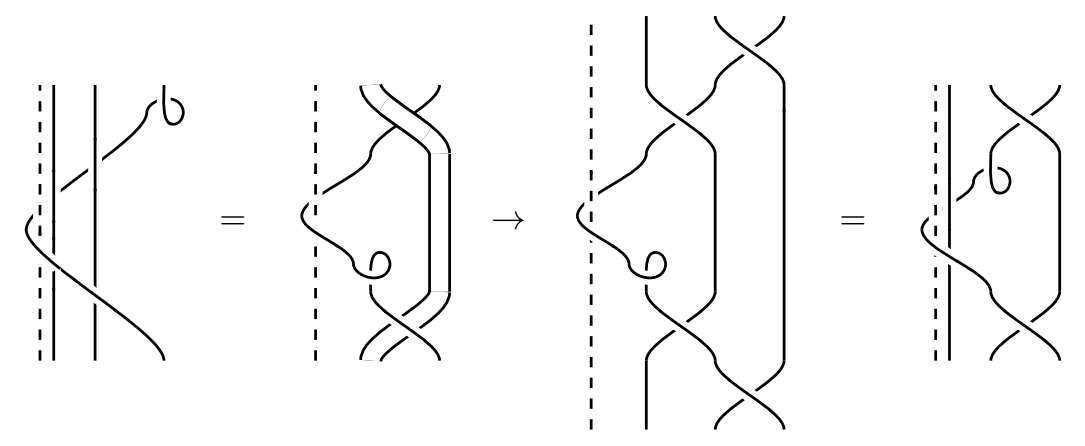

FiguRE 5. $\sigma$-octagon relation

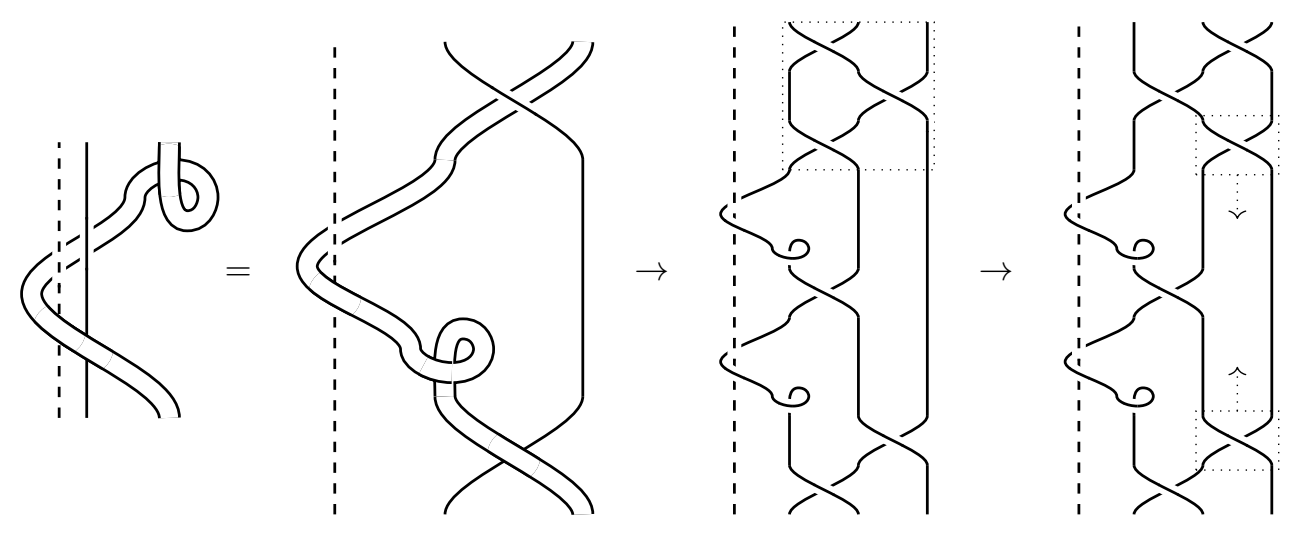

FIGURE 6. ribbon $\sigma$-twist equation

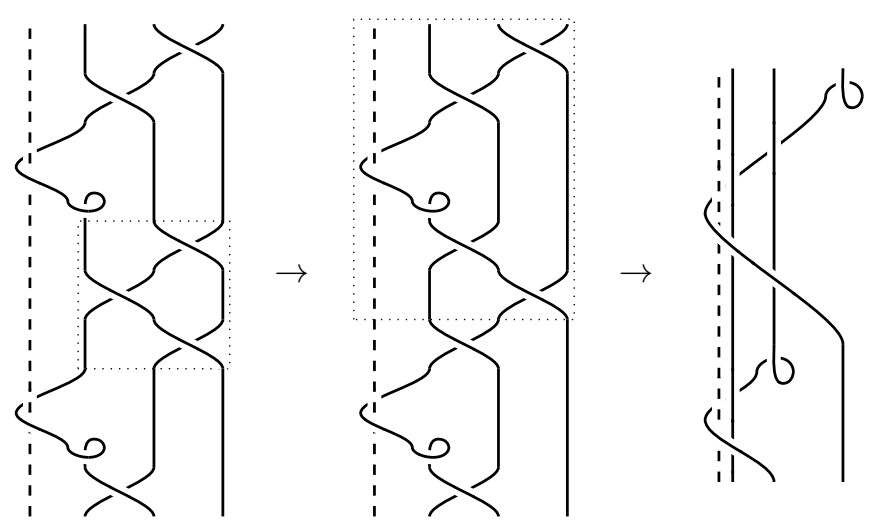

FiguRE 7. ribbon $\sigma$-twist equation, cont.

Proof. The $\sigma$-octagon relation can be verified as in Figure 5, where we used the multiplicativity of $\beta$ such as $\beta_{U \otimes V, W}=\left(\beta_{U, W} \otimes V\right)\left(U \otimes \beta_{V, W}\right)$. For the ribbon $\sigma$-twist relation we manipulate as in Figures 6 and 7. The dotted boxes indicate use of the braid relation, naturality at $U$ in $Y \odot U$, and the $\sigma$-octagon relation (at the final step).

The $\mathcal{C}$-module category $\mathcal{C}_{X}$ will however not necessarily be a ribbon $\sigma$-braided module category, as we do not yet know if $\eta_{U, V}$ is natural in $U$. Therefore, we first restrict the morphisms spaces of $\mathcal{C}_{X}$ as follows:

$$
\tilde{\mathcal{C}}_{X}(U, V)=\left\{f \in \mathcal{D}(X \odot U, X \odot V) \mid \forall W:(f \odot W) \eta_{U, W}=\eta_{V, W}(f \odot \sigma(W))\right\}
$$

This still defines a right $\mathcal{C}$-module category $\tilde{\mathcal{C}}_{X}$ with $\mathcal{C}$ as a subcategory.

Proposition D.3. We have $\eta_{U, V} \in \tilde{\mathcal{C}}_{X}(U \otimes \sigma(V), U \otimes V)$ for any $U$ and $V$. 


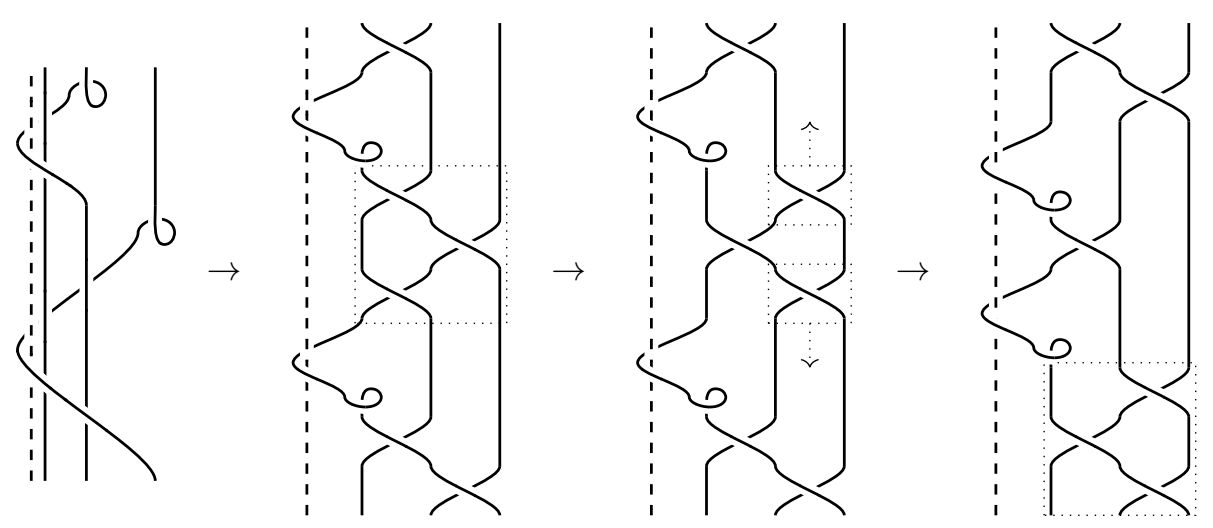

FIGURE 8. naturality

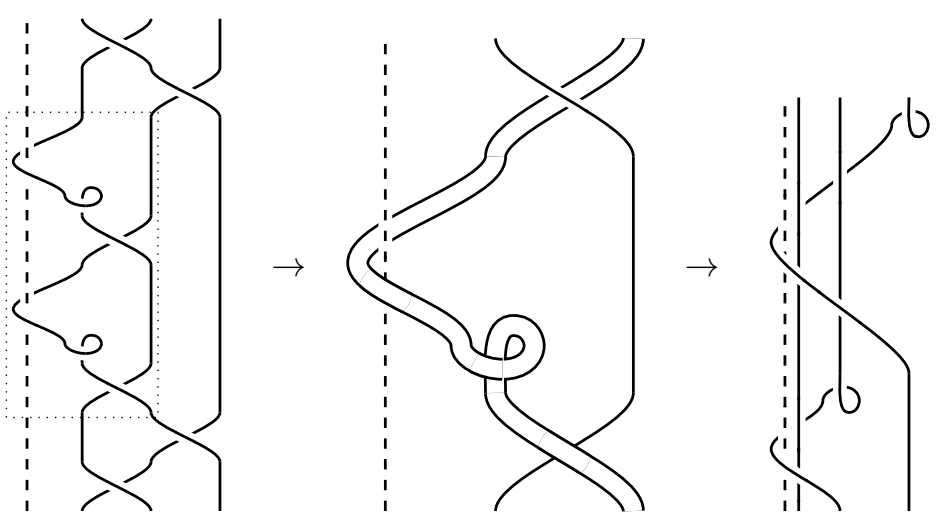

Figure 9. naturality, cont.

Proof. We need to prove $\left(\eta_{U, V} \odot W\right) \eta_{U, W}=\eta_{V, W}\left(\eta_{U, V} \odot \sigma(W)\right)$ for all $W$. The proof goes as in Figures 8 and 9. This time, the dotted boxes represent use of the braid relation, naturality at $U$ in $Y \odot U$, and the cylinder $\sigma$-twist equation for $\theta$ (at the penultimate step). The last step uses the ribbon $\sigma$-twist relation.

From the above, we obtain that $\eta_{U, V} \in \tilde{\mathcal{C}}_{X}(U \otimes V, U \otimes V)$, and the definition of $\tilde{\mathcal{C}}_{X}$ forces $\eta_{U, V}$ to be natural in $U$. It follows that $\tilde{\mathcal{C}}_{X}$ is a ribbon $\sigma$-braided $\mathcal{C}$-module category.

\section{REFERENCES}

$[\mathrm{BV}+95]$ V. Back-Valente, N. Bardy-Panse, H. Ben Messaoud, and G. Rousseau, Formes presque-déployées des algèbres de Kac-Moody: classification et racines relatives, J. Algebra 171 (1995), no. 1, 43-96, DOI : 10.1006/jabr.1995.1004 MR.1314093

[BK15a] M. Balagović and S. Kolb, The bar involution for quantum symmetric pairs, Represent. Theory 19 (2015), 186-210, DOI: 10.1090/ert/469, arXiv:1409.5074 [math.QA]. MR3414769

[BK15b] M. Balagovic and S. Kolb, Universal K-matrix for quantum symmetric pairs, J. Reine Angew. Math, to appear, posted on 2015, DOI:10.1515/crelle-2016-0012, arXiv:1507.06276v2 [math.QA]

[BW18] H. Bao and W. Wang, Canonical bases arising from quantum symmetric pairs, Invent. Math. 213 (2018), no. 3, 1099-1177, DOI:10.1007/s00222-018-0801-5, arXiv:1610.09271 [math.QA], MR3842062

[BZBJ16] D. Ben-Zvi, A. Brochier, and D. Jordan, Quantum character varieties and braided module categories. preprint (2016), available at arXiv:1606.04769 [math.QA], to appear in Sel. Math. New Ser. (2018).

[Bic14] J. Bichon, Hopf-Galois objects and cogroupoids, Rev. Un. Mat. Argentina 55 (2014), no. 2, 11-69, arXiv:1006.3014 [math.QA] MR3285340

[Bou07a] N. Bourbaki. (2007). Éléments de mathématique. Groupes et algèbres de Lie. chapitre 4 à 6, Springer, BerlinHeidelberg, DOI : 10.1007/978-3-540-34491-9 Reprint of the 1981 (Masson) edition.

[Bou07b] N. Bourbaki. (2007). Éléments de mathématique. Groupes et algèbres de Lie. chapitre 7 et 8 , Springer, BerlinHeidelberg, DOI:10.1007/978-3-540-33977-9 Reprint of the 1975 (Hermann) edition.

[Bro12] A. Brochier, A Kohno-Drinfeld theorem for the monodromy of cyclotomic KZ connections, Comm. Math. Phys. 311 (2012), no. 1, 55-96, DOI:10.1007/s00220-012-1424-0, arXiv:1011.4285 [math.QA] MR.2892463

[Bro13] A. Brochier, Cyclotomic associators and finite type invariants for tangles in the solid torus, Algebr. Geom. Topol. 13 (2013), no. 6, 3365-3409, DOI:10.2140/agt.2013.13.3365, arXiv:1209.0417 [math.QA] MR.3248737 
[CP95] V. Chari and A. Pressley. (1995). A guide to quantum groups, Cambridge University Press, Cambridge, ISBN 0-521-55884-0. Corrected reprint of the 1994 original. MR.1358358 (96h:17014)

[Che84] I. V. Cherednik, Factorizing particles on a half line, and root systems, Teoret. Mat. Fiz. 61 (1984), no. 1, 35-44, DOI : 10.1007/BF01038545 MR774205

[Che89] I. V. Cherednik, Generalized braid groups and local r-matrix systems, Dokl. Akad. Nauk SSSR 307 (1989), no. 1, 49-53. translation in Soviet Math. Dokl. 40 (1990), no. 1, 43-48. MR.1017085

[Che90] I. V. Cherednik, Calculation of the monodromy of some $W$-invariant local systems of type $B, C$ and $D$, Funktsional. Anal. i Prilozhen. 24 (1990), no. 1, 88-89, DOI:10.1007/BF01077930 translation in Funct. Anal. Appl. 24 (1990), no. 1, 78-79. MR1052280

[DC13] K. De Commer, Representation theory of quantized enveloping algebras with interpolating real structure, SIGMA Symmetry Integrability Geom. Methods Appl. 9 (2013), Paper 081, 20, DOI:10.3842/SIGMA.2013.081. arXiv:1307.3642 [math.RT] MR3208147

[DCY15] K. De Commer and M. Yamashita, Tannaka-Kreı̆n duality for compact quantum homogeneous spaces II. Classification of quantum homogeneous spaces for quantum SU(2), J. Reine Angew. Math. 708 (2015), 143-171, DOI : 10.1515/crelle-2013-0074, arXiv:1212.3414 [math.0A] MR.3420332

[tDHO98] T. tom Dieck and R. Häring-Oldenburg, Quantum groups and cylinder braiding, Forum Math. 10 (1998), no. 5, 619-639, DOI: 10.1515/form.10.5.619 MR.1644317

[DM02] J. Donin and A. Mudrov, $\mathcal{U}_{q}(\mathrm{sl}(n))$-covariant quantization of symmetric coadjoint orbits via reflection equation algebra, Quantization, Poisson brackets and beyond (Manchester, 2001), Contemp. Math., vol. 315, Amer. Math. Soc., Providence, RI, 2002, pp. 61-79, DOI:10.1090/conm/315/05475 MR/1958831

[DKM03] J. Donin, P. P. Kulish, and A. I. Mudrov, On a universal solution to the reflection equation, Lett. Math. Phys. 63 (2003), no. 3, 179-194, DOI:10.1023/A:1024438101617, arXiv:math/0210242 [math.QA] MR 1992884

[Dri89] V. G. Drinfel'd, Quasi-Hopf algebras, Algebra i Analiz 1 (1989), no. 6, 114-148. Translation in Leningrad Math. J. 1 (1990), no. 6, 1419-1457. MR.1047964 (91b:17016)

[Enr07] B. Enriquez, Quasi-reflection algebras and cyclotomic associators, Selecta Math. (N.S.) 13 (2007), no. 3, 391463, DOI: 10.1007/s00029-007-0048-2, arXiv:math/0408035 [math.QA] MR2383601(2010e:17009)

[EE05] B. Enriquez and P. Etingof, Quantization of classical dynamical r-matrices with nonabelian base, Comm. Math. Phys. 254 (2005), no. 3, 603-650, DOI:10.1007/s00220-004-1243-z, arXiv:math/0311224 [math.QA] MR2126485 (2006a:17011)

[EGNO15] P. Etingof, S. Gelaki, D. Nikshych, and V. Ostrik. (2015). Tensor categories, Mathematical Surveys and Monographs, vol. 205, American Mathematical Society, Providence, RI, DOI:10.1090/surv/205, ISBN 978-1-47042024-6. MR 3242743

[GL00] V. A. Golubeva and V. Leksin, On a generalization of the Drinfeld-Kohno theorem, Proceedings of the Second ISAAC Congress, Vol. 2 (Fukuoka, 1999), 2000, pp. 1371-1386, Kluwer Acad. Publ., Dordrecht, DOI : 10.1007/978-1-4613-0271-1_61 MR.1940926 (2004a:32023)

[GS99] D. Gurevich and P. Saponov, Quantum sphere via reflection equation algebra. preprint (1999), available at arXiv:math/991141 [math.QA]

[Hel01] S. Helgason. (2001). Differential geometry, Lie groups, and symmetric spaces, Graduate Studies in Mathematics, vol. 34, American Mathematical Society, Providence, RI, DOI : 10.1090/gsm/034 ISBN 0-8218-2848-7. Corrected reprint of the 1978 original. MR 1834454

[Jan96] J. C. Jantzen. (1996). Lectures on quantum groups, Graduate Studies in Mathematics, vol. 6, American Mathematical Society, Providence, RI, ISBN 0-8218-0478-2. MR.1359532

[KW92] V. G. Kac and S. P. Wang, On automorphisms of Kac-Moody algebras and groups, Adv. Math. 92 (1992), no. 2, 129-195, DOI:10.1016/0001-8708(92) 90063-Q MR.1155464

[Kas95] C. Kassel. (1995). Quantum groups, Graduate Texts in Mathematics, vol. 155, Springer-Verlag, New York, ISBN 0-387-94370-6. MR.1321145 (96e:17041)

[KL94a] D. Kazhdan and G. Lusztig, Tensor structures arising from affine Lie algebras. III, J. Amer. Math. Soc. 7 (1994), no. 2, 335-381, DOI:10.2307/2152762 MR1239506 (94g:17048)

[KL94b] D. Kazhdan and G. Lusztig, Tensor structures arising from affine Lie algebras. IV, J. Amer. Math. Soc. 7 (1994), no. 2, 383-453, DOI:10.2307/2152763 MR1239507 (94g:17049)

[Kna02] A. W. Knapp. (2002). Lie groups beyond an introduction, 2nd ed., Progress in Mathematics, vol. 140, Birkhäuser Boston, Inc., Boston, MA, ISBN 0-8176-4259-5. MR.1920389

[KS09] S. Kolb and J. V. Stokman, Reflection equation algebras, coideal subalgebras, and their centres, Selecta Math. (N.S.) 15 (2009), no. 4, 621-664, DOI:10.1007/s00029-009-0007-1. MR2565052

[Kol14] S. Kolb, Quantum symmetric Kac-Moody pairs, Adv. Math. $267 \quad$ (2014), 395-469, DOI:10.1016/j.aim.2014.08.010, arXiv:1207.6036 [math.QA], MR3269184

[Kol17] S. Kolb, Braided module categories via quantum symmetric pairs. preprint (2017), available at arXiv: 1705.04238 [math.QA]

[KSS93] P. P. Kulish, R. Sasaki, and C. Schwiebert, Constant solutions of reflection equations and quantum groups, J. Math. Phys. 34 (1993), no. 1, 286-304, DOI:10.1063/1.530382 MR.1198638|(94e:82035)

[Lei94] A. Leibman, Some monodromy representations of generalized braid groups, Comm. Math. Phys. 164 (1994), no. 2, 293-304. MR1289327 (95k:52019)

[Let99] G. Letzter, Symmetric pairs for quantized enveloping algebras, J. Algebra 220 (1999), no. 2, 729-767, DOI: $10.1006 /$ jabr.1999.8015 MR.1717368

[Let00] G. Letzter, Harish-Chandra modules for quantum symmetric pairs, Represent. Theory 4 (2000), 64-96, DOI : 10.1090/S1088-4165-00-00087-X MR:1742961

[Let02] G. Letzter, Coideal subalgebras and quantum symmetric pairs, New directions in Hopf algebras, 2002, pp. 117165, Cambridge Univ. Press, Cambridge, arXiv:math/0103228 [math.QA] MR1913438 
[Let03] G. Letzter, Quantum symmetric pairs and their zonal spherical functions, Transform. Groups 8 (2003), no. 3, 261-292, DOI : 10.1007/s00031-003-0719-9 arXiv:math/0204103 [math.QA]. MR.1996417

[Lus93] G. Lusztig. (1993). Introduction to quantum groups, Progress in Mathematics, vol. 110, Birkhäuser Boston Inc., Boston, MA, ISBN 0-8176-3712-5. MR.1227098 (94m:17016)

[Mud07] A. Mudrov, Quantum conjugacy classes of simple matrix groups, Comm. Math. Phys. 272 (2007), no. 3, 635660, DOI: 10.1007/s00220-007-0222-6, arXiv:math/0412538 [math.QA] MR2304470

[Müg10] M. Müger, Tensor categories: a selective guided tour, Rev. Un. Mat. Argentina 51 (2010), no. 1, 95-163, arXiv:0804.3587 [math.CT] MR2681261 (2011f:18007)

[NT11a] S. Neshveyev and L. Tuset, Notes on the Kazhdan-Lusztig theorem on equivalence of the Drinfeld category and the category of $U_{q} \mathfrak{g}$-modules, Algebr. Represent. Theory 14 (2011), no. 5, 897-948, DOI : 10.1007/s10468-010-9223-9, arXiv:0711.4302 [math.QA], MR.2832264 (2012j:17022)

[NT11b] S. Neshveyev and L. Tuset, Symmetric invariant cocycles on the duals of q-deformations, Adv. Math. 227 (2011), no. 1, 146-169, DOI:10.1016/j.aim.2011.01.017, arXiv:0902.2365 [math.QA] MR:2782190

[NT12] S. Neshveyev and L. Tuset, Autoequivalences of the tensor category of $U_{q} \mathfrak{g}$-modules, Int. Math. Res. Not. IMRN 15 (2012), 3498-3508, arXiv:1012.4700 [math.QA] MR2959039

[NT13] S. Neshveyev and L. Tuset. (2013). Compact quantum groups and their representation categories, Cours Spécialisés [Specialized Courses], vol. 20, Société Mathématique de France, Paris, ISBN 978-2-85629-777-3. MR 3204665

[OV90] A. L. Onishchik and È. B. Vinberg. (1990). Lie groups and algebraic groups, Springer Series in Soviet Mathematics, Springer-Verlag, Berlin, DOI:10.1007/978-3-642-74334-4 ISBN 3-540-50614-4. Translated from the Russian and with a preface by D. A. Leites. MR.1064110

[Pod87] P. Podleś, Quantum spheres, Lett. Math. Phys. 14 (1987), no. 3, 193-202, DOI:10.1007/BF00416848 MR919322 (89b:46081)

[Was87] W. Wasow. (1987). Asymptotic expansions for ordinary differential equations, Dover Publications, Inc., New York, ISBN 0-486-65456-7. Reprint of the 1976 edition. MR919406

VRIJE Universiteit Brussel

Email address: kenny.de.commer@vub.be

UNIVERSITETET I OSLO

Email address: sergeyn@math.uio.no

OSLOMET - StORByuniversitetet

Email address: lars.tuset@hioa.no

OCHANOMIZU UNIVERSITY

Current address: Universitetet i Oslo

Email address: yamashita.makoto@ocha.ac.jp 